\title{
Asymptotic Properties of Recursive Particle Maximum Likelihood Estimation*
}

\author{
Vladislav Z. B. Tadić ${ }^{\dagger} \quad$ Arnaud Doucet ${ }^{\ddagger}$
}

\begin{abstract}
Using stochastic gradient search and the optimal filter derivative, it is possible to perform recursive maximum likelihood estimation in a non-linear state-space model. As the optimal filter and its derivative are analytically intractable for such a model, they need to be approximated numerically. In [25], a recursive maximum likelihood algorithm based on a particle approximation to the optimal filter derivative has been proposed and studied through numerical simulations. This algorithm and its asymptotic behavior are here analyzed theoretically. Under regularity conditions, we show that the algorithm accurately estimates maxima of the underlying (average) log-likelihood when the number of particles is sufficiently large. We also provide qualitative upper bounds on the estimation error in terms of the number of particles.
\end{abstract}

Keywords. Non-Linear State-Space Models, Recursive Maximum Likelihood Estimation, Sequential Monte Carlo Methods, System Identification.

AMS Subject Classification. Primary 62M09; Secondary 62L20, 65 C05.

\section{Introduction}

State-space models (also known as continuous-state hidden Markov models) are a class of stochastic processes capable of modeling complex time-series data and stochastic dynamical systems. These models can be viewed as a discrete-time Markov process which can be observed only through noisy measurements of its states.

In many applications, a state-space model depends on a parameter whose value needs to be estimated given a set of state-observations. Due to its practical and theoretical importance, parameter estimation in state-space and hidden Markov models has been extensively studied in the engineering and statistics literature; see e.g. [8], [14] and references cited therein. Among them, the methods based on maximum likelihood principle have gained much attention. Their asymptotic properties (convergence, convergence rate and asymptotic normality) have been analyzed thoroughly in a number of papers; see e.g. [4], [11], [12], [21], [23], [26], [27], [28]. Unfortunately, to the best of our knowledge, the existing results do not offer much information about recursive (i.e., online) maximum likelihood estimation in non-linear state-space models. However, in a number of different scenarios, the parameter indexing a state-space model needs to be estimated recursively. For example, this is much more computationally efficient for long observation sequences. In the maximum likelihood approach, this can be achieved using stochastic gradient search and the optimal filter derivative. Since the optimal filter and its derivative are not analytically tractable for a non-linear state-space model, they need to be approximated numerically. In [25], a recursive maximum likelihood algorithm based on a particle approximation to the optimal filter derivative has been proposed and it has been shown experimentally that the algorithm is stable and efficient. We show here that the algorithm proposed in [25] produces asymptotically accurate estimates of maxima to the underlying (average) log-likelihood. More specifically, we show that these estimates converge almost surely to a close

\footnotetext{
*A short version of the paper has been presented at the 2019 IEEE International Symposium on Information Theory.

${ }^{\dagger}$ School of Mathematics, University of Bristol, Bristol, United Kingdom (email: v.b.tadic@bristol.ac.uk).

${ }_{\ddagger}^{\ddagger}$ Department of Statistics, University of Oxford, Oxford, United Kingdom (email: doucet@stats.ox.ac.uk).
} 
vicinity of stationary points of the underlying log-likelihood. We also provide qualitative upper bounds on the radius of this vicinity. These bounds are expressed in terms of the number of particles used to approximate the filter and its derivative and directly characterize the (asymptotic) error of the recursive particle maximum likelihood algorithm proposed in [25]. The obtained results hold under strong mixing assumptions which are very commonly used in the particle filtering literature; see e.g. [8], [9], [10], [14]. To the best of our knowledge, the results presented here are the first to offer a rigorous analysis of recursive maximum likelihood estimation in non-linear state-space models.

The rest of this paper is organized as follows. In Section 2, non-linear state-space models and the corresponding recursive maximum likelihood algorithm are specified. In the same section, the main results of the paper are presented. In Section 3, a non-trivial example illustrating the main results is provided. The main results are proved in Sections $4-6$.

\section{Main Results}

\subsection{State-Space Models and Parameter Estimation}

To define state-space models, we use the following notation. For a set $\mathcal{Z}$ in a metric space, $\mathcal{B}(\mathcal{Z})$ denotes the collection of Borel subsets of $\mathcal{Z} . d_{x} \geq 1$ and $d_{y} \geq 1$ are integers, while $\mathcal{X} \in \mathcal{B}\left(\mathbb{R}^{d_{x}}\right)$ and $\mathcal{Y} \in \mathcal{B}\left(\mathbb{R}^{d_{y}}\right)$. $P\left(x, d x^{\prime}\right)$ is a transition kernel on $\mathcal{X}$, while $Q(x, d y)$ is a conditional probability measure on $\mathcal{Y}$ given $x \in \mathcal{X}$. $(\Omega, \mathcal{F}, P)$ is a probability space. A state-space model can be defined as the $\mathcal{X} \times \mathcal{Y}$-valued stochastic process $\left\{\left(X_{n}, Y_{n}\right)\right\}_{n \geq 0}$ on $(\Omega, \mathcal{F}, P)$ which satisfies

$$
P\left(\left(X_{n+1}, Y_{n+1}\right) \in B \mid X_{0: n}, Y_{0: n}\right)=\int I_{B}(x, y) Q(x, d y) P\left(X_{n}, d x\right)
$$

almost surely for each $B \in \mathcal{B}(\mathcal{X} \times \mathcal{Y}), n \geq 0 .\left\{X_{n}\right\}_{n \geq 0}$ are the unobservable model states, while $\left\{Y_{n}\right\}_{n \geq 0}$ are the observations. States $\left\{X_{n}\right\}_{n \geq 0}$ form a Markov chain, while $P\left(x, d x^{\prime}\right)$ is their transition kernel. The observations $\left\{Y_{n}\right\}_{n \geq 0}$ are mutually independent conditionally on $\left\{X_{n}\right\}_{n \geq 0}$, while $Q\left(X_{n}, d y\right)$ is the conditional distribution of $Y_{n}$ given $X_{0: n}$.

In this paper, we assume that the model $\left\{\left(X_{n}, Y_{n}\right)\right\}_{n \geq 0}$, can be accurately approximated by a parametric family of state-space models. To define such a family, we rely on the following notation. $d \geq 1$ is an integer, while $\Theta \subset \mathbb{R}^{d}$ is an open set. $\mathcal{P}(\mathcal{X})$ is the set of probability measures on $\mathcal{X} . \mu(d x)$ and $\nu(d y)$ are measures on $\mathcal{X}$ and $\mathcal{Y}$ (respectively). $p_{\theta}\left(x^{\prime} \mid x\right)$ and $q_{\theta}(y \mid x)$ are functions which map $\theta \in \Theta, x, x^{\prime} \in \mathcal{X}, y \in \mathcal{Y}$ to $[0, \infty)$ and satisfy

$$
\int_{\mathcal{X}} p_{\theta}\left(x^{\prime} \mid x\right) \mu\left(d x^{\prime}\right)=\int_{\mathcal{Y}} q_{\theta}(y \mid x) \nu(d y)=1
$$

for all $\theta \in \Theta, x \in \mathcal{X}$. A parametric family of state-space models can then be defined as a collection of $\mathcal{X} \times \mathcal{Y}$-valued stochastic processes $\left\{\left(X_{n}^{\theta, \lambda}, Y_{n}^{\theta, \lambda}\right)\right\}_{n>0}$ on $(\Omega, \mathcal{F}, P)$ which are parameterized by $\theta \in \Theta$, $\lambda \in \mathcal{P}(\mathcal{X})$ and satisfy

$$
\begin{aligned}
& P\left(\left(X_{0}^{\theta, \lambda}, Y_{0}^{\theta, \lambda}\right) \in B\right)=\iint I_{B}(x, y) q_{\theta}(y \mid x) \lambda(d x), \\
& P\left(\left(X_{n+1}^{\theta, \lambda}, Y_{n+1}^{\theta, \lambda}\right) \in B \mid X_{0: n}^{\theta, \lambda}, Y_{0: n}^{\theta, \lambda}\right)=\iint I_{B}(x, y) q_{\theta}(y \mid x) p_{\theta}\left(x \mid X_{n}^{\theta, \lambda}\right) \mu(d x) \nu(d y)
\end{aligned}
$$

almost surely for each $B \in \mathcal{B}(\mathcal{X} \times \mathcal{Y}), n \geq 0$.

We are interested in the identification of model parameters. This problem can be formulated as the estimation of the transition kernel $P\left(x, d x^{\prime}\right)$ and the conditional probability $Q(x, d y)$ given a realization of state-observations $\left\{Y_{n}\right\}_{n \geq 0}$. If the identification is based on the recursive maximum likelihood approach and the parametric model $\left\{\left(X_{n}^{\theta, \lambda}, Y_{n}^{\theta, \lambda}\right)\right\}_{n \geq 0}$, the estimation of $P\left(x, d x^{\prime}\right)$ and $Q(x, d y)$ reduces to the maximization of the log-likelihood rate associated with models $\left\{\left(X_{n}, Y_{n}\right)\right\}_{n \geq 0}$ and $\left\{\left(X_{n}^{\theta, \lambda}, Y_{n}^{\theta, \lambda}\right)\right\}_{n \geq 0}$. 
Here, $\left\{\left(X_{n}, Y_{n}\right)\right\}_{n \geq 0}$ is considered as the true system, while the parametric model $\left\{\left(X_{n}^{\theta, \lambda}, Y_{n}^{\theta, \lambda}\right)\right\}_{n \geq 0}$ is regarded as the candidate model.

To define the log-likelihood rate associated with models $\left\{\left(X_{n}, Y_{n}\right)\right\}_{n \geq 0}$ and $\left\{\left(X_{n}^{\theta, \lambda}, Y_{n}^{\theta, \lambda}\right)\right\}_{n \geq 0}$, we use the following notation: $q_{\theta}^{n}\left(y_{1: n} \mid \lambda\right)$ is the density of $Y_{1: n}^{\theta, \lambda}$, i.e.,

$$
q_{\theta}^{n}\left(y_{1: n} \mid \lambda\right)=\int \cdots \iint\left(\prod_{k=1}^{n}\left(q_{\theta}\left(y_{k} \mid x_{k}\right) p_{\theta}\left(x_{k} \mid x_{k-1}\right)\right)\right) \mu\left(d x_{n}\right) \cdots \mu\left(d x_{1}\right) \lambda\left(d x_{0}\right)
$$

for $\theta \in \Theta, \lambda \in \mathcal{P}(\mathcal{X}), y_{1: n}=\left(y_{1}, \ldots, y_{n}\right) \in \mathcal{Y}^{n}, n \geq 1 . l_{n}(\theta, \lambda)$ is the expected (average) log-likelihood of $Y_{1: n}$ given model $\left\{\left(X_{n}^{\theta, \lambda}, Y_{n}^{\theta, \lambda}\right)\right\}_{n \geq 0}$, i.e.,

$$
l_{n}(\theta, \lambda)=E\left(\frac{1}{n} \log q_{\theta}^{n}\left(Y_{1: n} \mid \lambda\right)\right) .
$$

Then, the log-likelihood rate for models $\left\{\left(X_{n}, Y_{n}\right)\right\}_{n \geq 0}$ and $\left\{\left(X_{n}^{\theta, \lambda}, Y_{n}^{\theta, \lambda}\right)\right\}_{n \geq 0}$ can be defined as the limit $\lim _{n \rightarrow \infty} l_{n}(\theta, \lambda)$. Under the assumptions adopted in this paper, $\lim _{n \rightarrow \infty} l_{n}(\theta, \lambda)$ exists and does not depend on $\lambda$ (see Lemmas 4.1 and 6.2). Throughout this paper, $l(\theta)$ denotes the log-likelihood rate for models $\left\{\left(X_{n}, Y_{n}\right)\right\}_{n \geq 0}$ and $\left\{\left(X_{n}^{\theta, \lambda}, Y_{n}^{\theta, \lambda}\right)\right\}_{n \geq 0}$, i.e.,

$$
l(\theta)=\lim _{n \rightarrow \infty} l_{n}(\theta, \lambda) .
$$

\subsection{Recursive Maximum Likelihood Algorithm}

Recursive maximum likelihood estimation in state-space models can be described as an online process maximizing the log-likelihood rate $l(\theta)$. As $l(\theta)$ and its gradient do not admit closed-form expressions for any non-linear state-space model, they need to be approximated numerically. We analyze here the recursive maximum likelihood algorithm proposed in [25]. In this algorithm, $\nabla l(\theta)$ is approximated by a particle method, while $l(\theta)$ is maximized by stochastic gradient search.

The recursive particle maximum likelihood algorithm proposed in [25, Sections 3.2, 2.2, Equations (26), (27), (18) - (22)] is defined by the following equations

$$
\begin{aligned}
W_{n+1, i}= & \frac{\sum_{j=1}^{N}\left(p_{\theta_{n}}\left(\hat{X}_{n+1, i} \mid \hat{X}_{n, j}\right) \nabla_{\theta} q_{\theta_{n}}\left(Y_{n} \mid \hat{X}_{n, j}\right)+\nabla_{\theta} p_{\theta_{n}}\left(\hat{X}_{n+1, i} \mid \hat{X}_{n, j}\right) q_{\theta_{n}}\left(Y_{n} \mid \hat{X}_{n, j}\right)\right)}{\sum_{j=1}^{N} p_{\theta_{n}}\left(\hat{X}_{n+1, i} \mid \hat{X}_{n, j}\right) q_{\theta_{n}}\left(Y_{n} \mid \hat{X}_{n, j}\right)} \\
& +\frac{\sum_{j=1}^{N} p_{\theta_{n}}\left(\hat{X}_{n+1, i} \mid \hat{X}_{n, j}\right) q_{\theta_{n}}\left(Y_{n} \mid \hat{X}_{n, j}\right) W_{n, j}}{\sum_{j=1}^{N} p_{\theta_{n}}\left(\hat{X}_{n+1, i} \mid \hat{X}_{n, j}\right) q_{\theta_{n}}\left(Y_{n} \mid \hat{X}_{n, j}\right)}, \\
\hat{W}_{n+1, i}= & W_{n+1, i}-\frac{1}{N} \sum_{j=1}^{N} W_{n+1, j}, \\
\theta_{n+1}= & \theta_{n}+\alpha_{n} \frac{\sum_{j=1}^{N}\left(q_{\theta_{n}}\left(Y_{n+1} \mid \hat{X}_{n+1, j}\right) \hat{W}_{n+1, j}+\nabla_{\theta} q_{\theta_{n}}\left(Y_{n+1} \mid \hat{X}_{n+1, j}\right)\right)}{\sum_{j=1}^{N} q_{\theta_{n}}\left(Y_{n+1} \mid \hat{X}_{n+1, j}\right)}
\end{aligned}
$$

for $n \geq 0,1 \leq i \leq N$. Here, $N \geq 1$ is an integer corresponding to the number of particles and $\left\{\alpha_{n}\right\}_{n \geq 0}$ is a sequence of positive real numbers. $\left\{\hat{X}_{n+1, i}: 1 \leq i \leq N\right\}$ are the particles generated through the sequential Monte Carlo scheme

$$
\hat{X}_{n+1, i} \sim \frac{\sum_{j=1}^{N} p_{\theta_{n}}\left(x \mid \hat{X}_{n, j}\right) q_{\theta_{n}}\left(Y_{n} \mid \hat{X}_{n, j}\right) \mu(d x)}{\sum_{j=1}^{N} q_{\theta_{n}}\left(Y_{n} \mid \hat{X}_{n, j}\right)} .
$$

In (4), $\left\{\hat{X}_{n+1, i}: 1 \leq i \leq N\right\}$ are sampled independently one from another and independently from $\left\{X_{k}\right.$ : $0 \leq k \leq n\},\left\{\theta_{k}, Y_{k}, \hat{X}_{k, i}: 0 \leq k<n, 1 \leq i \leq N\right\}$. Moreover, in (1)-(4), $\theta_{0} \in \Theta,\left\{\hat{X}_{0, i}: 1 \leq i \leq N\right\} \subset \mathcal{X}$ and $\left\{W_{0, i}: 1 \leq i \leq N\right\} \subset \mathbb{R}^{d \times N}$ are selected independently from $\left(X_{0}, Y_{0}\right)$. 
Remark. Recursion (3) usually involves a device which keeps $\left\{\theta_{n}\right\}_{n \geq 0}$ within a compact subset of $\Theta$. This device is usually based on the projection to a compact domain (for details, see [6, Section 5.4], [18, Sections 5.1, 5.2] and references cited therein). As algorithm (1) - (4) is already a very complex procedure, this aspect is not considered here. Instead, similarly as in [3, Part II], [6], [18], [28], our results on the asymptotic behavior of the algorithm (Theorem 2.1, below) are expressed in a local form.

The variables appearing in algorithm (1) - (4) have the following meaning. $\theta_{0}, \hat{X}_{0,1}, \ldots, \hat{X}_{0, N}$ and $W_{0,1}, \ldots, W_{0, N}$ are the initial conditions. $\hat{X}_{n, 1}, \ldots, \hat{X}_{n, N}$ are particles whose empirical distribution approximates the predictive distribution of $X_{n}$ given $Y_{1}, \ldots, Y_{n-1}$ and its derivative (computed using parameter $\theta_{n}$ at time $n$ ), while $W_{n, 1}, \ldots, W_{n, N}$ are vector-valued weights in the particle approximation to this derivative. ${ }^{1} \theta_{n}$ is an estimate of maxima to the log-likelihood rate $l(\theta) . \alpha_{n}$ is the step-size in recursion (1). Recursion (3) is a stochastic gradient search maximizing $l(\theta) .{ }^{2}$ Recursions (1) and (4) are procedures through which the particle approximations to the optimal filter and its derivative are updated. More details on the recursive particle maximum likelihood algorithm can be found in [25].

\subsection{Convergence Results}

To formulate the assumptions under which the analysis of the recursive particle maximum likelihood estimation procedure is carried out, we introduce further notation. $\mathbb{N}_{0}$ is the set of non-negative integers, while $\mathbb{C}^{d}$ is the set $d$-dimensional complex valued vectors. For $\boldsymbol{\alpha}=\left(a_{1}, \ldots, a_{d}\right) \in \mathbb{N}_{0}^{d}, \theta=\left(t_{1}, \ldots, t_{d}\right) \in \mathbb{R}^{d}$, notation $|\boldsymbol{\alpha}|$ and $\partial_{\theta}^{\alpha}$ stand for

$$
|\boldsymbol{\alpha}|=a_{1}+\cdots+a_{d}, \quad \partial_{\theta}^{\boldsymbol{\alpha}}=\frac{\partial_{\theta}^{|\boldsymbol{\alpha}|}}{\partial t_{1}^{a_{1}} \cdots t_{d}^{a_{d}}} .
$$

For $\eta \in \mathbb{C}^{d}$, let $\|\eta\|$ denote the Euclidean norm of $\eta$. For $\delta \in(0, \infty), \eta \in \mathbb{C}^{d}$ and $A \subset \mathbb{C}^{d}$, let $V_{\delta}(A)$ and $d(\eta, A)$ denote the $\delta$-vicinity of $A$ and the distance between $\eta$, $A$, i.e.,

$$
d(\eta, A)=\inf _{\eta^{\prime} \in A}\left\|\eta-\eta^{\prime}\right\|, \quad V_{\delta}(A)=\left\{\eta^{\prime} \in \mathbb{C}^{d}: \exists \eta^{\prime \prime} \in A,\left\|\eta^{\prime}-\eta^{\prime \prime}\right\|<\delta\right\} .
$$

Let $Q$ be any compact set satisfying $Q \subset \Theta$. The asymptotic properties of algorithm (1) - (4) are analyzed under the following assumptions.

Assumption 2.1. $\sum_{n=0}^{\infty} \alpha_{n}=\infty, \sum_{n=0}^{\infty} \alpha_{n}^{2}<\infty$ and $\sum_{n=0}^{\infty}\left|\alpha_{n}-\alpha_{n+1}\right|<\infty$.

Assumption 2.2. There exist a probability measure $\pi(d x)$ and real numbers $\rho \in(0,1), K \in[1, \infty)$ such that

$$
\left|P^{n}(x, B)-\pi(B)\right| \leq K \rho^{n}
$$

for all $x \in \mathcal{X}, B \in \mathcal{B}(\mathcal{X}), n \geq 0$.

Assumption 2.3. There exists a real number $\varepsilon_{Q} \in(0,1)$ such that

$$
\varepsilon_{Q} \leq p_{\theta}\left(x^{\prime} \mid x\right) \leq \frac{1}{\varepsilon_{Q}}, \quad \varepsilon_{Q} \leq q_{\theta}(y \mid x) \leq \frac{1}{\varepsilon_{Q}}
$$

for all $\theta \in Q, x, x^{\prime} \in \mathcal{X}, y \in \mathcal{Y}$.

${ }^{1}$ The empirical measures

$$
\frac{1}{N} \sum_{i=1}^{N} \delta_{\hat{X}_{n, i}}(d x), \quad \frac{1}{N} \sum_{i=1}^{N}\left(W_{n, i}-\frac{1}{N} \sum_{j=1}^{N} W_{n, j}\right) \delta_{\hat{X}_{n, i}}(d x)
$$

can be viewed as particle approximations (respectively) to the optimal (one-step) predictor and its gradient at discrete-time $n$. Here and throughout the paper, $\delta_{x}\left(d x^{\prime}\right)$ denotes the Dirac measure centered at $x \in \mathcal{X}$.

${ }^{2}$ The fraction on the right-hand side of (3) is a Monte Carlo estimate of $l(\theta)$. 
Assumption 2.4. There exists a real number $K_{1, Q} \in[1, \infty)$ such that

$$
\begin{aligned}
& \max \left\{\left\|\nabla_{\theta} p_{\theta}\left(x^{\prime} \mid x\right)\right\|,\left\|\nabla_{\theta} q_{\theta}(y \mid x)\right\|\right\} \leq K_{1, Q}, \\
& \max \left\{\left|p_{\theta}\left(x^{\prime} \mid x\right)-p_{\theta^{\prime}}\left(x^{\prime} \mid x\right)\right|,\left|q_{\theta}(y \mid x)-q_{\theta^{\prime}}(y \mid x)\right|\right\} \leq K_{1, Q}\left\|\theta-\theta^{\prime}\right\|, \\
& \max \left\{\left\|\nabla_{\theta} p_{\theta}\left(x^{\prime} \mid x\right)-\nabla_{\theta} p_{\theta^{\prime}}\left(x^{\prime} \mid x\right)\right\|,\left\|\nabla_{\theta} q_{\theta}(y \mid x)-\nabla_{\theta} q_{\theta^{\prime}}(y \mid x)\right\|\right\} \leq K_{1, Q}\left\|\theta-\theta^{\prime}\right\|
\end{aligned}
$$

for all $\theta, \theta^{\prime} \in Q, x, x^{\prime} \in \mathcal{X}, y \in \mathcal{Y}$.

Assumption 2.5. $p_{\theta}\left(x^{\prime} \mid x\right)$ and $q_{\theta}(y \mid x)$ are $p$-times differentiable in $\theta$ for each $\theta \in \Theta, x, x^{\prime} \in \mathcal{X}, y \in \mathcal{Y}$, where $p>d$. Moreover, there exists a real number $K_{2, Q} \in[1, \infty)$ such that

$$
\left|\partial_{\theta}^{\boldsymbol{\alpha}} p_{\theta}\left(x^{\prime} \mid x\right)\right| \leq K_{2, Q}, \quad\left|\partial_{\theta}^{\boldsymbol{\alpha}} q_{\theta}(y \mid x)\right| \leq K_{2, Q}
$$

for all $\theta \in Q, x, x^{\prime} \in \mathcal{X}, y \in \mathcal{Y}, \boldsymbol{\alpha} \in \mathbb{N}_{0}^{d}$ satisfying $|\boldsymbol{\alpha}| \leq p$.

Assumption 2.6. $p_{\theta}\left(x^{\prime} \mid x\right)$ and $q_{\theta}(y \mid x)$ are real-analytic in $\theta$ for each $\theta \in \Theta, x, x^{\prime} \in \mathcal{X}, y \in \mathcal{Y}$. Moreover, $p_{\theta}\left(x^{\prime} \mid x\right)$ and $q_{\theta}(y \mid x)$ have (complex-valued) continuations $\hat{p}_{\eta}\left(x^{\prime} \mid x\right)$ and $\hat{q}_{\eta}(y \mid x)$ with the following properties:

(i) $\hat{p}_{\eta}\left(x^{\prime} \mid x\right)$ and $\hat{q}_{\eta}(y \mid x)$ map $\eta \in \mathbb{C}^{d}, x, x^{\prime} \in \mathcal{X}, y \in \mathcal{Y}$ to $\mathbb{C}$

(ii) $\hat{p}_{\theta}\left(x^{\prime} \mid x\right)=p_{\theta}\left(x^{\prime} \mid x\right)$ and $\hat{q}_{\theta}(y \mid x)=q_{\theta}(y \mid x)$ for all $\theta \in \Theta, x, x^{\prime} \in \mathcal{X}, y \in \mathcal{Y}$.

(iii) There exists a real number $\delta_{Q} \in(0,1)$ such that $\hat{p}_{\eta}\left(x^{\prime} \mid x\right)$ and $\hat{q}_{\eta}(y \mid x)$ are analytic in $\eta$ for each $\eta \in V_{\delta_{Q}}(Q), x, x^{\prime} \in \mathcal{X}, y \in \mathcal{Y}$.

(iv) There exists a real number $K_{3, Q} \in(0,1)$ such that

$$
\left|\hat{p}_{\eta}\left(x^{\prime} \mid x\right)\right| \leq K_{3, Q}, \quad\left|\hat{q}_{\eta}(y \mid x)\right| \leq K_{3, Q}
$$

for all $\eta \in V_{\delta_{Q}}(Q), x, x^{\prime} \in \mathcal{X}, y \in \mathcal{Y}$.

Assumption 2.1 corresponds to the step-size sequence $\left\{\alpha_{n}\right\}_{n \geq 0}$ and its asymptotic properties. This assumption is standard in any asymptotic analysis of stochastic gradient search and stochastic approximation (see e.g., [3], [6], [18]). It holds when $\alpha_{n}=1 / n^{a}$ for $n \geq 1$, where $a \in(1 / 2,1]$.

Assumption 2.2 is related to the stability of the true system $\left\{\left(X_{n}, Y_{n}\right)\right\}_{n \geq 0}$. It requires $\left\{X_{n}\right\}_{n \geq 0}$ to be uniformly ergodic. Assumption 2.3 implies the stability of the optimal filter for $\left\{\left(X_{n}^{\theta, \lambda}, Y_{n}^{\theta, \lambda}\right)\right\}_{n \geq 0}$; i.e. it ensures that the optimal filter forgets its initial condition exponentially fast. Assumption 2.2 and 2.3 are restrictive from the theoretical point of view as they implicitly require the state and observation spaces $\mathcal{X}$ and $\mathcal{Y}$ to be bounded. However, these assumptions are very commonly used in the literature; see, e.g., [8], $[9],[14]$.

Assumptions $2.4-2.6$ are related to the parameterization of the candidate models $\left\{\left(X_{n}^{\theta, \lambda}, Y_{n}^{\theta, \lambda}\right)\right\}_{n \geq 0}$ and its analytical properties; i.e. the analytical properties of conditional densities $p_{\theta}\left(x^{\prime} \mid x\right)$ and $q_{\theta}(y \mid x)$. The purpose of Assumption 2.4 is to ensure that the Poisson equation associated with algorithm (1) - (4) has a locally Lipschitz solution (see Lemma 5.4). Assumption 2.4 also ensures that the log-likelihood rate $l(\theta)$ is Lipschitz continuously differentiable (see Lemmas 4.1,6.2). This Poisson equation plays a crucial role in the analysis of the asymptotic error in the Monte Carlo estimation of $\nabla l(\theta)$ (see Lemma 6.1 and (19)), while the Lipschitz continuity of $\nabla l(\theta)$ allows us to analyze algorithm (1) - (4) using the results on Lipschitz gradient flows (see Theorem 2.1, Part (i)). The purpose of Assumption 2.5 is to provide for $l(\theta)$ to be at least $(d+1)$-times differentiable (see Lemma 6.2, Part (ii)), while Assumption 2.6 ensures for $l(\theta)$ to be real-analytic (see Lemma 6.2, Part (iii)). These analytical properties of $l(\theta)$ allows us to establish qualitative upper bounds on the asymptotic error in the estimation of maxima to $l(\theta)$ (see Theorem 2.1, Parts (ii), (iii)).

In order to state the main results of the paper, we need further notation. $\mathcal{S}$ and $l(\mathcal{S})$ are the sets of stationary points and critical values of $l(\theta)$ (respectively), i.e.,

$$
\mathcal{S}=\{\theta \in \Theta: \nabla l(\theta)=0\}, \quad l(\mathcal{S})=\{l(\theta): \theta \in \mathcal{S}\} .
$$

$\pi: \mathbb{R} \times \Theta \rightarrow \Theta$ is the solution to the ODE $d \theta / d t=\nabla l(\theta)$ which satisfies the initial condition $\pi(0, \theta)=\theta$ for $\theta \in \Theta$. $\mathcal{R}$ is the set of chain-recurrent points of the ODE $d \theta / d t=\nabla l(\theta)$, i.e., $\theta \in \mathcal{R}$ if and only if for 
any $\delta, t \in(0, \infty)$, there exist an integer $n \geq 1$, real numbers $t_{1}, \ldots, t_{n} \in[t, \infty)$ and vectors $\vartheta_{1}, \ldots, \vartheta_{n} \in \Theta$ (each of which can depend on $\theta, \delta, t$ ) such that

$$
\left\|\vartheta_{1}-\theta\right\| \leq \delta, \quad\left\|\pi\left(t_{n}, \vartheta_{n}\right)-\theta\right\| \leq \delta, \quad\left\|\vartheta_{k+1}-\pi\left(t_{k}, \vartheta_{k}\right)\right\| \leq \delta
$$

for $1 \leq k<n$.

Remark. Chain-recurrent points $\mathcal{R}$ can be interpreted as limit points of slightly perturbed solutions to the $O D E d \theta / d t=\nabla l(\theta)$. Since the piecewise linear interpolation of sequence $\left\{\theta_{n}\right\}_{n \geq 0}$ is such a solution (see (19) and Lemma 6.1; see also [30, Section 5]), the chain-recurrence is closely related to the asymptotic behavior of algorithm (1) - (4). Regarding stationary and chain-recurrent points, the following relationship can be established. If $l(\theta)$ is Lipschitz continuously differentiable, then all stationary points $\mathcal{S}$ are chain-recurrent for the $O D E d \theta / d t=\nabla l(\theta)$ (i.e., $\mathcal{S} \subseteq \mathcal{R}$ ). If additionally $l(\mathcal{S})$ is of a zero Lebesgue measure (which holds when $l(\theta)$ is d-times continuously differentiable), then all chain-recurrent points $\mathcal{R}$ are stationary for the $O D E d \theta / d t=\nabla l(\theta)$ (i.e., $\mathcal{S}=\mathcal{R}$ ). However, if $l(\theta)$ is only Lipschitz continuously differentiable, then $\mathcal{S}=\mathcal{R}$ does not necessarily hold and $\mathcal{R} \backslash \mathcal{S} \neq \emptyset$ is quite possible (for details, see [16, Section 4]). For more details on chain-recurrence, see [1], [2], [6].

Let $Q$ be any compact set satisfying $Q \subset \Theta$, while $\Lambda_{Q}$ is the event defined by

$$
\Lambda_{Q}=\liminf _{n \rightarrow \infty}\left\{\theta_{n} \in Q\right\}=\bigcup_{n=0}^{\infty} \bigcap_{k=n}^{\infty}\left\{\theta_{k} \in Q\right\}
$$

Then, the main results of this paper are summarized in the next theorem.

Theorem 2.1. (i) If Assumptions 2.1 - 2.4 hold, then there exists a non-decreasing function $\psi_{Q}:[0, \infty) \rightarrow$ $[0, \infty)$ depending only on $\left.l(\theta), p_{\theta}\left(x^{\prime} \mid x\right), q_{\theta}(y \mid x)\right)$ such that $\lim _{t \rightarrow 0} \psi_{Q}(t)=\psi_{Q}(0)=0$ and

$$
\limsup _{n \rightarrow \infty} d\left(\theta_{n}, \mathcal{R}\right) \leq \psi_{Q}\left(\frac{1}{N}\right)
$$

almost surely on $\Lambda_{Q}$.

(ii) If Assumptions 2.1 - 2.5 hold, then there exists a real number $L_{1, Q} \in[1, \infty)$ (independent of $N$ and depending only on $\left.l(\theta), p_{\theta}\left(x^{\prime} \mid x\right), q_{\theta}(y \mid x)\right)$ such that

$$
\limsup _{n \rightarrow \infty}\left\|\nabla l\left(\theta_{n}\right)\right\| \leq \frac{L_{1, Q}}{N^{q / 2}}, \quad \limsup _{n \rightarrow \infty} l\left(\theta_{n}\right)-\liminf _{n \rightarrow \infty} l\left(\theta_{n}\right) \leq \frac{L_{1, Q}}{N^{q}}
$$

almost surely on $\Lambda_{Q}$, where $q=(p-d) /(p-1)$.

(iii) If Assumptions 2.1 - 2.4 and 2.6 hold, then there exist real numbers $r_{Q} \in(0,1), L_{2, Q} \in[1, \infty)$ (independent of $N$ and depending only on $\left.l(\theta), p_{\theta}\left(x^{\prime} \mid x\right), q_{\theta}(y \mid x)\right)$ such that

$$
\limsup _{n \rightarrow \infty} d\left(\theta_{n}, \mathcal{S}\right) \leq \frac{L_{2, Q}}{N^{r Q}}, \quad \limsup _{n \rightarrow \infty}\left\|\nabla l\left(\theta_{n}\right)\right\| \leq \frac{L_{2, Q}}{N^{1 / 2}}, \quad \limsup _{n \rightarrow \infty} d\left(l\left(\theta_{n}\right), l(\mathcal{S})\right) \leq \frac{L_{2, Q}}{N}
$$

almost surely on $\Lambda_{Q}$.

Theorem 2.1 is proved in Section 6 .

Remark. The function $\psi_{Q}(t)$ and the real numbers $L_{1, Q}, L_{2, Q}$ depend on $p_{\theta}\left(x^{\prime} \mid x\right), q_{\theta}(y \mid x)$ through constants $\varepsilon_{Q}, K_{1, Q}$ (specified in Assumptions 2.3, 2.4). $\psi_{Q}(t)$ also depends on $l(\theta)$ through a Lipschitz constant of $\nabla l(\theta)$, an upper bound of $\|\nabla l(\theta)\|$ and the geometric properties of $\mathcal{R}$. $L_{1, Q}, L_{2, Q}$ depend on $l(\theta)$ through a Lipschitz constant of $\nabla l(\theta)$ and an upper bound of $\|\nabla l(\theta)\|$. Additionally, $L_{1, Q}, L_{2, Q}$ also 
depend on $l(\theta)$ through the Yomdin and Lojasiewicz constants for $l(\theta) .{ }^{3} r_{Q}$ is the Lojasiewicz exponent for $l(\theta)$. For further details on how $\psi_{Q}(t), r_{Q}, L_{1, Q}, L_{2, Q}$ depend on $l(\theta), p_{\theta}\left(x^{\prime} \mid x\right), q_{\theta}(y \mid x)$, see [30].

As algorithm (1) - (4) is a stochastic gradient search maximizing the average log-likelihood $l(\theta)$, the asymptotic properties of sequences $\left\{\theta_{n}\right\}_{n \geq 0},\left\{l\left(\theta_{n}\right)\right\}_{n \geq 0}$ and $\left\{\nabla l\left(\theta_{n}\right)\right\}_{n \geq 0}$ provide a natural way to characterize the asymptotic behavior of this algorithm. If the estimation of $\nabla l(\theta)$ in algorithm (1) - (4) were based on the exact optimal filter instead of a particle approximation, the corresponding estimator would be asymptotically consistent. Then, according to the existing results on stochastic optimization, sequences $\left\{\theta_{n}\right\}_{n \geq 0},\left\{l\left(\theta_{n}\right)\right\}_{n \geq 0}$ and $\left\{\nabla l\left(\theta_{n}\right)\right\}_{n \geq 0}$ would exhibit the following behavior. If $\nabla l(\theta)$ was estimated using the exact optimal filter and $l(\theta)$ was Lipschitz continuously differentiable, then the limits

$$
\lim _{n \rightarrow \infty} d\left(\theta_{n}, \mathcal{R}\right)=0, \quad \lim _{n \rightarrow \infty} d\left(l\left(\theta_{n}\right), l(\mathcal{R})\right)=0, \quad \liminf _{n \rightarrow \infty} d\left(\theta_{n}, \mathcal{S}\right)=0
$$

would hold almost surely on the event $\left\{\sup _{n \geq 0}\left\|\theta_{n}\right\|<\infty, \inf _{n \geq 0} d\left(\theta_{n}, \Theta^{c}\right)>0\right\}$; see, e.g., [1, Proposition 4.1, Theorem 5.7]. If $l(\theta)$ was additionally $(d+1)$-times differentiable, then the limits

$$
\lim _{n \rightarrow \infty} d\left(\theta_{n}, \mathcal{S}\right)=0, \quad \lim _{n \rightarrow \infty} \nabla l\left(\theta_{n}\right)=0, \quad \lim _{n \rightarrow \infty} d\left(l\left(\theta_{n}\right), l(\mathcal{S})\right)=0, \quad \limsup _{n \rightarrow \infty} l\left(\theta_{n}\right)=\liminf _{n \rightarrow \infty} l\left(\theta_{n}\right)
$$

would hold almost surely on $\left\{\sup _{n \geq 0}\left\|\theta_{n}\right\|<\infty, \inf _{n \geq 0} d\left(\theta_{n}, \Theta^{c}\right)>0\right\}$ (see e.g., [1, Corollary 6.7]). Since algorithm (1) - (4) estimates $\nabla l(\theta)$ using a particle approximation, the corresponding estimator is biased. Consequently, the limits (10), (11) do not hold for algorithm (1) - (4). Instead, the following limits

$$
\limsup _{n \rightarrow \infty} d\left(\theta_{n}, \mathcal{R}\right), \quad \limsup _{n \rightarrow \infty}\left\|\nabla l\left(\theta_{n}\right)\right\|, \quad \limsup _{n \rightarrow \infty} d\left(l\left(\theta_{n}\right), l(\mathcal{R})\right), \quad \limsup _{n \rightarrow \infty} l\left(\theta_{n}\right)-\liminf _{n \rightarrow \infty} l\left(\theta_{n}\right)
$$

take strictly positive values. These limits directly depend on the accuracy of the particle approximations to the optimal filter and its derivative.

Theorem 2.1 provides qualitative upper bounds on the limits (12) in terms of the number of particles $N$ and the analytical properties of the log-likelihood rate $l(\theta)$. These bounds are of the almost sure type and based on the strong mixing condition (Assumption 2.3). As such, they can be considered as of the worstcase type. Moreover, these bounds can be rather loose in scenarios for which the strong mixing condition is too conservative or even undesirable (e.g., when $x \mapsto q_{\theta}(y \mid x)$ is concentrated while $x^{\prime} \mapsto p_{\theta}\left(x^{\prime} \mid x\right)$ is diffuse). This is partly due to the fact that we have analyzed the bootstrap particle filter (4) which is well-known to perform poorly in these scenarios. By using more sophisticated schemes which sample particles using a distribution dependent on the observations [24], we believe that the bounds could be improved. These bounds could also be made tighter using the (non-mixing) assumptions on the optimal filter stability adopted in [13]. However, this would require substantial generalization of the existing results on the stability of the optimal filter derivatives and their particle approximations (i.e., of the results of [29], [33]). Since the analysis of the optimal filter derivatives and their particle approximations would be very difficult under non-mixing stability conditions and since the result presented here are already complex, this generalization is left for future research.

\footnotetext{
${ }^{3}$ If $l(\theta)$ is $p$-times differentiable and $p>d$ (which is true under Assumptions 2.2 - 2.5; see Part (ii) of Lemma 6.2), there exists a real number $M_{1, Q} \in[1, \infty)$ such that

$$
m(\{l(\theta): \theta \in Q,\|\nabla l(\theta)\| \leq \varepsilon\}) \leq M_{1, Q} \varepsilon^{q}
$$

for all $\varepsilon \in[1, \infty)$, where $m(\cdot)$ is the Lebesgue measure on $\mathbb{R}^{d}$ ( $q$ is specified in the statement of Theorem 2.1). This result is known as the Yomdin theorem (see [35, Theorem 1.2]), while $M_{1, Q}$ is referred to as the Yomdin exponent.

If $l(\theta)$ is real-analytic on $\Theta$ (which holds under Assumptions 2.2-2.4, 2.6; see Part (iii) of Lemma 6.2), there exist real numbers $r_{Q} \in(0,1), M_{2, Q}, M_{3, Q} \in[1, \infty)$ such that

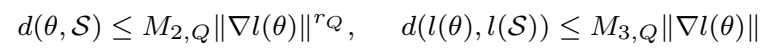

for all $\theta \in Q$. These inequalities are known as the Lojasiewicz inequalities (see [5, Theorem 6.4, Remark 6.5], [17, Theorem ŁI, Page 775]). $M_{2, Q}, M_{3, Q}$ are referred to as the Lojasiewicz constants, while $r_{Q}$ is called the Lojasiewicz exponent.
} 


\subsection{Outline of Proofs of Convergence Results}

An outline/summary of the main steps and key ideas in the proof of Theorem 2.1 is provided here. These steps and ideas can be described as follows:

Step 1: Algorithm (1) - (4) is transformed to stochastic approximation with Markovian dynamics. More specifically, it is rewritten as

$$
\begin{aligned}
W_{n+1} & =W_{n} A_{\theta_{n}}\left(V_{n}, V_{n+1}\right)+B_{\theta_{n}}\left(V_{n}, V_{n+1}\right), \\
\theta_{n+1} & =\theta_{n}+\alpha_{n}\left(W_{n+1} C_{\theta_{n}}\left(V_{n+1}\right)+D_{\theta_{n}}\left(V_{n+1}\right)\right) .
\end{aligned}
$$

The same algorithm is also rewritten as

$$
\theta_{n+1}=\theta_{n}+\alpha_{n} H\left(\theta_{n}, Z_{n+1}\right)
$$

Here, $\left\{V_{n}\right\}_{n \geq 0},\left\{W_{n}\right\}_{n \geq 0}$ and $\left\{Z_{n}\right\}_{n \geq 0}$ are stochastic processes

$$
V_{n}=\left(Y_{n}, X_{n}, \hat{X}_{n}\right), \quad W_{n}=\left(W_{n, 1}, \ldots, W_{n, N}\right), \quad Z_{n}=\left(V_{n}, W_{n}\right),
$$

while $\hat{X}_{n}$ is the vector of particles $\hat{X}_{n}=\left(\hat{X}_{n, 1}, \ldots, \hat{X}_{n, N}\right)\left(W_{n}\right.$ is the $d \times N$ matrix whose $j$-th column is $\left.W_{n, j}\right) . A_{\theta}\left(v, v^{\prime}\right), B_{\theta}\left(v, v^{\prime}\right), C_{\theta}(v), D_{\theta}(v)$ and $H(\theta, z)$ are suitably chosen functions which are defined precisely in (27), (28), (29). Equations (13) - (15) are a compact form of (1) - (3), while terms

$$
W_{n+1} C_{\theta_{n}}\left(V_{n+1}\right)+D_{\theta_{n}}\left(V_{n+1}\right), \quad H\left(\theta_{n}, Z_{n+1}\right)
$$

can be viewed as Monte Carlo estimators of $\nabla l\left(\theta_{n}\right)$. Aggregate process $\left\{\left(\theta_{n}, Z_{n}\right)\right\}_{n \geq 0}$ is a Markov chain, while stochastic processes $\left\{V_{n}\right\}_{n \geq 0}$ and $\left\{Z_{n}\right\}_{n \geq 0}$ can be interpreted as Markov chains controlled by estimates $\left\{\theta_{n}\right\}_{n \geq 0}($ see $(34),(35))$.

Step 2: We analyze conditional probability measure of $V_{n+1}$ given $V_{n}=v, \theta_{n}=\theta$, which is denoted by $T_{\theta}\left(v, d v^{\prime}\right)$ and precisely defined in (32). It is shown that $T_{\theta}\left(v, d v^{\prime}\right)$ is geometrically ergodic with a rate (locally) uniform in $\theta$. It is also established that $T_{\theta}\left(v, d v^{\prime}\right)$ is (locally) Lipschitz continuous in $\theta$. The details are included in Lemma 5.1.

Step 3: We consider the conditional expectations of the products

$$
A_{\theta_{0}}\left(V_{0}, V_{1}\right) \cdots A_{\theta_{n-1}}\left(V_{n-1}, V_{n}\right) C_{\theta_{n}}\left(V_{n}\right), \quad B_{\theta_{0}}\left(V_{0}, V_{1}\right) A_{\theta_{1}}\left(V_{1}, V_{2}\right) \cdots A_{\theta_{n-1}}\left(V_{n-1}, V_{n}\right) C_{\theta_{n}}\left(V_{n}\right)
$$

given $\theta_{0}=\theta, \ldots, \theta_{n}=\theta, V_{0}=v$. These conditional expectations are denoted by $\Phi_{\theta}^{n}(v), \Psi_{\theta}^{n}(v)$ and defined precisely in (40), (41). Using results on stochastic matrices (see Appendix 1) and the results of Step 2, it is shown that functions $\Phi_{\theta}^{n}(v), \Psi_{\theta}^{n}(v)$ converge exponentially to zero as $n \rightarrow \infty$ with rates (locally) uniform in $\theta$. The same functions are also shown to be (locally) Lipschitz continuous with Lipschitz constants tending exponentially to zero as $n \rightarrow \infty$. The details are provided in Lemma 5.3.

Step 4: Function $\left(\Pi^{n} H\right)(\theta, z)$ and its properties are analyzed, where $\left(\Pi^{n} H\right)(\theta, z)$ is the conditional expectation of $H\left(\theta_{n}, Z_{n+1}\right)$ given $\theta_{0}=\theta, \ldots, \theta_{n}=\theta, Z_{0}=z$ (see $(78)$ ). $\Pi_{\theta}\left(z, d z^{\prime}\right)$ is the conditional probability measure of $Z_{n+1}$ given $Z_{n}=z, \theta_{n}=\theta$, which is defined precisely in (33). Relying on the results of Step 3, it is shown that there exists a function $h(\theta)$ such that $\left(\Pi^{n} H\right)(\theta, z)$ converges exponentially to $h(\theta)$ as $n \rightarrow \infty$ at a rate (locally) uniform in $\theta$. It is also shown that $\left(\Pi^{n} H\right)(\theta, z)-h(\theta)$ is (locally) Lipschitz continuous in $\theta$ with a Lipschitz constant tending exponentially to zero as $n \rightarrow \infty$. The details are included in Lemma 5.4 (see (43), (83), (88)).

Step 5: The Poisson equation associated with algorithm (1) - (4) (i.e., with functions $H(\theta, z), h(\theta)$ and the transition kernel $\left.\Pi_{\theta}\left(z, d z^{\prime}\right)\right)$ and its properties are considered. Relying on the results of Step 4, it is shown that the Poisson equation has a solution and that the solution is (locally) Lipschitz continuous in $\theta$. The details are provided in Lemma 5.4.

Step 6: The weight sequence $\left\{W_{n}\right\}_{n \geq 0}$ and its stability are studied. Using results on stochastic matrices (see Appendix 1), it is shown that $W_{n}$ is (deterministically) bounded in $n$. The details are contained in Lemma 5.5. 
Step 7: The Monte Carlo estimators (17) and their statistical properties are analyzed. By exploiting the results of [33], it is shown that the asymptotic bias of these estimators are inversely proportional to $N$ with a multiplicative constant uniform in $\theta$. The details are included in Lemma 5.6.

Step 8: Algorithm (1) - (4) is transformed to a stochastic gradient search with additive noise. More specifically, it is rewritten as

$$
\theta_{n+1}=\theta_{n}+\alpha_{n}\left(\nabla l\left(\theta_{n}\right)+\xi_{n}\right)
$$

Moreover, the additive noise $\xi_{n}$ is decomposed as $\xi_{n}=\zeta_{n}+\eta_{n}$, where $\xi_{n}=H\left(\theta_{n}, Z_{n+1}\right)-h\left(\theta_{n}\right), \zeta_{n}=$ $h\left(\theta_{n}\right)-\nabla l\left(\theta_{n}\right) . \nabla l\left(\theta_{n}\right)+\xi_{n}$ can be interpreted as an estimator of $\nabla l\left(\theta_{n}\right)$, while $\zeta_{n}$ and $\eta_{n}$ can be considered as the variance and bias of this estimator. Using results of martingale limit theory and the results of Steps 5 and 6 , it is shown that $\left\{\zeta_{n}\right\}_{n \geq 0}$ satisfy the Kushner-Clark noise condition. Relying on the results of Step 7, it is also shown that the asymptotic magnitude of $\left\{\eta_{n}\right\}_{n \geq 0}$ is inversely proportional to $N$ with a deterministic multiplicative constant. The details are provided in Lemma 6.1.

Step 9: Using the results presented in Section 4 and the results of [31], [32], Lemma 6.2 is proved. Then, relying on the results obtained at Step 8 and the results of [30], Theorem 2.1 is established.

\section{Example}

To illustrate the main results and their applicability, we use them to study recursive maximum likelihood estimation for the following non-linear state-space model:

$$
X_{n+1}^{\theta, \lambda}=A_{\theta}\left(X_{n}^{\theta, \lambda}\right)+B_{\theta}\left(X_{n}^{\theta, \lambda}\right) V_{n}, \quad Y_{n}^{\theta, \lambda}=C_{\theta}\left(X_{n}^{\theta, \lambda}\right)+D_{\theta}\left(X_{n}^{\theta, \lambda}\right) W_{n}, \quad n \geq 0 .
$$

Here, $\theta \in \Theta$ and $\lambda \in \mathcal{P}(\mathcal{X})$ are the parameters indexing the state-space model (20). $A_{\theta}(x)$ and $B_{\theta}(x)$ are functions which map $\theta \in \Theta, x \in \mathbb{R}^{d_{x}}$ (respectively) to $\mathbb{R}^{d_{x}}$ and $\mathbb{R}^{d_{x} \times d_{x}}$ while $C_{\theta}(x)$ and $D_{\theta}(x)$ map $\theta \in \Theta, x \in \mathbb{R}^{d_{x}}$ (respectively) to $\mathbb{R}^{d_{y}}$ and $\mathbb{R}^{d_{y} \times d_{y}} . X_{0}^{\theta, \lambda}$ is an $\mathbb{R}^{d_{x}}$-valued random variable defined on a probability space $(\Omega, \mathcal{F}, P)$ and distributed according to $\lambda .\left\{V_{n}\right\}_{n \geq 0}$ are $\mathbb{R}^{d_{x}}$-valued i.i.d. random variables distributed according the probability density $v(x)$ with respect to the Lebesgue measure while $\left\{W_{n}\right\}_{n \geq 0}$

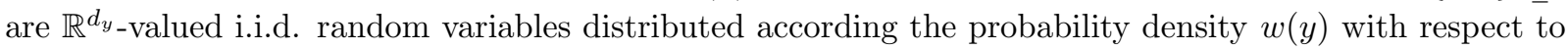
the Lebesgue measure. We also assume that $X_{0}^{\lambda},\left\{V_{n}\right\}_{n \geq 0}$ and $\left\{W_{n}\right\}_{n \geq 0}$ are (jointly) independent.

Let $p_{\theta}\left(x^{\prime} \mid x\right)$ and $q_{\theta}(y \mid x)$ be the functions defined by

$$
p_{\theta}\left(x^{\prime} \mid x\right)=\frac{v\left(B_{\theta}^{-1}(x)\left(x^{\prime}-A_{\theta}(x)\right)\right) 1_{\mathcal{X}}\left(x^{\prime}\right)}{\int_{\mathcal{X}} v\left(B_{\theta}^{-1}(x)\left(x^{\prime \prime}-A_{\theta}(x)\right)\right) d x^{\prime \prime}}, \quad q_{\theta}(y \mid x)=\frac{w\left(D_{\theta}^{-1}(x)\left(y-C_{\theta}(x)\right)\right) 1_{\mathcal{Y}}(y)}{\int_{\mathcal{Y}} w\left(D_{\theta}^{-1}(x)\left(y^{\prime}-C_{\theta}(x)\right)\right) d y^{\prime}}
$$

for $\theta \in \Theta, x, x^{\prime} \in \mathbb{R}^{d_{x}}, y \in \mathbb{R}^{d_{y}}$, where $\mathcal{X} \in \mathcal{B}\left(\mathbb{R}^{d_{x}}\right), \mathcal{Y} \in \mathcal{B}\left(\mathbb{R}^{d_{y}}\right)$. If $\mathcal{X}=\mathbb{R}^{d_{x}}, \mathcal{Y}=\mathbb{R}^{d_{y}}$, then $p_{\theta}\left(x^{\prime} \mid x\right)$ and $q_{\theta}(y \mid x)$ reduce to the conditional densities of $X_{n+1}^{\theta, \lambda}$ and $Y_{n}^{\theta, \lambda}$ (respectively) given $X_{n}^{\theta, \lambda}=x$. When $\mathcal{X} \neq \mathbb{R}^{d_{x}}, \mathcal{Y} \neq \mathbb{R}^{d_{y}}, p_{\theta}\left(x^{\prime} \mid x\right)$ and $q_{\theta}(y \mid x)$ can be viewed as a truncation of state-space model (20) to domains $\mathcal{X}$ and $\mathcal{Y}$. Due to the finite precision of digital computers, this kind of truncation is involved (explicitly or implicitly) in the implementation of any numerical approximation to the optimal filter for state-space model (20). In [15], a truncation scheme similar to (21) has been theoretically analyzed and the choice of the corresponding truncation domain has been addressed. In the context of algorithm (1) (4), the choice of domains $\mathcal{X}$ and $\mathcal{Y}$ is much more complex as it involves many factors such as the stability, accuracy, convergence and convergence rate of algorithm (1) - (4), as well as the stability and accuracy of the optimal filter for model (21). As such, the choice of $\mathcal{X}$ and $\mathcal{Y}$ is beyond the scope of this paper.

In this section, we rely on the following assumptions.

Assumption 3.1. $\mathcal{X}$ and $\mathcal{Y}$ are compact sets with non-empty interiors.

Assumption 3.2. $v(x)>0$ and $w(y)>0$ for each $x \in \mathbb{R}^{d_{x}}, y \in \mathbb{R}^{d_{y}} . B_{\theta}(x)$ and $D_{\theta}(x)$ are invertible for each $\theta \in \Theta, x \in \mathbb{R}^{d_{x}}$. 
Assumption 3.3. $v(x)$ and $w(y)$ are differentiable for each $x \in \mathbb{R}^{d_{x}}, y \in \mathbb{R}^{d_{y}}$. The first order derivatives of $v(x)$ and $w(y)$ are locally Lipschitz continuous on $\mathbb{R}^{d_{x}}, \mathbb{R}^{d_{y}} . A_{\theta}(x), B_{\theta}(x), C_{\theta}(x)$ and $D_{\theta}(x)$ are differentiable in $\theta$ for each $\theta \in \Theta, x \in \mathbb{R}^{d_{x}}$. The first order derivatives in $\theta$ of $A_{\theta}(x), B_{\theta}(x), C_{\theta}(x)$ and $D_{\theta}(x)$ are locally Lipschitz continuous in $(\theta, x)$ on $\Theta \times \mathbb{R}^{d_{x}}$.

Assumption 3.4. $v(x)$ and $w(y)$ are $p$-times differentiable for each $x \in \mathbb{R}^{d_{x}}, y \in \mathbb{R}^{d_{y}}$, where $p>d$. The $p$-th order derivatives of $v(x)$ and $w(y)$ are locally bounded on $\mathbb{R}^{d_{x}}, \mathbb{R}^{d_{y}} . A_{\theta}(x), B_{\theta}(x), C_{\theta}(x)$ and $D_{\theta}(x)$ are $p$-times differentiable in $\theta$ for each $\theta \in \Theta, x \in \mathbb{R}^{d_{x}}$. The $p$-th order derivatives in $\theta$ of $A_{\theta}(x), B_{\theta}(x)$, $C_{\theta}(x)$ and $D_{\theta}(x)$ are locally bounded in $(\theta, x)$ on $\Theta \times \mathbb{R}^{d_{x}}$.

Assumption 3.5. $v(x)$ and $w(y)$ are real-analytic for each $x \in \mathbb{R}^{d_{x}}, y \in \mathbb{R}^{d_{y}} . A_{\theta}(x), B_{\theta}(x), C_{\theta}(x)$ and $D_{\theta}(x)$ are real-analytic in $(\theta, x)$ for each $\theta \in \Theta, x \in \mathbb{R}^{d_{x}}$.

Regarding Assumptions $2.3-2.6$ and $3.1-3.5$, the following relationships can be established. Assumptions $3.1-3.3$ imply Assumptions 2.3 and 2.4, while Assumptions 3.4 and 3.5 are particular cases of Assumptions 2.5 and 2.6 (respectively). For the proof of these relationships, see [31, Corollary 4.1], [32, Corollary 4.1] (and the arguments used therein). Assumptions 3.1 - 3.5 are relevant for several practically important classes of state-space models and cover, for example, stochastic volatility models, dynamic probit models and their truncated versions. For other models satisfying (20) and Assumptions $3.1-3.5$, see [8], [9], [14] and references cited therein.

As a direct consequence of the relationships between Assumptions $2.3-2.6$ and $3.1-3.5$, we get the following corollary to Theorem 2.1.

Corollary 3.1. (i) If Assumptions 2.1, 2.2 and 3.1 - 3.3 are satisfied, then the conclusions of Part (i) of Theorem 2.1 hold.

(ii) If Assumptions 2.1, 2.2 and 3.1 - 3.4 are fulfilled, then the conclusions of Part (ii) of Theorem 2.1 hold.

(iii) If Assumptions 2.1, 2.2, 3.1 - 3.3 and 3.5 are satisfied, then the conclusions of Part (iii) of Theorem 2.1 hold.

\section{Results Related to Optimal Filter and Log-Likelihood Rate}

In this section, we study the stability and analytical properties of the optimal filter and its derivative as well as some regularity properties of the log-likelihood rate. The results presented here are a prerequisite for Lemmas 5.6 and 6.2. Note that we only consider here the results which are essential for the proof of Theorem 2.1 and not well-covered in the existing literature on optimal filtering.

Throughout this section and the whole paper, we use the following notation. $Q$ stands for any compact set satisfying $Q \subset \Theta . \mathcal{M}_{s}(\mathcal{X})$ is the collection of signed measures on $\mathcal{X}$, while $\mathcal{M}_{s}^{d}(\mathcal{X})$ is the set of $d$-dimensional vector measures on $\mathcal{X}$. For $\xi \in \mathcal{M}_{s}(\mathcal{X}),|\xi|(d x)$ and $\|\xi\|$ denote (respectively) the total variation and the total variation norm of $\xi$. For $\zeta \in \mathcal{M}_{s}^{d}(\mathcal{X}),|\zeta|(d x)$ and $\|\zeta\|$ denote (respectively) the total variation and the total variation norm of $\zeta$ induced by $l_{1}$ vector norm. ${ }^{4} r_{\theta}\left(x^{\prime} \mid y, x\right)$ is the function defined by

$$
r_{\theta}\left(x^{\prime} \mid y, x\right)=p_{\theta}\left(x^{\prime} \mid x\right) q_{\theta}(y \mid x)
$$

for $\theta \in \Theta, x, x^{\prime} \in \mathcal{X}, y \in \mathcal{Y}$, while $h_{\theta, y}(x \mid \xi, \zeta)$ and $H_{\theta, y}(\xi, \zeta)$ are defined for $\xi \in \mathcal{P}(\mathcal{X}), \zeta \in \mathcal{M}_{s}^{d}(\mathcal{X})$ as

$$
h_{\theta, y}(x \mid \xi, \zeta)=\frac{\int r_{\theta}\left(x \mid y, x^{\prime}\right) \zeta\left(d x^{\prime}\right)+\int \nabla_{\theta} r_{\theta}\left(x \mid y, x^{\prime}\right) \xi\left(d x^{\prime}\right)}{\int q_{\theta}\left(y \mid x^{\prime}\right) \xi\left(d x^{\prime}\right)}, \quad H_{\theta, y}(\xi, \zeta)=\int h_{\theta, y}(x \mid \xi, \zeta) \mu(d x) .
$$

$r_{\theta, \boldsymbol{y}}^{m: n}\left(x^{\prime} \mid x\right)$ is the function recursively defined by

$$
r_{\theta, \boldsymbol{y}}^{m: m+1}\left(x^{\prime} \mid x\right)=r_{\theta}\left(x^{\prime} \mid y_{m}, x\right), \quad r_{\theta, \boldsymbol{y}}^{m: n+1}\left(x^{\prime} \mid x\right)=\int r_{\theta, \boldsymbol{y}}^{n: n+1}\left(x^{\prime} \mid x^{\prime \prime}\right) r_{\theta, \boldsymbol{y}}^{m: n}\left(x^{\prime \prime} \mid x\right) \mu\left(d x^{\prime \prime}\right)
$$

\footnotetext{
${ }^{4}$ If $\zeta \in \mathcal{M}_{s}^{d}(\mathcal{X})$, then $|\zeta|(d x)=\sum_{i=1}^{d}\left|e_{i}^{T} \zeta\right|(d x)$ and $\|\zeta\|=\sum_{i=1}^{d}\left\|e_{i}^{T} \zeta\right\|$, where $e_{i}$ is the $i$-th standard unit vector in $\mathbb{R}^{d}$.
} 
for $n>m \geq 0$ and a sequence $\boldsymbol{y}=\left\{y_{n}\right\}_{n \geq 0}$ in $\mathcal{Y} . h_{\theta, \boldsymbol{y}}^{m: n}(x \mid \xi, \zeta)$ and $H_{\theta, \boldsymbol{y}}^{m: n}(\xi, \zeta)$ are the functions defined by

$$
h_{\theta, \boldsymbol{y}}^{m: n}(x \mid \xi, \zeta)=\frac{\int r_{\theta, \boldsymbol{y}}^{m: n}\left(x \mid x^{\prime}\right) \zeta\left(d x^{\prime}\right)+\int \nabla_{\theta} r_{\theta, \boldsymbol{y}}^{m: n}\left(x \mid x^{\prime}\right) \xi\left(d x^{\prime}\right)}{\iint r_{\theta, \boldsymbol{y}}^{m: n}\left(x^{\prime \prime} \mid x^{\prime}\right) \xi\left(d x^{\prime}\right) \mu\left(d x^{\prime \prime}\right)}, \quad H_{\theta, \boldsymbol{y}}^{m: n}(\xi, \zeta)=\int h_{\theta, \boldsymbol{y}}^{m: n}(x \mid \xi, \zeta) \mu(d x),
$$

while $f_{\theta, \boldsymbol{y}}^{m: n}(x \mid \xi)$ and $g_{\theta, \boldsymbol{y}}^{m: n}(x \mid \xi, \zeta)$ are defined as

$$
f_{\theta, \boldsymbol{y}}^{m: n}(x \mid \xi)=\frac{\int r_{\theta, \boldsymbol{y}}^{m: n}\left(x \mid x^{\prime}\right) \xi\left(d x^{\prime}\right)}{\iint r_{\theta, \boldsymbol{y}}^{m: n}\left(x^{\prime \prime} \mid x^{\prime}\right) \xi\left(d x^{\prime}\right) \mu\left(d x^{\prime \prime}\right)}, \quad g_{\theta, \boldsymbol{y}}^{m: n}(x \mid \xi, \zeta)=h_{\theta, \boldsymbol{y}}^{m: n}(x \mid \xi, \zeta)-f_{\theta, \boldsymbol{y}}^{m: n}(x \mid \xi) H_{\theta, \boldsymbol{y}}^{m: n}(\xi, \zeta) .
$$

$F_{\theta, \boldsymbol{y}}^{m: m}(d x \mid \xi), F_{\theta, \boldsymbol{y}}^{m: n}(d x \mid \xi)$ and $G_{\theta, \boldsymbol{y}}^{m: m}(d x \mid \xi, \zeta), G_{\theta, \boldsymbol{y}}^{m: n}(d x \mid \xi, \zeta)$ are the measures defined for $B \in \mathcal{B}(\mathcal{X})$ by

$$
\begin{aligned}
F_{\theta, \boldsymbol{y}}^{m: m}(B \mid \xi) & =\xi(B), \quad F_{\theta, \boldsymbol{y}}^{m: n}(B \mid \xi)=\int_{B} f_{\theta, \boldsymbol{y}}^{m: n}(x \mid \xi) \mu(d x), \\
G_{\theta, \boldsymbol{y}}^{m: m}(B \mid \xi, \zeta) & =\zeta(B), \quad G_{\theta, \boldsymbol{y}}^{m: n}(B \mid \xi, \zeta)=\int_{B} g_{\theta, \boldsymbol{y}}^{m: n}(x \mid \xi, \zeta) \mu(d x) .
\end{aligned}
$$

Throughout this paper, the measures $F_{\theta, \boldsymbol{y}}^{m: n}(d x \mid \xi)$ and $G_{\theta, \boldsymbol{y}}^{m: n}(d x \mid \xi, \zeta)$ are also denoted by $F_{\theta, \boldsymbol{y}}^{m: n}(\xi)$ and $G_{\theta, \boldsymbol{y}}^{m: n}(\xi, \zeta)$ (short-hand notation). Then, it is easy to show that $F_{\theta, \boldsymbol{y}}^{m: n}(\xi)$ and $G_{\theta, \boldsymbol{y}}^{m: n}(\xi, \zeta)$ are the optimal (one-step) predictor and its gradient, i.e.,

$$
F_{\theta, \boldsymbol{y}}^{0: n}(B \mid \lambda)=P\left(X_{n}^{\theta, \lambda} \in B \mid Y_{0: n-1}^{\theta, \lambda}=y_{0: n-1}\right), \quad G_{\theta, \boldsymbol{y}}^{0: n}(B \mid \lambda, \mathbf{0})=\nabla_{\theta} F_{\theta, \boldsymbol{y}}^{0: n}(B \mid \lambda)
$$

for each $\lambda \in \mathcal{P}(\mathcal{X}), n \geq 1$. Here, $\mathbf{0}$ is the $d$-dimensional zero-measure (i.e., $\mathbf{0} \in \mathcal{M}_{s}^{d}(\mathcal{X}),\|\mathbf{0}\|=0$ ).

Lemma 4.1. Let Assumptions 2.2-2.4 hold. Then, the following is true:

(i) $l(\theta)$ is well-defined and differentiable on $\Theta$.

(ii) $\nabla l(\theta)$ is locally Lipschitz continuous on $\Theta$ and satisfies

$$
\nabla l(\theta)=\lim _{n \rightarrow \infty} E\left(H_{\theta, Y_{n}}\left(F_{\theta, \boldsymbol{Y}}^{0: n}(\xi), G_{\theta, \boldsymbol{Y}}^{0: n}(\xi, \zeta)\right)\right)
$$

for all $\theta \in \Theta, \xi \in \mathcal{P}(\mathcal{X}), \zeta \in \mathcal{M}_{s}^{d}(\mathcal{X})$, where $\boldsymbol{Y}=\left\{Y_{n}\right\}_{n \geq 0}$.

(iii) There exists a real number $C_{1, Q} \in[1, \infty)$ (depending only on $p_{\theta}\left(x^{\prime} \mid x\right), q_{\theta}(y \mid x)$ ) such that

$$
\left\|G_{\theta, \boldsymbol{y}}^{0: n}(\xi, \zeta)\right\| \leq C_{1, Q}(1+\|\zeta\|)
$$

for all $\theta \in Q, \xi \in \mathcal{P}(\mathcal{X}), \zeta \in \mathcal{M}_{s}^{d}(\mathcal{X}), n \geq 0$ and any sequence $\boldsymbol{y}=\left\{y_{n}\right\}_{n \geq 0}$.

Lemma 4.1 is proved in Appendix 2.

\section{Results Related to Sequential Monte Carlo Approximations}

In this section, we study the asymptotic properties of the particles $\left\{\hat{X}_{n, i}: n \geq 0,1 \leq i \leq N\right\}$ and their weights $\left\{W_{n, i}: n \geq 0,1 \leq i \leq N\right\}$. Using these properties, we show that the Poisson equation associated with algorithm (1) - (4) has a Lipschitz continuous solution (see Lemma 5.4). The results presented here are needed to analyze the error in the Monte Carlo estimation of $\nabla l(\theta)$ (see Lemma 6.1 and (19)).

Throughout this section, we use the following notation. $\mathcal{V}$ and $\mathcal{Z}$ are the sets defined by $\mathcal{V}=\mathcal{Y} \times \mathcal{X} \times \mathcal{X}^{N}$ and $\mathcal{Z}=\mathcal{V} \times \mathbb{R}^{d \times N}$. $e$ is the $N$-dimensional vector whose all elements are one (i.e., $e=(1, \ldots, 1)^{T} \in \mathbb{R}^{N}$ ). $I$ is the $N \times N$ unit matrix, while $\Lambda$ is the $N \times N$ matrix defined as

$$
\Lambda=I-\frac{e e^{T}}{N} .
$$

$A_{\theta}\left(v, v^{\prime}\right)$ and $B_{\theta}\left(v, v^{\prime}\right)$ are respectively $\mathbb{R}^{N \times N}$ and $\mathbb{R}^{d \times N}$-valued functions defined by

$$
A_{\theta}^{i, j}\left(v, v^{\prime}\right)=\frac{r_{\theta}\left(x_{j}^{\prime} \mid y, x_{i}\right)}{\sum_{k=1}^{N} r_{\theta}\left(x_{j}^{\prime} \mid y, x_{k}\right)}, \quad B_{\theta}^{j}\left(v, v^{\prime}\right)=\frac{\sum_{k=1}^{N} \nabla_{\theta} r_{\theta}\left(x_{j}^{\prime} \mid y, x_{k}\right)}{\sum_{k=1}^{N} r_{\theta}\left(x_{j}^{\prime} \mid y, x_{k}\right)}
$$


for $\theta \in \Theta, x, x^{\prime} \in \mathcal{X}, y, y^{\prime} \in \mathcal{Y}, \hat{x}=\left(x_{1}, \ldots, x_{N}\right) \in \mathcal{X}^{N}, \hat{x}^{\prime}=\left(x_{1}^{\prime}, \ldots, x_{N}^{\prime}\right) \in \mathcal{X}^{N}, 1 \leq i, j \leq N$ and $v=(y, x, \hat{x}), v^{\prime}=\left(y^{\prime}, x^{\prime}, \hat{x}^{\prime}\right)$, where $A_{\theta}^{i, j}\left(v, v^{\prime}\right)$ and $B_{\theta}^{j}\left(v, v^{\prime}\right)$ are the $(i, j)$-entry of $A_{\theta}\left(v, v^{\prime}\right)$ and the $j$-th column of $B_{\theta}\left(v, v^{\prime}\right)$ (respectively). $C_{\theta}(v)$ and $D_{\theta}(v)$ are respectively $\mathbb{R}^{N}$ and $\mathbb{R}^{d}$-valued functions defined by

$$
C_{\theta}^{i}(v)=\frac{q_{\theta}\left(y \mid x_{i}\right)}{\sum_{k=1}^{N} q_{\theta}\left(y \mid x_{k}\right)}-\frac{1}{N}, \quad D_{\theta}(v)=\frac{\sum_{k=1}^{N} \nabla_{\theta} q_{\theta}\left(y \mid x_{k}\right)}{\sum_{k=1}^{N} q_{\theta}\left(y \mid x_{k}\right)},
$$

where $C_{\theta}^{i}(v)$ is the $i$-th element of $C_{\theta}(v) . H(\theta, z)$ is the function defined by

$$
H(\theta, z)=W C_{\theta}(v)+D_{\theta}(v)
$$

for $v \in \mathcal{V}, W \in \mathbb{R}^{d \times N}$ and $z=(v, W)$. Then, it is straightforward to verify

$$
e^{T} A_{\theta}\left(v, v^{\prime}\right)=e^{T}, \quad e^{T} C_{\theta}(v)=0
$$

for all $\theta \in \Theta, v, v^{\prime} \in \mathcal{V}$, where $A_{\theta}\left(v, v^{\prime}\right), C_{\theta}(v)$ are defined in (27), (28).

We rely here on the following notation, too. $s_{\theta}(x \mid y, \hat{x})$ is the function defined by

$$
s_{\theta}(x \mid y, \hat{x})=\frac{\sum_{k=1}^{N} p_{\theta}\left(x \mid x_{k}\right) q_{\theta}\left(y \mid x_{k}\right)}{\sum_{k=1}^{N} q_{\theta}\left(y \mid x_{k}\right)} .
$$

For $\hat{x}=\left(x_{1}, \ldots, x_{N}\right) \in \mathcal{X}^{N}, S_{\theta}\left(d \hat{x}^{\prime} \mid y, \hat{x}\right)$ is the conditional probability measure on $\mathcal{X}^{N}$ defined for $B \in$ $\mathcal{B}\left(\mathcal{X}^{N}\right)$ as

$$
S_{\theta}(B \mid y, \hat{x})=\int \cdots \int I_{B}\left(x_{1}^{\prime}, \ldots, x_{N}^{\prime}\right)\left(\prod_{k=1}^{N} s_{\theta}\left(x_{k}^{\prime} \mid y, \hat{x}\right)\right) \mu\left(d x_{1}^{\prime}\right) \cdots \mu\left(d x_{N}^{\prime}\right),
$$

where $I_{B}$ denotes the indicator of $B . T_{\theta}\left(v, d v^{\prime}\right)$ is the kernel on $\mathcal{V}$ defined for $B \in \mathcal{B}(\mathcal{V})$ and $v=(y, x, \hat{x})$ by

$$
T_{\theta}(v, B)=\iiint I_{B}\left(y^{\prime}, x^{\prime}, \hat{x}^{\prime}\right) Q\left(x^{\prime}, d y^{\prime}\right) P\left(x, d x^{\prime}\right) S_{\theta}\left(d \hat{x}^{\prime} \mid y, \hat{x}\right) .
$$

$\Pi_{\theta}\left(z, d z^{\prime}\right)$ is the kernel on $\mathcal{Z}$ defined for $B \in \mathcal{B}(\mathcal{Z}), W \in \mathbb{R}^{d \times N}$ and $z=(v, W)$ as

$$
\Pi_{\theta}(z, B)=\int I_{B}\left(v^{\prime}, W A_{\theta}\left(v, v^{\prime}\right)+B_{\theta}\left(v, v^{\prime}\right)\right) T_{\theta}\left(v, d v^{\prime}\right) .
$$

Then, it is straightforward to verify that $\left\{V_{n}\right\}_{n \geq 0}$ and $\left\{Z_{n}\right\}_{n \geq 0}$ defined in (16) satisfy

$$
\begin{aligned}
& P\left(V_{n+1} \in A \mid \theta_{0}, V_{0}, \ldots, \theta_{n}, V_{n}\right)=T_{\theta_{n}}\left(V_{n}, A\right), \\
& P\left(Z_{n+1} \in B \mid \theta_{0}, Z_{0}, \ldots, \theta_{n}, Z_{n}\right)=\Pi_{\theta_{n}}\left(Z_{n}, B\right)
\end{aligned}
$$

almost surely for each $A \in \mathcal{B}(\mathcal{V}), B \in \mathcal{B}(\mathcal{Z}), n \geq 0$.

Using functions $A_{\theta}\left(v, v^{\prime}\right), B_{\theta}\left(v, v^{\prime}\right)$ and $S_{\theta}\left(d \hat{x}^{\prime} \mid y, \hat{x}\right)$, we introduce the following notation. $\left\{\hat{X}_{n, i}^{\theta}: n \geq\right.$ $0,1 \leq i \leq N\}$ are $\mathcal{X}$-valued random variables generated through the sequential Monte Carlo scheme

$$
\hat{X}_{n+1, i}^{\theta} \sim s_{\theta}\left(x \mid Y_{n},\left(\hat{X}_{n, 1}^{\theta}, \ldots, \hat{X}_{n, N}^{\theta}\right)\right) \mu(d x),
$$

while $\hat{X}_{n}^{\theta}, V_{n}^{\theta}$ are the random variables defined by

$$
\hat{X}_{n}^{\theta}=\left(\hat{X}_{n, 1}^{\theta}, \ldots, \hat{X}_{n, N}^{\theta}\right), \quad V_{n}^{\theta}=\left(Y_{n}, X_{n}, \hat{X}_{n}^{\theta}\right) .
$$

In (36), $\left\{\hat{X}_{n+1, i}^{\theta}: 1 \leq i \leq N\right\}$ are sampled independently from one another and independently of $\left\{X_{k}\right.$ : $0 \leq k \leq n\},\left\{Y_{k}, \hat{X}_{k, i}^{\theta}: 0 \leq k<n, 1 \leq i \leq N\right\}$, while $\left\{\hat{X}_{0, i}^{\theta}: 1 \leq i \leq N\right\}$ are selected independently of $\left(X_{0}, Y_{0}\right) .\left\{W_{n}^{\theta}\right\}_{n \geq 0}$ are $d \times N$ random matrices generated by the recursion

$$
W_{n+1}^{\theta}=W_{n}^{\theta} A_{\theta}\left(V_{n}^{\theta}, V_{n+1}^{\theta}\right)+B_{\theta}\left(V_{n}^{\theta}, V_{n+1}^{\theta}\right),
$$


while $Z_{n}^{\theta}$ is the random variable defined by $Z_{n}^{\theta}=\left(V_{n}^{\theta}, W_{n}^{\theta}\right)$. In (37), $W_{0}^{\theta}$ is selected independently of $\left(X_{0}, Y_{0}\right)$. Then, it can easily be shown that $\left\{V_{n}^{\theta}\right\}_{n \geq 0}$ and $\left\{Z_{n}^{\theta}\right\}_{n \geq 0}$ are Markov chains whose transition kernels are $T_{\theta}\left(v, d v^{\prime}\right)$ and $\Pi_{\theta}\left(z, d z^{\prime}\right)$ (respectively).

Using functions $A_{\theta}\left(v, v^{\prime}\right), B_{\theta}\left(v, v^{\prime}\right), C_{\theta}(v), D_{\theta}(v)$ (defined in (27), (28)) and stochastic process $\left\{V_{n}^{\theta}\right\}_{n \geq 0}$, we introduce the following notation. $T_{\theta}^{n}\left(v, d v^{\prime}\right)$ and $\tau_{\theta}(d v)$ are (respectively) the $n$-th step transition kernel and the invariant probability measure of $\left\{V_{n}^{\theta}\right\}_{n \geq 0}$ (the existence and uniqueness of $\tau_{\theta}(d v)$ are guaranteed by Lemma 5.1). $\tilde{T}_{\theta}^{n}\left(v, d v^{\prime}\right)$ is the kernel on $\mathcal{V}$ defined for $B \in \mathcal{B}(\mathcal{V})$ by

$$
\tilde{T}_{\theta}^{n}(v, B)=T_{\theta}^{n}(v, B)-\tau_{\theta}(B) .
$$

$\tilde{A}_{\theta}^{0}(v)$ and $\Phi_{\theta}^{0}(v)$ are the functions defined by

$$
\tilde{A}_{\theta}^{0}(v)=I, \quad \Phi_{\theta}^{0}(v)=C_{\theta}(v) .
$$

$\tilde{A}_{\theta}^{n}\left(v_{0}, \ldots, v_{n}\right)$ and $\Phi_{\theta}^{n}(v)$ are the functions defined for $v, v_{0}, \ldots, v_{n} \in \mathcal{V}, n \geq 1$ by

$$
\tilde{A}_{\theta}^{n}\left(v_{0}, \ldots, v_{n}\right)=A_{\theta}\left(v_{0}, v_{1}\right) \cdots A_{\theta}\left(v_{n-1}, v_{n}\right), \quad \Phi_{\theta}^{n}(v)=E\left(\tilde{A}_{\theta}^{n}\left(V_{0}^{\theta}, \ldots, V_{n}^{\theta}\right) C_{\theta}\left(V_{n}^{\theta}\right) \mid V_{0}^{\theta}=v\right) .
$$

$\tilde{B}_{\theta}^{n}\left(v_{0}, \ldots, v_{n}\right)$ and $\Psi_{\theta}^{n}(v)$ are the functions defined by

$$
\tilde{B}_{\theta}^{n}\left(v_{0}, \ldots, v_{n}\right)=B_{\theta}\left(v_{0}, v_{1}\right) \tilde{A}_{\theta}^{n-1}\left(v_{1}, \ldots, v_{n}\right), \quad \Psi_{\theta}^{n}(v)=E\left(\tilde{B}_{\theta}^{n}\left(V_{0}^{\theta}, \ldots, V_{n}^{\theta}\right) C_{\theta}\left(V_{n}^{\theta}\right) \mid V_{0}^{\theta}=v\right) .
$$

$h(\theta)$ is the function defined by

$$
h(\theta)=\int D_{\theta}(v) \tau_{\theta}(d v)+\sum_{n=1}^{\infty} \int \Psi_{\theta}^{n}(v) \tau_{\theta}(d v) .
$$

Then, for each $\theta \in \Theta, v \in \mathcal{V}, n \geq 1$, it is straightforward to verify

$$
\Psi_{\theta}^{n}(v)=E\left(B_{\theta}\left(V_{0}^{\theta}, V_{1}^{\theta}\right) \Phi_{\theta}^{n}\left(V_{1}^{\theta}\right) \mid V_{0}^{\theta}=v\right) .
$$

Remark. Throughout this and subsequent sections, the following convention is applied. Diacritic $\sim$ is used to denote a locally defined quantity, i.e., a quantity whose definition holds only within the proof where the quantity appears. We also recall here that $Q$ stands for any compact set satisfying $Q \subset \Theta$.

Lemma 5.1. Let Assumptions 2.2 - 2.4 hold. Then, the following is true:

(i) $\left\{V_{n}^{\theta}\right\}_{n \geq 0}$ is geometrically ergodic for each $\theta \in \Theta$.

(ii) There exist real numbers $\rho_{1, Q} \in(0,1), C_{2, Q} \in[1, \infty)$ (possibly depending on $N$ ) such that

$$
\begin{aligned}
& \left|\tilde{T}_{\theta}^{n}(v, B)\right| \leq C_{2, Q} \rho_{1, Q}^{n}, \\
& \left|\tilde{T}_{\theta}^{n}(v, B)-\tilde{T}_{\theta^{\prime}}^{n}(v, B)\right| \leq C_{2, Q} \rho_{1, Q}^{n}\left\|\theta-\theta^{\prime}\right\|, \\
& \quad \max \left\{\left|\tau_{\theta}(B)-\tau_{\theta^{\prime}}(B)\right|,\left|T_{\theta}(v, B)-T_{\theta^{\prime}}(v, B)\right|\right\} \leq C_{2, Q}\left\|\theta-\theta^{\prime}\right\|
\end{aligned}
$$

for all $\theta, \theta^{\prime} \in Q, v \in \mathcal{V}, B \in \mathcal{B}(\mathcal{V}), n \geq 0$.

Proof. Using Assumption 2.2 and [22, Theorem 16.0.2], we conclude that there exist an integer $n_{0} \geq 1$, a real number $\gamma \in(0,1)$ and a probability measure $\xi(d x)$ on $\mathcal{X}$ such that $P^{n_{0}}(x, A) \geq \gamma \xi(A)$ for all $x \in \mathcal{X}$, $A \subseteq \mathcal{B}(\mathcal{X})$.

Throughout the proof, the following notation is used. $\theta, \theta^{\prime}$ are any elements of $Q . x, x_{1}, \ldots, x_{N}$ are any elements of $\mathcal{X}$, while $\hat{x}=\left(x_{1}, \ldots, x_{N}\right) . y$ is any element of $\mathcal{Y}$, while $v=(y, x, \hat{x}) . B$ is any element of $\mathcal{B}(\mathcal{V})$, while $n$ is any non-negative integer. $\zeta(d \hat{x})$ is the probability measure on $\mathcal{X}^{N}$ defined for $A \in \mathcal{B}\left(\mathcal{X}^{N}\right)$ by

$$
\zeta(A)=\left(\frac{1}{\mu(\mathcal{X})}\right)^{N} \int \cdots \int I_{A}\left(x_{1}, \ldots, x_{N}\right) \mu\left(d x_{1}\right) \cdots \mu\left(d x_{N}\right)
$$


Let $\beta_{Q}=\left(\varepsilon_{Q} \mu(\mathcal{X})\right)^{N}$ ( $\varepsilon_{Q}$ is specified in Assumption 2.3, while $\mu(d x)$ is defined in Subsection 2.1). Relying on Assumption 2.3, we deduce

$$
\varepsilon_{Q} \leq s_{\theta}(x \mid y, \hat{x}) \leq \frac{1}{\varepsilon_{Q}} .
$$

Consequently, for $A \in \mathcal{B}\left(\mathcal{X}^{N}\right)$, we get

$$
S_{\theta}(A \mid y, \hat{x}) \geq \varepsilon_{Q}^{N} \int \cdots \int I_{A}\left(x_{1}^{\prime}, \ldots, x_{N}^{\prime}\right) \mu\left(d x_{1}^{\prime}\right) \cdots \mu\left(d x_{N}^{\prime}\right)=\beta_{Q} \zeta(A) .
$$

Hence, we have

$$
\begin{aligned}
T_{\theta}(v, B) & =\iiint I_{B}\left(y^{\prime}, x^{\prime}, \hat{x}^{\prime}\right) Q\left(x^{\prime}, d y^{\prime}\right) P\left(x, d x^{\prime}\right) S_{\theta}\left(d \hat{x}^{\prime} \mid y, \hat{x}\right) \\
& \geq \beta_{Q} \iiint I_{B}\left(y^{\prime}, x^{\prime}, \hat{x}^{\prime}\right) Q\left(x, d y^{\prime}\right) P\left(x, d x^{\prime}\right) \zeta\left(d \hat{x}^{\prime}\right) .
\end{aligned}
$$

Therefore, we get

$$
\begin{aligned}
T_{\theta}^{n+1}(v, B) & =E\left(T_{\theta}\left(V_{n}^{\theta}, B\right) \mid V_{0}^{\theta}=v\right) \\
& \geq \beta_{Q}\left(\iiint I_{B}\left(y^{\prime}, x^{\prime}, \hat{x}^{\prime}\right) Q\left(x^{\prime}, d y^{\prime}\right) P\left(X_{n}, d x^{\prime}\right) \zeta\left(d \hat{x}^{\prime}\right) \mid Y_{0}=y, X_{0}=x, \hat{X}_{0}^{\theta}=\hat{x}\right) \\
& =\beta_{Q} \iiint I_{B}\left(y^{\prime}, x^{\prime}, \hat{x}^{\prime}\right) Q\left(x^{\prime}, d y^{\prime}\right) P^{n+1}\left(x, d x^{\prime}\right) \zeta\left(d \hat{x}^{\prime}\right) .
\end{aligned}
$$

Since $P^{n_{0}}(x, A) \geq \gamma \xi(A)$ for any $A \subseteq \mathcal{B}(\mathcal{X})$, we get

$$
T_{\theta}^{n_{0}}(v, B) \geq \beta_{Q} \gamma \iiint I_{B}\left(y^{\prime}, x^{\prime}, \hat{x}^{\prime}\right) Q\left(x^{\prime}, d y^{\prime}\right) \xi\left(d x^{\prime}\right) \zeta\left(d \hat{x}^{\prime}\right) .
$$

Let $\rho_{1, Q}=\left(1-\beta_{Q} \gamma\right)^{1 /\left(2 n_{0}\right)}$. As $v$ is any element in $\mathcal{V},[22$, Theorem 16.0.2]) and (48) imply that $\left\{V_{n}^{\theta}\right\}_{n \geq 0}$ is geometrically ergodic. The same arguments also imply

$$
\left|\tilde{T}_{\theta}^{n}(v, B)\right|=\left|T_{\theta}^{n}(v, B)-\tau_{\theta}(B)\right| \leq \rho_{1, Q}^{2 n} .
$$

Since $Q$ is any compact set in $\Theta$, we conclude that (i) is true.

Let $\tilde{C}_{1, Q}=3 \varepsilon_{Q}^{-2} K_{1, Q}, \tilde{C}_{2, Q}=\varepsilon_{Q}^{-N} \tilde{C}_{1, Q} N, \tilde{C}_{3, Q}=(\mu(\mathcal{X}))^{N} \tilde{C}_{2, Q}$ (K $K_{1, Q}$ is specified in Assumption 2.4). Owing to Assumptions 2.3, 2.4, we have

$$
\begin{aligned}
\left|s_{\theta}(x \mid y, \hat{x})-s_{\theta^{\prime}}(x \mid y, \hat{x})\right| \leq & \frac{\sum_{i=1}^{N}\left|p_{\theta}\left(x \mid x_{i}\right)-p_{\theta^{\prime}}\left(x \mid x_{i}\right)\right| q_{\theta}\left(y \mid x_{i}\right)}{\sum_{i=1}^{N} q_{\theta}\left(y \mid x_{i}\right)}+\frac{\sum_{i=1}^{N} p_{\theta^{\prime}}\left(x \mid x_{i}\right)\left|q_{\theta}\left(y \mid x_{i}\right)-q_{\theta^{\prime}}\left(y \mid x_{i}\right)\right|}{\sum_{i=1}^{N} q_{\theta}\left(y \mid x_{i}\right)} \\
& +\frac{s_{\theta^{\prime}}(x \mid y, \hat{x}) \sum_{i=1}^{N}\left|q_{\theta}\left(y \mid x_{i}\right)-q_{\theta^{\prime}}\left(y \mid x_{i}\right)\right|}{\sum_{i=1}^{N} q_{\theta}\left(y \mid x_{i}\right)} \\
\leq & \frac{3 K_{1, Q}\left\|\theta-\theta^{\prime}\right\|}{\varepsilon_{Q}^{2}}=\tilde{C}_{1, Q}\left\|\theta-\theta^{\prime}\right\| .
\end{aligned}
$$

Consequently, for any $x_{1}^{\prime}, \ldots, x_{N}^{\prime} \in \mathcal{X},(47)$ yields

$$
\begin{aligned}
&\left|\prod_{i=1}^{N} s_{\theta}\left(x_{i}^{\prime} \mid y, \hat{x}\right)-\prod_{i=1}^{N} s_{\theta^{\prime}}\left(x_{i}^{\prime} \mid y, \hat{x}\right)\right| \leq \sum_{i=1}^{N}\left(\prod_{j=1}^{i-1} s_{\theta}\left(x_{j}^{\prime} \mid y, \hat{x}\right)\right)\left(\prod_{j=i+1}^{N} s_{\theta^{\prime}}\left(x_{j}^{\prime} \mid y, \hat{x}\right)\right) \\
& \cdot\left|s_{\theta}\left(x_{i}^{\prime} \mid y, \hat{x}\right)-s_{\theta^{\prime}}\left(x_{i}^{\prime} \mid y, \hat{x}\right)\right| \\
& \leq \frac{\tilde{C}_{1, Q} N\left\|\theta-\theta^{\prime}\right\|}{\varepsilon_{Q}^{N-1}} \leq \tilde{C}_{2, Q}\left\|\theta-\theta^{\prime}\right\| .
\end{aligned}
$$


Here and throughout the paper, we use the convention that the product $\prod_{i=k}^{l}$ is one whenever $k>l$. Hence, we have

$$
\begin{aligned}
&\left|S_{\theta}(B \mid y, \hat{x})-S_{\theta^{\prime}}(B \mid y, \hat{x})\right| \leq \int \cdots \int I_{B}\left(x_{1}, \ldots, x_{N}\right)\left|\prod_{i=1}^{N} s_{\theta}\left(x_{i} \mid y, \hat{x}\right)-\prod_{i=1}^{N} s_{\theta^{\prime}}\left(x_{i} \mid y, \hat{x}\right)\right| \\
& \cdot \mu\left(d x_{1}\right) \cdots \mu\left(d x_{N}\right) \\
& \leq \tilde{C}_{2, Q}(\mu(\mathcal{X}))^{N}\left\|\theta-\theta^{\prime}\right\|=\tilde{C}_{3, Q}\left\|\theta-\theta^{\prime}\right\| .
\end{aligned}
$$

Therefore, we get

$$
\left|T_{\theta}(v, B)-T_{\theta^{\prime}}(v, B)\right| \leq \iiint I_{B}\left(y^{\prime}, x^{\prime}, \hat{x}^{\prime}\right) Q\left(x^{\prime}, d y^{\prime}\right) P\left(x, d x^{\prime}\right)\left|S_{\theta}-S_{\theta^{\prime}}\right|\left(d \hat{x}^{\prime} \mid y, \hat{x}\right) \leq \tilde{C}_{3, Q}\left\|\theta-\theta^{\prime}\right\| .
$$

Here, $\left|S_{\theta}-S_{\theta^{\prime}}\right|\left(d \hat{x}^{\prime} \mid y, \hat{x}\right)$ denotes the total variation of the signed measure $S_{\theta}\left(d \hat{x}^{\prime} \mid y, \hat{x}\right)-S_{\theta^{\prime}}\left(d \hat{x}^{\prime} \mid y, \hat{x}\right)$.

Let $\tilde{C}_{4, Q} \in[1, \infty)$ be an upper bound of sequence $\left\{n \rho_{1, Q}^{n-1}\right\}_{n \geq 1}$, while $C_{2, Q}=2 \tilde{C}_{3, Q} \tilde{C}_{4, Q}\left(1-\rho_{1, Q}\right)^{-1}$. Using (49), (50), we conclude

$$
\begin{aligned}
\left|T_{\theta}^{n+1}(v, B)-T_{\theta^{\prime}}^{n+1}(v, B)\right| & =\left|\sum_{i=0}^{n} \iint \tilde{T}_{\theta}^{i}\left(v^{\prime \prime}, B\right)\left(T_{\theta}-T_{\theta^{\prime}}\right)\left(v^{\prime}, d v^{\prime \prime}\right) T_{\theta^{\prime}}^{n-i}\left(v, d v^{\prime}\right)\right| \\
& \leq \sum_{i=0}^{n} \iint\left|\tilde{T}_{\theta}^{i}\left(v^{\prime \prime}, B\right) \| T_{\theta}-T_{\theta^{\prime}}\right|\left(v^{\prime}, d v^{\prime \prime}\right) T_{\theta^{\prime}}^{n-i}\left(v, d v^{\prime}\right) \\
& \leq \tilde{C}_{3, Q}\left\|\theta-\theta^{\prime}\right\| \sum_{i=0}^{n} \rho_{1, Q}^{2 i} \leq C_{2, Q}\left\|\theta-\theta^{\prime}\right\| .
\end{aligned}
$$

Similarly, we deduce

$$
\begin{aligned}
\left|\tilde{T}_{\theta}^{n+1}(v, B)-\tilde{T}_{\theta^{\prime}}^{n+1}(v, B)\right| & =\left|\sum_{i=0}^{n} \iint \tilde{T}_{\theta}^{i}\left(v^{\prime \prime}, B\right)\left(T_{\theta}-T_{\theta^{\prime}}\right)\left(v^{\prime}, d v^{\prime \prime}\right) \tilde{T}_{\theta^{\prime}}^{n-i}\left(v, d v^{\prime}\right)\right| \\
& \leq \sum_{i=0}^{n} \iint\left|\tilde{T}_{\theta}^{i}\left(v^{\prime \prime}, B\right) \| T_{\theta}-T_{\theta^{\prime}}\right|\left(v^{\prime}, d v^{\prime \prime}\right)\left|\tilde{T}_{\theta^{\prime}}^{n-i}\right|\left(v, d v^{\prime}\right) \\
& \leq \tilde{C}_{3, Q} \rho_{1, Q}^{2 n}(n+1)\left\|\theta-\theta^{\prime}\right\| \leq C_{2, Q} \rho_{1, Q}^{n+1}\left\|\theta-\theta^{\prime}\right\| .
\end{aligned}
$$

Combining (49), (51), we get

$$
\left|\tau_{\theta}(B)-\tau_{\theta^{\prime}}(B)\right| \leq\left|T_{\theta}^{n}(v, B)-T_{\theta^{\prime}}^{n}(v, B)\right|+\left|\tilde{T}_{\theta}^{n}(v, B)\right|+\left|\tilde{T}_{\theta^{\prime}}^{n}(v, B)\right| \leq C_{2, Q}\left\|\theta-\theta^{\prime}\right\|+2 \rho_{1, Q}^{n} .
$$

Letting $n \rightarrow \infty$ in (53) and using (49), (50), (52), we conclude that (44) - (46) hold.

Lemma 5.2. Let Assumptions 2.3 and 2.4 hold. Then, the following is true:

(i) There exists a real number $\rho_{2, Q} \in(0,1)$ (independent of $N$ and depending only on $p_{\theta}\left(x^{\prime} \mid x\right), q_{\theta}(y \mid x)$ ) such that $A_{\theta}^{i, j}\left(v, v^{\prime}\right) \geq \rho_{2, Q} / N$ for all $\theta \in Q, v, v^{\prime} \in \mathcal{V}, 1 \leq i, j \leq N$.

(ii) There exists a real number $C_{3, Q} \in[1, \infty$ ) (possibly depending on $N$ ) such that

$$
\begin{aligned}
& \max \left\{\left\|A_{\theta}\left(v, v^{\prime}\right)\right\|,\left\|B_{\theta}\left(v, v^{\prime}\right)\right\|,\left\|C_{\theta}(v)\right\|,\left\|D_{\theta}(v)\right\|\right\} \leq C_{3, Q}, \\
& \max \left\{\left\|A_{\theta}\left(v, v^{\prime}\right)-A_{\theta^{\prime}}\left(v, v^{\prime}\right)\right\|,\left\|B_{\theta}\left(v, v^{\prime}\right)-B_{\theta^{\prime}}\left(v, v^{\prime}\right)\right\|\right\} \leq C_{3, Q}\left\|\theta-\theta^{\prime}\right\|, \\
& \max \left\{\left\|C_{\theta}(v)-C_{\theta^{\prime}}(v)\right\|,\left\|D_{\theta}(v)-D_{\theta^{\prime}}(v)\right\|\right\} \leq C_{3, Q}\left\|\theta-\theta^{\prime}\right\|
\end{aligned}
$$

for all $\theta, \theta^{\prime} \in Q, v, v^{\prime} \in \mathcal{V}$.

Proof. Throughout the proof, the following notation is used. $\theta, \theta^{\prime}$ are any elements of $Q$. $x, x^{\prime}$, $x_{1}, x_{1}^{\prime}, \ldots, x_{N}, x_{N}^{\prime}$ are any elements of $\mathcal{X}$, while $\hat{x}=\left(x_{1}, \ldots, x_{N}\right), \hat{x}^{\prime}=\left(x_{1}^{\prime}, \ldots, x_{N}^{\prime}\right) . y, y^{\prime}$ are any elements of $\mathcal{Y}$, while $v=(y, x, \hat{x}), v^{\prime}=\left(y^{\prime}, x^{\prime}, \hat{x}^{\prime}\right) . i, j$ are any integers satisfying $1 \leq i, j \leq N$. 
Let $\rho_{2, Q}=\varepsilon_{Q}^{4}$ ( $\varepsilon_{Q}$ is specified in Assumption 2.3). Owing to Assumption 2.3, we have $\varepsilon_{Q}^{2} \leq r_{\theta}\left(x^{\prime} \mid y, x\right) \leq$ $1 / \varepsilon_{Q}^{2}$. Therefore, we get $A_{\theta}^{i, j}\left(v, v^{\prime}\right) \geq \varepsilon_{Q}^{4} / N=\rho_{2, Q} / N$. Hence, (i) is true.

Due to Assumptions 2.3 and 2.4, we have

$$
\begin{aligned}
\left|r_{\theta}\left(x^{\prime} \mid y, x\right)-r_{\theta^{\prime}}\left(x^{\prime} \mid y, x\right)\right| & \leq\left|p_{\theta}\left(x^{\prime} \mid x\right)-p_{\theta^{\prime}}\left(x^{\prime} \mid x\right)\right| q_{\theta}(y \mid x)+p_{\theta^{\prime}}\left(x^{\prime} \mid x\right)\left|q_{\theta}(y \mid x)-q_{\theta^{\prime}}(y \mid x)\right| \\
& \leq \frac{2 K_{1, Q}\left\|\theta-\theta^{\prime}\right\|}{\varepsilon_{Q}} .
\end{aligned}
$$

Then, we get

$$
\begin{aligned}
\left|A_{\theta}^{i, j}\left(v, v^{\prime}\right)-A_{\theta^{\prime}}^{i, j}\left(v, v^{\prime}\right)\right| & \leq \frac{\left|r_{\theta}\left(x_{j}^{\prime} \mid y, x_{i}\right)-r_{\theta^{\prime}}\left(x_{j}^{\prime} \mid y, x_{i}\right)\right|}{\sum_{k=1}^{N} r_{\theta}\left(x_{j}^{\prime} \mid y, x_{k}\right)}+A_{\theta^{\prime}}^{i, j}\left(v, v^{\prime}\right) \frac{\sum_{k=1}^{N}\left|r_{\theta}\left(x_{j}^{\prime} \mid y, x_{k}\right)-r_{\theta^{\prime}}\left(x_{j}^{\prime} \mid y, x_{k}\right)\right|}{\sum_{k=1}^{N} r_{\theta}\left(x_{j}^{\prime} \mid y, x_{k}\right)} \\
& \leq \frac{2 K_{1, Q}\left\|\theta-\theta^{\prime}\right\|}{\varepsilon_{Q}^{3}}\left(\frac{1}{N}+A_{\theta^{\prime}}^{i, j}\left(v, v^{\prime}\right)\right) .
\end{aligned}
$$

Since $\sum_{i=1}^{N} A_{\theta}^{i, j}\left(v, v^{\prime}\right)=1($ due to $(30)),(57)$ implies

$$
\sum_{i=1}^{N}\left|A_{\theta}^{i, j}\left(v, v^{\prime}\right)-A_{\theta^{\prime}}^{i, j}\left(v, v^{\prime}\right)\right| \leq \frac{2 K_{1, Q}\left\|\theta-\theta^{\prime}\right\|}{\varepsilon_{Q}^{3}}\left(1+\sum_{i=1}^{N} A_{\theta^{\prime}}^{i, j}\left(v, v^{\prime}\right)\right)=\frac{4 K_{1, Q}\left\|\theta-\theta^{\prime}\right\|}{\varepsilon_{Q}^{3}} .
$$

It is straightforward to verify

$$
B_{\theta}^{j}\left(v, v^{\prime}\right)=\sum_{i=1}^{N} A_{\theta}^{i, j}\left(v, v^{\prime}\right) \frac{\nabla_{\theta} r_{\theta}\left(x_{j}^{\prime} \mid y, x_{i}\right)}{r_{\theta}\left(x_{j}^{\prime} \mid y, x_{i}\right)} .
$$

Moreover, using Assumptions 2.3 and 2.4, we conclude

$$
\left\|\frac{\nabla_{\theta} r_{\theta}\left(x^{\prime} \mid y, x\right)}{r_{\theta}\left(x^{\prime} \mid y, x\right)}\right\| \leq\left\|\frac{\nabla_{\theta} p_{\theta}\left(x^{\prime} \mid x\right)}{p_{\theta}\left(x^{\prime} \mid x\right)}\right\|+\left\|\frac{\nabla_{\theta} q_{\theta}(y \mid x)}{q_{\theta}(y \mid x)}\right\| \leq \frac{2 K_{1, Q}}{\varepsilon_{Q}} .
$$

Relying on the same assumptions, we deduce

$$
\begin{aligned}
\left\|\frac{\nabla_{\theta} r_{\theta}\left(x^{\prime} \mid y, x\right)}{r_{\theta}\left(x^{\prime} \mid y, x\right)}-\frac{\nabla_{\theta} r_{\theta^{\prime}}\left(x^{\prime} \mid y, x\right)}{r_{\theta^{\prime}}\left(x^{\prime} \mid y, x\right)}\right\| \leq & \frac{\left\|\nabla_{\theta} p_{\theta}\left(x^{\prime} \mid x\right)-\nabla_{\theta} p_{\theta^{\prime}}\left(x^{\prime} \mid x\right)\right\|}{p_{\theta}\left(x^{\prime} \mid x\right)}+\left\|\frac{\nabla_{\theta} p_{\theta^{\prime}}\left(x^{\prime} \mid x\right)}{p_{\theta^{\prime}}\left(x^{\prime} \mid x\right)}\right\| \frac{\left|p_{\theta}\left(x^{\prime} \mid x\right)-p_{\theta^{\prime}}\left(x^{\prime} \mid x\right)\right|}{p_{\theta}\left(x^{\prime} \mid x\right)} \\
& +\frac{\left\|\nabla_{\theta} q_{\theta}(y \mid x)-\nabla_{\theta} q_{\theta^{\prime}}(y \mid x)\right\|}{q_{\theta}(y \mid x)}+\left\|\frac{\nabla_{\theta} q_{\theta^{\prime}}(y \mid x)}{q_{\theta^{\prime}}(y \mid x)}\right\| \frac{\left|q_{\theta}(y \mid x)-q_{\theta^{\prime}}(y \mid x)\right|}{q_{\theta}(y \mid x)} \\
\leq & \frac{4 K_{1, Q}^{2}\left\|\theta-\theta^{\prime}\right\|}{\varepsilon_{Q}^{2}} .
\end{aligned}
$$

Then, (30), (59), (60) imply

$$
\left\|B_{\theta}^{j}\left(v, v^{\prime}\right)\right\| \leq \sum_{i=1}^{N} A_{\theta}^{i, j}\left(v, v^{\prime}\right)\left\|\frac{\nabla_{\theta} r_{\theta}\left(x_{j}^{\prime} \mid y, x_{i}\right)}{r_{\theta}\left(x_{j}^{\prime} \mid y, x_{i}\right)}\right\| \leq \frac{2 K_{1, Q}}{\varepsilon_{Q}} .
$$

Similarly, (30), (58) - (61) yield

$$
\begin{aligned}
\left\|B_{\theta}^{j}\left(v, v^{\prime}\right)-B_{\theta^{\prime}}^{j}\left(v, v^{\prime}\right)\right\| \leq & \sum_{i=1}^{N}\left|A_{\theta}^{i, j}\left(v, v^{\prime}\right)-A_{\theta^{\prime}}^{i, j}\left(v, v^{\prime}\right)\right|\left\|\frac{\nabla_{\theta} r_{\theta}\left(x_{j}^{\prime} \mid y, x_{i}\right)}{r_{\theta}\left(x_{j}^{\prime} \mid y, x_{i}\right)}\right\| \\
& +\sum_{i=1}^{N} A_{\theta^{\prime}}^{i, j}\left(v, v^{\prime}\right)\left\|\frac{\nabla_{\theta} r_{\theta}\left(x_{j}^{\prime} \mid y, x_{i}\right)}{r_{\theta}\left(x_{j}^{\prime} \mid y, x_{i}\right)}-\frac{\nabla_{\theta} r_{\theta^{\prime}}\left(x_{j}^{\prime} \mid y, x_{i}\right)}{r_{\theta^{\prime}}\left(x_{j}^{\prime} \mid y, x_{i}\right)}\right\| \\
\leq & \frac{12 K_{1, Q}^{2}\left\|\theta-\theta^{\prime}\right\|}{\varepsilon_{Q}^{4}} .
\end{aligned}
$$


Due to Assumptions 2.3, 2.4, we have

$$
\left|C_{\theta}^{i}(v)\right| \leq \max \left\{\frac{1}{N}, \frac{q_{\theta}\left(y \mid x_{i}\right)}{\sum_{k=1}^{N} q_{\theta}\left(y \mid x_{k}\right)}\right\} \leq 1, \quad\left\|D_{\theta}(v)\right\| \leq \frac{\sum_{k=1}^{N}\left\|\nabla_{\theta} q_{\theta}\left(y \mid x_{k}\right)\right\|}{\sum_{k=1}^{N} q_{\theta}\left(y \mid x_{k}\right)} \leq \frac{K_{1, Q}}{\varepsilon_{Q}} .
$$

Combining Assumptions 2.3, 2.4 and the first part of (64), we get

$$
\begin{aligned}
\left|C_{\theta}^{i}(v)-C_{\theta^{\prime}}^{i}(v)\right| & \leq \frac{\left|q_{\theta}\left(y \mid x_{i}\right)-q_{\theta^{\prime}}\left(y \mid x_{i}\right)\right|}{\sum_{k=1}^{N} q_{\theta}\left(y \mid x_{k}\right)}+\frac{\left|C_{\theta^{\prime}}^{i}(v)\right| \sum_{k=1}^{N}\left|q_{\theta}\left(y \mid x_{k}\right)-q_{\theta^{\prime}}\left(y \mid x_{k}\right)\right|}{\sum_{k=1}^{N} q_{\theta}\left(y \mid x_{k}\right)} \\
& \leq \frac{2 K_{1, Q}\left\|\theta-\theta^{\prime}\right\|}{\varepsilon_{Q}} .
\end{aligned}
$$

Moreover, using Assumptions 2.3, 2.4 and the second part of (64), we get

$$
\begin{aligned}
\left\|D_{\theta}(v)-D_{\theta^{\prime}}(v)\right\| & \leq \frac{\sum_{k=1}^{N}\left\|\nabla_{\theta} q_{\theta}\left(y \mid x_{k}\right)-\nabla_{\theta} q_{\theta^{\prime}}\left(y \mid x_{k}\right)\right\|}{\sum_{k=1}^{N} q_{\theta}\left(y \mid x_{k}\right)}+\frac{\left\|D_{\theta^{\prime}}(v)\right\| \sum_{k=1}^{N}\left|q_{\theta}\left(y \mid x_{k}\right)-q_{\theta^{\prime}}\left(y \mid x_{k}\right)\right|}{\sum_{k=1}^{N} q_{\theta}\left(y \mid x_{k}\right)} \\
& \leq \frac{2 K_{1, Q}^{2}\left\|\theta-\theta^{\prime}\right\|}{\varepsilon_{Q}^{2}} .
\end{aligned}
$$

Let $C_{3, Q}=12 \varepsilon_{Q}^{-4} K_{1, Q}^{2} N$. Then, relying on (30), (58)- (66), we deduce that (54) - (56) hold. Hence, (ii) is true.

Lemma 5.3. Let Assumptions 2.3 and 2.4 hold. Then, there exist real numbers $\rho_{3, Q} \in(0,1), C_{4, Q} \in[1, \infty)$ (possibly depending on $N$ ) such that

$$
\begin{aligned}
& \max \left\{\left\|\Phi_{\theta}^{n}(v)\right\|,\left\|\Psi_{\theta}^{n}(v)\right\|\right\} \leq C_{4, Q} \rho_{3, Q}^{n}, \\
& \max \left\{\left\|\Phi_{\theta}^{n}(v)-\Phi_{\theta^{\prime}}^{n}(v)\right\|,\left\|\Psi_{\theta}^{n}(v)-\Phi_{\theta^{\prime}}^{n}(v)\right\|\right\} \leq C_{4, Q} \rho_{3, Q}^{n}\left\|\theta-\theta^{\prime}\right\|
\end{aligned}
$$

for all $\theta, \theta^{\prime} \in Q, v \in \mathcal{V}, n \geq 1$.

Proof. Throughout the proof, the following notation is used. $\theta, \theta^{\prime}$ are any elements of $Q$. $v$ is any element of $\mathcal{V}$, while $\left\{v_{n}\right\}_{n \geq 0}$ is any sequence in $\mathcal{V} . n$ is any positive integer.

Let $\rho_{3, Q}=\left(1-\rho_{2, Q}\right)^{1 / 2}, \tilde{C}_{1, Q}=4 \rho_{3, Q}^{-2} N, \tilde{C}_{2, Q}=2 \tilde{C}_{1, Q} C_{3, Q}^{2}, \tilde{C}_{3, Q}=\rho_{3, Q}^{-2} \tilde{C}_{2, Q} C_{3, Q}\left(\rho_{2, Q}, C_{3, Q}\right.$ are specified in Lemma 5.2). Owing to Lemmas 5.2, A1.2 (see Appendix) and (30), we have

$$
\left\|\tilde{A}_{\theta}^{n}\left(v_{0}, \ldots, v_{n}\right) C_{\theta}\left(v_{n}\right)\right\| \leq \tilde{C}_{1, Q} \rho_{3, Q}^{2 n}\left\|C_{\theta}\left(v_{n}\right)\right\| \leq \tilde{C}_{1, Q} C_{3, Q} \rho_{3, Q}^{2 n} \leq \tilde{C}_{2, Q} \rho_{3, Q}^{2 n}
$$

Since $A_{\theta}^{0}(v)=I$ (due to (39)), Lemma 5.2 and (67) imply

$$
\left\|\tilde{B}_{\theta}^{n}\left(v_{0}, \ldots, v_{n}\right) C_{\theta}\left(v_{n}\right)\right\| \leq\left\|B_{\theta}\left(v_{0}, v_{1}\right)\right\|\left\|\tilde{A}_{\theta}^{n-1}\left(v_{1}, \ldots, v_{n}\right) C_{\theta}\left(v_{n}\right)\right\| \leq \tilde{C}_{2, Q} C_{3, Q} \rho_{3, Q}^{2(n-1)}=\tilde{C}_{3, Q} \rho_{3, Q}^{2 n} .
$$

Moreover, due to Lemmas 5.2, A1.2 (see Appendix 1), we have

$$
\begin{aligned}
& \left\|\tilde{A}_{\theta}^{n}\left(v_{0}, \ldots, v_{n}\right) C_{\theta}\left(v_{n}\right)-\tilde{A}_{\theta^{\prime}}^{n}\left(v_{0}, \ldots, v_{n}\right) C_{\theta^{\prime}}\left(v_{n}\right)\right\| \\
& \leq \tilde{C}_{1, Q} \rho_{3, Q}^{2 n}\left(\left\|C_{\theta}\left(v_{n}\right)\right\|+\left\|C_{\theta^{\prime}}\left(v_{n}\right)\right\|\right) \sum_{k=0}^{n-1}\left\|A_{\theta}\left(v_{k}, v_{k+1}\right)-A_{\theta^{\prime}}\left(v_{k}, v_{k+1}\right)\right\| \\
& \quad+\tilde{C}_{1, Q} \rho_{3, Q}^{2 n}\left\|C_{\theta}\left(v_{n}\right)-C_{\theta^{\prime}}\left(v_{n}\right)\right\| \\
& \leq 2 \tilde{C}_{1, Q} C_{3, Q}^{2} \rho_{3, Q}^{2 n}(n+1)\left\|\theta-\theta^{\prime}\right\|=\tilde{C}_{2, Q} \rho_{3, Q}^{2 n}(n+1)\left\|\theta-\theta^{\prime}\right\| .
\end{aligned}
$$

Combining this with Lemma 5.2 and (67), we get

$$
\begin{aligned}
& \left\|\tilde{B}_{\theta}^{n}\left(v_{0}, \ldots, v_{n}\right) C_{\theta}\left(v_{n}\right)-\tilde{B}_{\theta^{\prime}}^{n}\left(v_{0}, \ldots, v_{n}\right) C_{\theta^{\prime}}\left(v_{n}\right)\right\| \\
& \leq\left\|B_{\theta^{\prime}}\left(v_{0}, v_{1}\right)\right\|\left\|\tilde{A}_{\theta}^{n-1}\left(v_{1}, \ldots, v_{n}\right) C_{\theta}\left(v_{n}\right)-\tilde{A}_{\theta^{\prime}}^{n-1}\left(v_{1}, \ldots, v_{n}\right) C_{\theta^{\prime}}\left(v_{n}\right)\right\| \\
& \quad+\left\|B_{\theta}\left(v_{0}, v_{1}\right)-B_{\theta^{\prime}}\left(v_{0}, v_{1}\right)\right\|\left\|\tilde{A}_{\theta}^{n-1}\left(v_{1}, \ldots, v_{n}\right) C_{\theta}\left(v_{n}\right)\right\| \\
& \leq \tilde{C}_{2, Q} C_{3, Q} \rho_{3, Q}^{2(n-1)}(n+1)\left\|\theta-\theta^{\prime}\right\|=\tilde{C}_{3, Q} \rho_{3, Q}^{2 n}(n+1)\left\|\theta-\theta^{\prime}\right\| .
\end{aligned}
$$


Let $U_{\theta}^{n}\left(d v_{1}, \ldots, d v_{n} \mid v\right)$ be the conditional probability measure defined for $B \in \mathcal{B}\left(\mathcal{V}^{n}\right)$ by

$$
U_{\theta}^{n}(B \mid v)=E\left(I_{B}\left(V_{1}^{\theta}, \ldots, V_{n}^{\theta}\right) \mid V_{0}^{\theta}=v\right) .
$$

Moreover, let $u_{\theta, \theta^{\prime}}^{n}(v)$ be the function defined by

$$
u_{\theta, \theta^{\prime}}^{n}(v)=\sup _{B \in \mathcal{B}\left(\mathcal{V}^{n}\right)}\left|U_{\theta}^{n}(B \mid v)-U_{\theta^{\prime}}^{n}(B \mid v)\right| .
$$

Then, for $B \in \mathcal{B}\left(\mathcal{V}^{n+1}\right)$, we have

$$
U_{\theta}^{n+1}(B \mid v)=\iint \cdots \int I_{B}\left(v_{1}, \ldots, v_{n}, v_{n+1}\right) T_{\theta}\left(v_{n}, d v_{n+1}\right) U_{\theta}^{n}\left(d v_{1}, \ldots, d v_{n} \mid v\right) .
$$

Consequently, Lemma 5.1 implies

$$
\begin{aligned}
\left|U_{\theta}^{n+1}(B \mid v)-U_{\theta^{\prime}}^{n+1}(B \mid v)\right| \leq & \iint \ldots \int I_{B}\left(v_{1}, \ldots, v_{n}, v_{n+1}\right)\left|T_{\theta}-T_{\theta^{\prime}}\right|\left(v_{n}, d v_{n+1}\right) U_{\theta}^{n}\left(d v_{1}, \ldots, d v_{n} \mid v\right) \\
& +\iint \ldots \int I_{B}\left(v_{1}, \ldots, v_{n}, v_{n+1}\right) T_{\theta^{\prime}}\left(v_{n}, d v_{n+1}\right)\left|U_{\theta}^{n}-U_{\theta^{\prime}}^{n}\right|\left(d v_{1}, \ldots, d v_{n} \mid v\right) \\
\leq & C_{2, Q}\left\|\theta-\theta^{\prime}\right\|+u_{\theta, \theta^{\prime}}^{n}(v)
\end{aligned}
$$

$\left(C_{2, Q}\right.$ is specified in Lemma 5.1). For $B \in \mathcal{B}(\mathcal{V})$, Lemma 5.1 also yields

$$
\left|U_{\theta}^{1}(B \mid v)-U_{\theta^{\prime}}^{1}(B \mid v)\right|=\left|T_{\theta}(v, B)-T_{\theta^{\prime}}(v, B)\right| \leq C_{2, Q}\left\|\theta-\theta^{\prime}\right\| .
$$

Hence, we have

$$
u_{\theta, \theta^{\prime}}^{1}(v) \leq C_{2, Q}\left\|\theta-\theta^{\prime}\right\|, \quad u_{\theta, \theta^{\prime}}^{n+1}(v) \leq u_{\theta, \theta^{\prime}}^{n}(v)+C_{2, Q}\left\|\theta-\theta^{\prime}\right\| .
$$

Then, iterating (71), we conclude

$$
u_{\theta, \theta^{\prime}}^{n}(v) \leq C_{2, Q} n\left\|\theta-\theta^{\prime}\right\| .
$$

Let $\tilde{C}_{4, Q} \in[1, \infty)$ be an upper bound of sequence $\left\{n \rho_{3, Q}^{n-1}\right\}_{n \geq 1}$, while $C_{4, Q}=4 \tilde{C}_{3, Q} \tilde{C}_{4, Q} C_{2, Q}$. It is straightforward to verify

$$
\begin{aligned}
\Phi_{\theta}^{n}(v) & =\int \cdots \int \tilde{A}_{\theta}^{n}\left(v, v_{1}, \ldots, v_{n}\right) C_{\theta}\left(v_{n}\right) U_{\theta}^{n}\left(d v_{1}, \ldots, d v_{n} \mid v\right), \\
\Psi_{\theta}^{n}(v) & =\int \cdots \int \tilde{B}_{\theta}^{n}\left(v, v_{1}, \ldots, v_{n}\right) C_{\theta}\left(v_{n}\right) U_{\theta}^{n}\left(d v_{1}, \ldots, d v_{n} \mid v\right) .
\end{aligned}
$$

Combining this with (67), (68), we get

$$
\begin{aligned}
& \left\|\Phi_{\theta}^{n}(v)\right\| \leq \int \cdots \int\left\|\tilde{A}_{\theta}^{n}\left(v, v_{1}, \ldots, v_{n}\right) C_{\theta}\left(v_{n}\right)\right\| U_{\theta}^{n}\left(d v_{1}, \ldots, d v_{n} \mid v\right) \leq \tilde{C}_{2, Q} \rho_{3, Q}^{2 n} \leq C_{4, Q} \rho_{3, Q}^{n}, \\
& \left\|\Psi_{\theta}^{n}(v)\right\| \leq \int \cdots \int\left\|\tilde{B}_{\theta}^{n}\left(v, v_{1}, \ldots, v_{n}\right) C_{\theta}\left(v_{n}\right)\right\| U_{\theta}^{n}\left(d v_{1}, \ldots, d v_{n} \mid v\right) \leq \tilde{C}_{3, Q} \rho_{3, Q}^{2 n} \leq C_{4, Q} \rho_{3, Q}^{n} .
\end{aligned}
$$

Moreover, (69), (73), (72) imply

$$
\begin{aligned}
\left\|\Phi_{\theta}^{n}(v)-\Phi_{\theta^{\prime}}^{n}(v)\right\| \leq & \int \ldots \int\left\|\tilde{A}_{\theta}^{n}\left(v, v_{1}, \ldots, v_{n}\right) C_{\theta}\left(v_{n}\right)-\tilde{A}_{\theta^{\prime}}^{n}\left(v, v_{1}, \ldots, v_{n}\right) C_{\theta^{\prime}}\left(v_{n}\right)\right\| U_{\theta}^{n}\left(d v_{1}, \ldots, d v_{n} \mid v\right) \\
& +\int \cdots \int\left\|\tilde{A}_{\theta^{\prime}}^{n}\left(v, v_{1}, \ldots, v_{n}\right) C_{\theta^{\prime}}\left(v_{n}\right)\right\|\left|U_{\theta}^{n}-U_{\theta^{\prime}}^{n}\right|\left(d v_{1}, \ldots, d v_{n} \mid v\right) \\
\leq & 2 \tilde{C}_{2, Q} C_{2, Q} \rho_{3, Q}^{2 n}(n+1)\left\|\theta-\theta^{\prime}\right\| \leq C_{4, Q} \rho_{3, Q}^{n}\left\|\theta-\theta^{\prime}\right\| .
\end{aligned}
$$


Similarly, (70), (74), (72) yield

$$
\begin{aligned}
\left\|\Psi_{\theta}^{n}(v)-\Psi_{\theta^{\prime}}^{n}(v)\right\| \leq & \int \ldots \int\left\|\tilde{B}_{\theta}^{n}\left(v, v_{1}, \ldots, v_{n}\right) C_{\theta}\left(v_{n}\right)-\tilde{B}_{\theta^{\prime}}^{n}\left(v, v_{1}, \ldots, v_{n}\right) C_{\theta^{\prime}}\left(v_{n}\right)\right\| U_{\theta}^{n}\left(d v_{1}, \ldots, d v_{n} \mid v\right) \\
& +\int \cdots \int\left\|\tilde{B}_{\theta^{\prime}}^{n}\left(v, v_{1}, \ldots, v_{n}\right) C_{\theta^{\prime}}\left(v_{n}\right)\right\|\left|U_{\theta}^{n}-U_{\theta^{\prime}}^{n}\right|\left(d v_{1}, \ldots, d v_{n} \mid v\right) \\
\leq & 2 \tilde{C}_{3, Q} C_{2, Q} \rho_{3, Q}^{2 n}(n+1)\left\|\theta-\theta^{\prime}\right\| \leq C_{4, Q} \rho_{3, Q}^{n}\left\|\theta-\theta^{\prime}\right\| .
\end{aligned}
$$

Lemma 5.4. Let Assumptions 2.2 - 2.4 hold. Then, the following is true:

(i) $h(\theta)$ is well-defined on $\Theta$.

(ii) $h(\theta)=\lim _{n \rightarrow \infty} E\left(H\left(\theta, Z_{n}^{\theta}\right)\right)$ for each $\theta \in \Theta$ satisfying $E\left(\left\|W_{0}^{\theta} \Lambda\right\|\right)<\infty$.

(iii) There exists a function $\tilde{H}(\theta, z)$ mapping $\theta \in \Theta, z \in \mathcal{Z}$ to $\mathbb{R}^{d}$ such that

$$
H(\theta, z)-h(\theta)=\tilde{H}(\theta, z)-(\Pi \tilde{H})(\theta, z)
$$

for all $\theta \in \Theta, z \in \mathcal{Z}$. Here, $(\Pi \tilde{H})(\theta, z)$ denotes $\int \tilde{H}\left(\theta, z^{\prime}\right) \Pi_{\theta}\left(z, d z^{\prime}\right)$.

(iv) There exists a real number $C_{5, Q} \in[1, \infty)$ (possibly depending on $N$ ) such that

$$
\begin{aligned}
& \max \{\|H(\theta, z)\|,\|\tilde{H}(\theta, z)\|,\|(\Pi \tilde{H})(\theta, z)\|\} \leq C_{5, Q}(1+\|W \Lambda\|), \\
& \left\|(\Pi \tilde{H})(\theta, z)-(\Pi \tilde{H})\left(\theta^{\prime}, z\right)\right\| \leq C_{5, Q}\left\|\theta-\theta^{\prime}\right\|(1+\|W \Lambda\|)
\end{aligned}
$$

for all $\theta, \theta^{\prime} \in Q, v \in \mathcal{V}, W \in \mathbb{R}^{d \times N}$ and $z=(v, W$ ) ( $\Lambda$ is defined in (26)).

Proof. Throughout the proof, the following notation is used. $\theta, \theta^{\prime}$ are any elements of $Q . v, W$ are any elements of $\mathcal{V}, \mathbb{R}^{d \times N}$ (respectively), while $z=(v, W) . n, k$ are any positive integers.

Owing to (30), we have

$$
e^{T} \tilde{A}_{\theta}^{n}\left(V_{0}^{\theta}, \ldots, V_{n}^{\theta}\right) C_{\theta}\left(V_{n}^{\theta}\right)=e^{T} C_{\theta}\left(V_{n}^{\theta}\right)=0 .
$$

Therefore, we get

$$
\begin{aligned}
\Lambda \tilde{A}_{\theta}^{n}\left(V_{0}^{\theta}, \ldots, V_{n}^{\theta}\right) C_{\theta}\left(V_{n}^{\theta}\right) & =\tilde{A}_{\theta}^{n}\left(V_{0}^{\theta}, \ldots, V_{n}^{\theta}\right) C_{\theta}\left(V_{n}^{\theta}\right)-\frac{e}{N} e^{T} \tilde{A}_{\theta}^{n}\left(V_{0}^{\theta}, \ldots, V_{n}^{\theta}\right) C_{\theta}\left(V_{n}^{\theta}\right) \\
& =\tilde{A}_{\theta}^{n}\left(V_{0}^{\theta}, \ldots, V_{n}^{\theta}\right) C_{\theta}\left(V_{n}^{\theta}\right) .
\end{aligned}
$$

Moreover, iterating (37), it is straightforward to verify

$$
W_{n}^{\theta}=W_{0}^{\theta} \tilde{A}_{\theta}^{n}\left(V_{0}^{\theta}, \ldots, V_{n}^{\theta}\right)+\sum_{k=0}^{n-1} \tilde{B}_{\theta}^{n-k}\left(V_{k}^{\theta}, \ldots, V_{n}^{\theta}\right) .
$$

Combining this with (76), we conclude

$$
\begin{aligned}
H\left(\theta, Z_{n}^{\theta}\right) & =D_{\theta}\left(V_{n}^{\theta}\right)+W_{0}^{\theta} \tilde{A}_{\theta}^{n}\left(V_{0}^{\theta}, \ldots, V_{n}^{\theta}\right) C_{\theta}\left(V_{n}^{\theta}\right)+\sum_{k=0}^{n-1} \tilde{B}_{\theta}^{n-k}\left(V_{k}^{\theta}, \ldots, V_{n}^{\theta}\right) C_{\theta}\left(V_{n}^{\theta}\right) \\
& =D_{\theta}\left(V_{n}^{\theta}\right)+W_{0}^{\theta} \Lambda \tilde{A}_{\theta}^{n}\left(V_{0}^{\theta}, \ldots, V_{n}^{\theta}\right) C_{\theta}\left(V_{n}^{\theta}\right)+\sum_{k=0}^{n-1} \tilde{B}_{\theta}^{n-k}\left(V_{k}^{\theta}, \ldots, V_{n}^{\theta}\right) C_{\theta}\left(V_{n}^{\theta}\right) .
\end{aligned}
$$

Consequently, we have

$$
\begin{aligned}
\left(\Pi^{n} H\right)(\theta, z)=E\left(H\left(\theta, Z_{n}^{\theta}\right) \mid Z_{0}^{\theta}=z\right)= & E\left(D_{\theta}\left(V_{n}^{\theta}\right) \mid V_{0}^{\theta}=v\right)+E\left(W \Lambda \tilde{A}_{\theta}^{n}\left(V_{0}^{\theta}, \ldots, V_{n}^{\theta}\right) C_{\theta}\left(V_{n}^{\theta}\right) \mid V_{0}^{\theta}=v\right) \\
& +\sum_{k=0}^{n-1} E\left(E\left(\tilde{B}_{\theta}^{n-k}\left(V_{k}^{\theta}, \ldots, V_{n}^{\theta}\right) C_{\theta}\left(V_{n}^{\theta}\right) \mid V_{k}^{\theta}\right) \mid V_{0}^{\theta}=v\right) \\
= & \left(T^{n} D\right)_{\theta}(v)+W \Lambda \Phi_{\theta}^{n}(v)+\sum_{k=0}^{n-1} E\left(\Psi_{\theta}^{n-k}\left(V_{k}^{\theta}\right) \mid V_{0}^{\theta}=v\right) \\
= & \left(T^{n} D\right)_{\theta}(v)+W \Lambda \Phi_{\theta}^{n}(v)+\sum_{k=0}^{n-1}\left(T^{k} \Psi^{n-k}\right)_{\theta}(v) .
\end{aligned}
$$


Here, $\left(\Pi^{n} H\right)(\theta, z)$ denotes $\int H\left(\theta, z^{\prime}\right) \Pi_{\theta}^{n}\left(z, d z^{\prime}\right)$, while $\left(T^{n} D\right)_{\theta}(v),\left(T^{k} \Psi^{l}\right)_{\theta}(v)$ stand for $\int D_{\theta}\left(v^{\prime}\right) T_{\theta}^{n}\left(v, d v^{\prime}\right)$, $\int \Psi_{\theta}^{l}\left(v^{\prime}\right) T_{\theta}^{k}\left(v, d v^{\prime}\right)$ (respectively).

Let $\beta_{Q}=\max \left\{\rho_{1, Q}^{1 / 2}, \rho_{3, Q}^{1 / 2}\right\}, \tilde{C}_{1, Q}=4 C_{2, Q} C_{3, Q} C_{4, Q}, \tilde{C}_{2, Q}=2 \tilde{C}_{1, Q}\left(1-\beta_{Q}\right)^{-1}\left(\rho_{1, Q}, \rho_{3, Q}, C_{2, Q}, C_{3, Q}\right.$, $C_{4, Q}$ are specified in Lemmas $\left.5.1-5.3\right)$. Owing to Lemma 5.3, we have

$$
\int\left\|\Psi_{\theta}^{n}(v)\right\| \tau_{\theta}(d v) \leq C_{4, Q} \rho_{3, Q}^{n} \leq \tilde{C}_{1, Q} \beta_{Q}^{2 n} .
$$

Consequently, Lemma 5.2 yields

$$
\int\left\|D_{\theta}(v)\right\| \tau_{\theta}(d v)+\sum_{n=1}^{\infty} \int\left\|\Psi_{\theta}^{n}(v)\right\| \tau_{\theta}(d v) \leq C_{3, Q}+\tilde{C}_{1, Q} \sum_{n=1}^{\infty} \beta_{Q}^{2 n} \leq 2 \tilde{C}_{1, Q}\left(1-\beta_{Q}\right)^{-1} \leq \tilde{C}_{2, Q}<\infty .
$$

Hence, $h(\theta)$ is well-defined and satisfies $\|h(\theta)\| \leq \tilde{C}_{2, Q}$. Since $Q$ is any compact set in $\Theta$, we conclude that (i) holds. Moreover, using (78), we deduce

$$
\left(\Pi^{n} H\right)(\theta, z)-h(\theta)=\left(\tilde{T}^{n} D\right)_{\theta}(v)+W \Lambda \Phi_{\theta}^{n}(v)+\sum_{k=0}^{n-1}\left(\tilde{T}^{k} \Psi^{n-k}\right)_{\theta}(v)-\sum_{k=n+1}^{\infty} \int \Psi_{\theta}^{k}\left(v^{\prime}\right) \tau_{\theta}\left(d v^{\prime}\right) .
$$

Here, $\left(\tilde{T}^{n} D\right)_{\theta}(v),\left(\tilde{T}^{k} \Psi^{l}\right)_{\theta}(v)$ denote $\int D_{\theta}\left(v^{\prime}\right) \tilde{T}_{\theta}^{n}\left(v, d v^{\prime}\right), \int \Psi_{\theta}^{l}\left(v^{\prime}\right) \tilde{T}_{\theta}^{k}\left(v, d v^{\prime}\right)$ (respectively).

Let $\tilde{C}_{3, Q} \in[1, \infty)$ be an upper bound of sequence $\left\{n \beta_{Q}^{n-1}\right\}_{n \geq 1}$, while $\tilde{C}_{4, Q}=2 \tilde{C}_{2, Q} \tilde{C}_{3, Q}\left(1-\beta_{Q}\right)^{-1}$, $C_{5, Q}=\tilde{C}_{4, Q}\left(1-\beta_{Q}\right)^{-1}$. Owing to Lemmas 5.1, 5.2, we have

$$
\left\|\left(\tilde{T}^{n} D\right)_{\theta}(v)\right\| \leq \int\left\|D_{\theta}\left(v^{\prime}\right)\right\|\left|\tilde{T}_{\theta}^{n}\right|\left(v, d v^{\prime}\right) \leq C_{2, Q} C_{3, Q} \rho_{1, Q}^{n} \leq \tilde{C}_{1, Q} \beta_{Q}^{2 n} .
$$

Similarly, due to Lemmas 5.1, 5.3, we have

$$
\left\|\left(\tilde{T}^{n-k} \Psi^{k}\right)_{\theta}(v)\right\| \leq \int\left\|\Psi_{\theta}^{k}\left(v^{\prime}\right)\right\|\left|\tilde{T}_{\theta}^{n-k}\right|\left(v, d v^{\prime}\right) \leq C_{2, Q} C_{4, Q} \rho_{1, Q}^{n-k} \rho_{3, Q}^{k} \leq \tilde{C}_{1, Q} \beta_{Q}^{2 n} .
$$

Combining Lemma 5.3 and (79), (80) - (82), we get

$$
\begin{aligned}
\left\|\left(\Pi^{n} H\right)(\theta, z)-h(\theta)\right\| & \leq\left\|\left(\tilde{T}^{n} D\right)_{\theta}(v)\right\|+\left\|\Phi_{\theta}^{n}(v)\right\|\|W \Lambda\|+\sum_{k=1}^{n}\left\|\left(\tilde{T}^{n-k} \Psi^{k}\right)_{\theta}(v)\right\|+\sum_{k=n+1}^{\infty}\left\|\Psi_{\theta}^{k}\left(v^{\prime}\right)\right\| \tau_{\theta}\left(d v^{\prime}\right) \\
& \leq \tilde{C}_{1, Q} \beta_{Q}^{2 n}(n+1)+C_{4, Q} \rho_{3, Q}^{n}\|W \Lambda\|+\tilde{C}_{1, Q} \sum_{k=n+1}^{\infty} \beta_{Q}^{2 k} \\
& \leq \tilde{C}_{1, Q} \beta_{Q}^{2 n}(n+1)(1+\|W \Lambda\|)+\tilde{C}_{1, Q} \beta_{Q}^{2 n}\left(1-\beta_{Q}\right)^{-1} \leq \tilde{C}_{4, Q} \beta_{Q}^{n}(1+\|W \Lambda\|) .
\end{aligned}
$$

Since $\|h(\theta)\| \leq \tilde{C}_{2, Q}$ and $\Lambda C_{\theta}(v)=C_{\theta}(v)$ (due to (30)), Lemma 5.2 yields

$$
\begin{aligned}
\left\|\left(\Pi^{0} H\right)(\theta, z)-h(\theta)\right\| \leq\left\|C_{\theta}(v)\right\|\|W \Lambda\|+\left\|D_{\theta}(v)\right\|+\|h(\theta)\| & \leq C_{3, Q}(1+\|W \Lambda\|)+\tilde{C}_{2, Q} \\
& \leq \tilde{C}_{4, Q}(1+\|W \Lambda\|) .
\end{aligned}
$$

Hence, we have

$$
\sum_{n=0}^{\infty}\left\|\left(\Pi^{n} H\right)(\theta, z)-h(\theta)\right\| \leq \tilde{C}_{4, Q}(1+\|W \Lambda\|) \sum_{n=0}^{\infty} \beta_{Q}^{n} \leq C_{5, Q}(1+\|W \Lambda\|) .
$$

Owing to Lemmas 5.1, 5.2, we have

$$
\begin{aligned}
\left\|\left(\tilde{T}^{n} D\right)_{\theta}(v)-\left(\tilde{T}^{n} D\right)_{\theta^{\prime}}(v)\right\| & \leq \int\left\|D_{\theta}\left(v^{\prime}\right)-D_{\theta^{\prime}}\left(v^{\prime}\right)\right\|\left|\tilde{T}_{\theta}^{n}\right|\left(v, d v^{\prime}\right)+\int\left\|D_{\theta^{\prime}}\left(v^{\prime}\right)\right\| \tilde{T}_{\theta}^{n}-\tilde{T}_{\theta^{\prime}}^{n} \mid\left(v, d v^{\prime}\right) \\
& \leq 2 C_{2, Q} C_{3, Q} \rho_{1, Q}^{n}\left\|\theta-\theta^{\prime}\right\| \leq \tilde{C}_{1, Q} \beta_{Q}^{2 n}\left\|\theta-\theta^{\prime}\right\| .
\end{aligned}
$$


Moreover, Lemmas 5.1, 5.3 imply

$$
\begin{aligned}
\left\|\left(\tilde{T}^{n-k} \Psi^{k}\right)_{\theta}(v)-\left(\tilde{T}^{n-k} \Psi^{k}\right)_{\theta^{\prime}}(v)\right\| \leq & \int\left\|\Psi_{\theta}^{k}\left(v^{\prime}\right)-\Psi_{\theta^{\prime}}^{k}\left(v^{\prime}\right)\right\|\left|\tilde{T}_{\theta}^{n-k}\right|\left(v, d v^{\prime}\right) \\
& +\int\left\|\Psi_{\theta^{\prime}}^{k}\left(v^{\prime}\right)\right\| \tilde{T}_{\theta}^{n-k}-\tilde{T}_{\theta^{\prime}}^{n-k} \mid\left(v, d v^{\prime}\right) \\
\leq & 2 C_{2, Q} C_{4, Q} \rho_{1, Q}^{n-k} \rho_{3, Q}^{k}\left\|\theta-\theta^{\prime}\right\| \leq \tilde{C}_{1, Q} \beta_{Q}^{2 n}\left\|\theta-\theta^{\prime}\right\|
\end{aligned}
$$

for $n \geq k$. The same lemmas also yield

$$
\begin{aligned}
\left\|\int \Psi_{\theta}^{n}(v) \tau_{\theta}(d v)-\int \Psi_{\theta^{\prime}}^{n}(v) \tau_{\theta^{\prime}}(d v)\right\| & \leq \int\left\|\Psi_{\theta}^{n}(v)-\Psi_{\theta^{\prime}}^{n}(v)\right\| \tau_{\theta}(d v)+\int\left\|\Psi_{\theta^{\prime}}^{n}(v)\right\| \tau_{\theta}-\tau_{\theta^{\prime}} \mid(d v) \\
& \leq 2 C_{2, Q} C_{4, Q} \rho_{3, Q}^{n}\left\|\theta-\theta^{\prime}\right\| \leq \tilde{C}_{1, Q} \beta_{Q}^{2 n}\left\|\theta-\theta^{\prime}\right\| .
\end{aligned}
$$

Combining Lemma 5.3 and (80), (85) - (87), we get

$$
\begin{aligned}
& \left\|\left(\left(\Pi^{n} H\right)(\theta, z)-h(\theta)\right)-\left(\left(\Pi^{n} H\right)\left(\theta^{\prime}, z\right)-h\left(\theta^{\prime}\right)\right)\right\| \\
& \leq\left\|\left(\tilde{T}^{n} D\right)_{\theta}(v)-\left(\tilde{T}^{n} D\right)_{\theta^{\prime}}(v)\right\|+\left\|\Phi_{\theta}^{n}(v)-\Phi_{\theta^{\prime}}^{n}(v)\right\|\|W \Lambda\| \\
& \quad+\sum_{k=1}^{n}\left\|\left(\tilde{T}^{n-k} \Psi^{k}\right)_{\theta}(v)-\left(\tilde{T}^{n-k} \Psi^{k}\right)_{\theta^{\prime}}(v)\right\|+\sum_{k=n+1}^{\infty}\left\|\int \Psi_{\theta}^{k}\left(v^{\prime}\right) \tau_{\theta}\left(d v^{\prime}\right)-\int \Psi_{\theta^{\prime}}^{k}\left(v^{\prime}\right) \tau_{\theta^{\prime}}\left(d v^{\prime}\right)\right\| \\
& \leq \tilde{C}_{1, Q} \beta_{Q}^{2 n}(n+1)\left\|\theta-\theta^{\prime}\right\|+C_{4, Q} \rho_{3, Q}^{n}\|W \Lambda\|\left\|\theta-\theta^{\prime}\right\|+\tilde{C}_{1, Q}\left\|\theta-\theta^{\prime}\right\| \sum_{k=n+1}^{\infty} \beta_{Q}^{2 k} \\
& \leq \tilde{C}_{1, Q} \beta_{Q}^{2 n}(n+1)(1+\|W \Lambda\|)\left\|\theta-\theta^{\prime}\right\|+\tilde{C}_{1, Q} \beta_{Q}^{2 n}\left(1-\beta_{Q}\right)^{-1}\left\|\theta-\theta^{\prime}\right\| \\
& \leq \tilde{C}_{4, Q} \beta_{Q}^{n}\left\|\theta-\theta^{\prime}\right\|(1+\|W \Lambda\|) .
\end{aligned}
$$

Hence, we have

$$
\begin{aligned}
\sum_{n=1}^{\infty}\left\|\left(\left(\Pi^{n} H\right)(\theta, z)-h(\theta)\right)-\left(\left(\Pi^{n} H\right)\left(\theta^{\prime}, z\right)-h\left(\theta^{\prime}\right)\right)\right\| & \leq \tilde{C}_{4, Q}\left\|\theta-\theta^{\prime}\right\|(1+\|W \Lambda\|) \sum_{n=1}^{\infty} \beta_{Q}^{n} \\
& \leq C_{5, Q}\left\|\theta-\theta^{\prime}\right\|(1+\|W \Lambda\|) .
\end{aligned}
$$

Let $\tilde{H}(\theta, z)$ be the function defined by

$$
\tilde{H}(\theta, z)=\sum_{n=0}^{\infty}\left(\left(\Pi^{n} H\right)(\theta, z)-h(\theta)\right) .
$$

Then, (84) implies that $\tilde{H}(\theta, z),(\Pi \tilde{H})(\theta, z)$ are well-defined and satisfy

$$
(\Pi \tilde{H})(\theta, z)=\sum_{n=1}^{\infty}\left(\left(\Pi^{n} H\right)(\theta, z)-h(\theta)\right) .
$$

Consequently, (75) holds. Since $Q$ is any compact set in $\Theta$, we conclude that (iii) also holds. Moreover, using (84), (89), we deduce that (iv) is also true.

When $E\left(\left\|W_{0}^{\theta} \Lambda\right\|\right)<\infty$, (83) implies

$$
\begin{aligned}
\left\|E\left(H\left(\theta, Z_{n}^{\theta}\right)\right)-h(\theta)\right\|=\left\|E\left(\left(\Pi^{n} H\right)\left(\theta, Z_{0}^{\theta}\right)-h(\theta)\right)\right\| & \leq E\left(\left\|\left(\Pi^{n} H\right)\left(\theta, Z_{0}^{\theta}\right)-h(\theta)\right\|\right) \\
& \leq \tilde{C}_{4, Q} \beta_{Q}^{n}\left(1+E\left(\left\|W_{0}^{\theta} \Lambda\right\|\right)\right) .
\end{aligned}
$$

Therefore, $h(\theta)=\lim _{n \rightarrow \infty} E\left(H\left(\theta, Z_{n}^{\theta}\right)\right)$ if $E\left(\left\|W_{0}^{\theta} \Lambda\right\|\right)<\infty$. As $Q$ is any compact set in $\Theta$, we conclude that (ii) holds. 
Lemma 5.5. Let Assumptions 2.3 and 2.4 hold. Then, there exists a real number $C_{6, Q} \in[1, \infty$ ) (possibly depending on $N$ ) such that

$$
\left\|W_{n} \Lambda\right\| I_{\left\{\tau_{Q} \geq n\right\}} \leq C_{6, Q}\left(1+\left\|W_{0} \Lambda\right\|\right)
$$

for $n \geq 1$, where $\tau_{Q}$ is the stopping time defined by

$$
\tau_{Q}=\inf \left(\left\{n \geq 0: \theta_{n} \notin Q\right\} \cup\{\infty\}\right)
$$

( $\Lambda$ is specified in (26)).

Proof. Throughout the proof, the following notation is used. $n$ is any positive integer. $A_{n}$ and $B_{n}$ are the random matrices defined by

$$
A_{n}=A_{\theta_{n-1}}\left(V_{n-1}, V_{n}\right), \quad B_{n}=B_{\theta_{n-1}}\left(V_{n-1}, V_{n}\right) .
$$

$A_{k, k}$ and $A_{k, l}$ are the random matrices defined by

$$
A_{k, k}=I, \quad A_{k, l}=A_{k+1} \cdots A_{l}
$$

for $l>k \geq 0$. Then, iterating (13), we get

$$
W_{n}=W_{0} A_{0, n}+\sum_{j=1}^{n} B_{j} A_{j, n} .
$$

Moreover, (30) implies $e^{T} A_{k, l} \Lambda=e^{T} \Lambda=0$ for $l \geq k \geq 0$. Consequently, we have

$$
\Lambda A_{k, l} \Lambda=A_{k, l} \Lambda-\frac{e}{N} e^{T} A_{k, l} \Lambda=A_{k, l} \Lambda .
$$

Combining this with (90), we get

$$
W_{n} \Lambda=W_{0} A_{0, n} \Lambda+\sum_{j=1}^{n} B_{j} A_{j, n} \Lambda=W_{0} \Lambda A_{0, n} \Lambda+\sum_{j=1}^{n} B_{k} A_{j, n} \Lambda .
$$

Let $\beta_{Q}=1-\rho_{2, Q}, \tilde{C}_{1, Q}=4 \beta_{Q}^{-1} N, \tilde{C}_{2, Q}=\tilde{C}_{1, Q} C_{3, Q}, C_{6, Q}=\tilde{C}_{2, Q}\left(1-\beta_{Q}\right)^{-1}\left(\rho_{2, Q}, C_{3, Q}\right.$ are specified in Lemma 5.2). Since $\theta_{0}, \ldots, \theta_{n-1} \in Q$ on $\left\{\tau_{Q} \geq n\right\}$, Lemmas 5.2, A1.2 (see Appendix 1) and (30) imply

$$
\left\|A_{k, n} \Lambda\right\| I_{\left\{\tau_{Q} \geq n\right\}}=\left\|A_{\theta_{k}}\left(V_{k}, V_{k+1}\right) \cdots A_{\theta_{n-1}}\left(V_{n-1}, V_{n}\right) \Lambda\right\| I_{\left\{\tau_{Q} \geq n\right\}} \leq \tilde{C}_{1, Q} \beta_{Q}^{n-k}
$$

for $n>k \geq 0$. Consequently, Lemma 5.2 yields

$$
\begin{aligned}
\left\|B_{k} A_{k, n} \Lambda\right\| I_{\left\{\tau_{Q} \geq n\right\}}=\left\|B_{\theta_{k-1}}\left(V_{k-1}, V_{k}\right) A_{k, n} \Lambda\right\| I_{\left\{\tau_{Q} \geq n\right\}} & \leq\left\|B_{\theta_{k-1}}\left(V_{k-1}, V_{k}\right)\right\|\left\|A_{k, n} \Lambda\right\| I_{\left\{\tau_{Q} \geq n\right\}} \\
& \leq \tilde{C}_{1, Q} C_{3, Q} \beta_{Q}^{n-k} \leq \tilde{C}_{2, Q} \beta_{Q}^{n-k}
\end{aligned}
$$

for $n \geq k \geq 1$. Combining this with (91), we get

$$
\begin{aligned}
\left\|W_{n} \Lambda\right\| I_{\left\{\tau_{Q} \geq n\right\}} & \leq\left\|W_{0} \Lambda\right\|\left\|A_{0, n} \Lambda\right\| I_{\left\{\tau_{Q} \geq n\right\}}+\sum_{j=1}^{n}\left\|B_{j} A_{j, n} \Lambda\right\| I_{\left\{\tau_{Q} \geq n\right\}} \\
& \leq \tilde{C}_{1, Q} \beta_{Q}^{n}\left\|W_{0} \Lambda\right\|+\tilde{C}_{2, Q} \sum_{j=1}^{n} \beta_{Q}^{n-j} \\
& \leq \tilde{C}_{2, Q}\left(1-\beta_{Q}\right)^{-1}\left(1+\left\|W_{0} \Lambda\right\|\right)=C_{6, Q}\left(1+\left\|W_{0} \Lambda\right\|\right) .
\end{aligned}
$$


Lemma 5.6. Let Assumptions 2.2-2.4 hold. Then, there exists a real number $M_{Q} \in[1, \infty)$ (independent of $N$ and depending only on $\left.p_{\theta}\left(x^{\prime} \mid x\right), q_{\theta}(y \mid x)\right)$ such that

$$
\|h(\theta)-\nabla l(\theta)\| \leq \frac{M_{Q}}{N}
$$

for all $\theta \in Q$.

Proof. Throughout the proof, the following notation is used. $H_{\theta, y}^{\prime}(\xi, \zeta)$ and $H_{\theta, y}^{\prime \prime}(\xi, \zeta)$ are the functions defined by

$$
H_{\theta, y}^{\prime}(\xi, \zeta)=\frac{\int \nabla_{\theta} q_{\theta}(y \mid x) \xi(d x)}{\int q_{\theta}(y \mid x) \xi(d x)}, \quad H_{\theta, y}^{\prime \prime}(\xi, \zeta)=\frac{\int q_{\theta}(y \mid x) \zeta(d x)}{\int q_{\theta}(y \mid x) \xi(d x)}
$$

for $\theta \in \Theta, y \in \mathcal{Y}, \xi \in \mathcal{P}(\mathcal{X}), \zeta \in \mathcal{M}_{s}^{d}(\mathcal{X}) . A_{1, n}^{\theta}, A_{2, n}^{\theta}$ and $A_{3, n}^{\theta}$ are the random variables defined by

$$
A_{1, n}^{\theta}=\int q_{\theta}\left(Y_{n} \mid x\right) \xi_{n}^{\theta}(d x), \quad A_{2, n}^{\theta}=\int q_{\theta}\left(Y_{n} \mid x\right) \tilde{\xi}_{n}^{\theta}(d x), \quad A_{3, n}^{\theta}=\int q_{\theta}\left(Y_{n} \mid x\right) F_{\theta, \boldsymbol{Y}}^{0: n}\left(d x \mid \xi_{0}^{\theta}\right)
$$

for $n \geq 0 . B_{1, n}^{\theta}, B_{2, n}^{\theta}$ and $B_{3, n}^{\theta}$ are the random variables defined by

$$
B_{1, n}^{\theta}=\int \nabla_{\theta} q_{\theta}\left(Y_{n} \mid x\right) \xi_{n}^{\theta}(d x), \quad B_{2, n}^{\theta}=\int \nabla_{\theta} q_{\theta}\left(Y_{n} \mid x\right) \tilde{\xi}_{n}^{\theta}(d x), \quad B_{3, n}^{\theta}=\int \nabla_{\theta} q_{\theta}\left(Y_{n} \mid x\right) F_{\theta, \boldsymbol{Y}}^{0: n}\left(d x \mid \xi_{0}^{\theta}\right) .
$$

$C_{1, n}^{\theta}, C_{2, n}^{\theta}$ and $C_{3, n}^{\theta}$ are the random variables defined by

$$
C_{1, n}^{\theta}=\int q_{\theta}\left(Y_{n} \mid x\right) \zeta_{n}^{\theta}(d x), \quad C_{2, n}^{\theta}=\int q_{\theta}\left(Y_{n} \mid x\right) \tilde{\zeta}_{n}^{\theta}(d x), \quad C_{3, n}^{\theta}=\int q_{\theta}\left(Y_{n} \mid x\right) G_{\theta, \boldsymbol{Y}}^{0: n}\left(d x \mid \xi_{0}^{\theta}, \zeta_{0}\right) .
$$

For $1 \leq i \leq N, W_{n, i}^{\theta}$ is the $i$-th column of $W_{n}^{\theta} \cdot \xi_{n}^{\theta}(d x)$ and $\zeta_{n}^{\theta}(d x)$ are the (empirical) measures defined by

$$
\xi_{n}^{\theta}(B)=\frac{1}{N} \sum_{i=1}^{N} \delta_{\hat{X}_{n, i}^{\theta}}(B), \quad \zeta_{n}^{\theta}(B)=\frac{1}{N} \sum_{i=1}^{N}\left(W_{n, i}^{\theta}-\frac{1}{N} \sum_{j=1}^{N} W_{n, j}^{\theta}\right) \delta_{\hat{X}_{n, i}^{\theta}}(B)
$$

for $B \in \mathcal{B}(\mathcal{X}) . \tilde{\xi}_{n}^{\theta}(d x)$ and $\tilde{\zeta}_{n}^{\theta}(d x)$ are the (random) measures defined by

$$
\tilde{\xi}_{n}^{\theta}(B)=\xi_{n}^{\theta}(B)-F_{\theta, \boldsymbol{Y}}^{0: n}\left(B \mid \xi_{0}^{\theta}\right), \quad \tilde{\zeta}_{n}^{\theta}(B)=\zeta_{n}^{\theta}(B)-G_{\theta, \boldsymbol{Y}}^{0: n}\left(B \mid \xi_{0}^{\theta}, \zeta_{0}^{\theta}\right)
$$

$\left(\boldsymbol{Y}, F_{\theta, \boldsymbol{y}}^{0: n}(d x \mid \xi), G_{\theta, \boldsymbol{y}}^{0: n}(d x \mid \xi, \zeta)\right.$ are defined in the statement of Lemma 4.1 and (23), (24)). Throughout the proof, we assume (without loss of generality) that $\hat{X}_{0}^{\theta}=\hat{x}_{0}, W_{0}^{\theta}=\mathbf{0}$ for each $\theta \in \Theta$, where $\hat{x}_{0} \in \mathcal{X}^{N}$ is a deterministic vector and $\mathbf{0}$ is the $d \times N$ zero matrix. Consequently, $\xi_{0}^{\theta}(d x), \zeta_{0}^{\theta}(d x)$ are deterministic, independent of $\theta$ and satisfy $\left\|\zeta_{0}^{\theta}\right\|=0$.

In the rest of the proof, let $\theta, y$ be any elements of $Q, \mathcal{Y}$ (respectively), while $\xi, \zeta$ are any elements of $\mathcal{P}(\mathcal{X}), \mathcal{M}_{s}^{d}(\mathcal{X})$. Moreover, let $n$ be any non-negative integer, while $\varphi: \mathcal{X} \rightarrow[-1,1]$ is any function. Then, relying on [33, Theorem 2.1, Proposition 5.1], we conclude that there exists a real number $\tilde{C}_{1, Q} \in[1, \infty)$ (independent of $N$ and depending only on $\left.p_{\theta}\left(x^{\prime} \mid x\right), q_{\theta}(y \mid x)\right)$ such that $\left\|\zeta_{n}^{\theta}\right\| \leq \tilde{C}_{1, Q}$ and

$$
\left|E\left(\int \varphi(x) \tilde{\xi}_{n}^{\theta}(d x) \mid \boldsymbol{Y}\right)\right| \leq \frac{\tilde{C}_{1, Q}}{N}, \quad\left\|E\left(\int \varphi(x) \tilde{\zeta}_{n}^{\theta}(d x) \mid \boldsymbol{Y}\right)\right\| \leq \frac{\tilde{C}_{1, Q}}{N}
$$

almost surely. Similarly, using [10, Theorem 3.1], [20, Theorem 5.8] (or [33, Proposition 6.4]), we deduce that there exists a real number $\tilde{C}_{2, Q} \in[1, \infty)$ (independent of $N$ and depending only on $p_{\theta}\left(x^{\prime} \mid x\right), q_{\theta}(y \mid x)$ ) such that

$$
E\left(\left|\int \varphi(x) \tilde{\xi}_{n}^{\theta}(d x)\right|^{2} \mid \boldsymbol{Y}\right) \leq \frac{\tilde{C}_{2, Q}}{N}, \quad E\left(\left\|\int \varphi(x) \tilde{\zeta}_{n}^{\theta}(d x)\right\|^{2} \mid \boldsymbol{Y}\right) \leq \frac{\tilde{C}_{2, Q}}{N}
$$


almost surely.

It is straightforward to verify

$$
H_{\theta, y}(\xi, \zeta)=H_{\theta, y}^{\prime}(\xi, \zeta)+H_{\theta, y}^{\prime \prime}(\xi, \zeta)
$$

$\left(H_{\theta, y}(\xi, \zeta)\right.$ is defined in $\left.(22)\right)$. It is also easy to show

$$
\frac{1}{A_{1, n}^{\theta}}=\frac{1}{A_{3, n}^{\theta}}-\frac{A_{2, n}^{\theta}}{\left|A_{3, n}^{\theta}\right|^{2}}+\frac{1}{A_{1, n}^{\theta}}\left|\frac{A_{2, n}^{\theta}}{A_{3, n}^{\theta}}\right|^{2} .
$$

Relying on (97), we conclude

$$
\begin{aligned}
H\left(\theta, Z_{n}^{\theta}\right) & =\frac{\sum_{i=1}^{N}\left(q_{\theta}\left(Y_{n} \mid \hat{X}_{n, i}^{\theta}\right)\left(W_{n, i}^{\theta}-N^{-1} \sum_{j=1}^{N} W_{n, j}^{\theta}\right)+\nabla_{\theta} q_{\theta}\left(Y_{n} \mid \hat{X}_{n, i}^{\theta}\right)\right)}{\sum_{i=1}^{N} q_{\theta}\left(Y_{n} \mid \hat{X}_{n, i}^{\theta}\right)} \\
& =\frac{\int q_{\theta}\left(Y_{n} \mid x\right) \zeta_{n}^{\theta}(d x)+\int \nabla_{\theta} q_{\theta}\left(Y_{n} \mid x\right) \xi_{n}^{\theta}(d x)}{\int q_{\theta}\left(Y_{n} \mid x\right) \xi_{n}^{\theta}(d x)}=H_{\theta, Y_{n}}\left(\xi_{n}^{\theta}, \zeta_{n}^{\theta}\right) .
\end{aligned}
$$

Using (98), we also deduce

$$
\begin{aligned}
& H_{\theta, Y_{n}}^{\prime}\left(\xi_{n}^{\theta}, \zeta_{n}^{\theta}\right)-H_{\theta, Y_{n}}^{\prime}\left(F_{\theta, \boldsymbol{Y}}^{0: n}\left(\xi_{0}^{\theta}\right), G_{\theta, \boldsymbol{Y}}^{0: n}\left(\xi_{0}^{\theta}, \zeta_{0}^{\theta}\right)\right)=\frac{B_{2, n}^{\theta}}{A_{3, n}^{\theta}}-\frac{A_{2, n}^{\theta} B_{2, n}^{\theta}}{\left|A_{3, n}^{\theta}\right|^{2}}-\frac{A_{2, n}^{\theta} B_{3, n}^{\theta}}{\left|A_{3, n}^{\theta}\right|^{2}}+\frac{B_{1, n}^{\theta}}{A_{1, n}^{\theta}}\left|\frac{A_{2, n}^{\theta}}{A_{3, n}^{\theta}}\right|^{2}, \\
& H_{\theta, Y_{n}}^{\prime \prime}\left(\xi_{n}^{\theta}, \zeta_{n}^{\theta}\right)-H_{\theta, Y_{n}}^{\prime \prime}\left(F_{\theta, \boldsymbol{Y}}^{0: n}\left(\xi_{0}^{\theta}\right), G_{\theta, \boldsymbol{Y}}^{0: n}\left(\xi_{0}^{\theta}, \zeta_{0}^{\theta}\right)\right)=\frac{C_{2, n}^{\theta}}{A_{3, n}^{\theta}}-\frac{A_{2, n}^{\theta} C_{2, n}^{\theta}}{\left|A_{3, n}^{\theta}\right|^{2}}-\frac{A_{2, n}^{\theta} C_{3, n}^{\theta}}{\left|A_{3, n}^{\theta}\right|^{2}}+\frac{C_{1, n}^{\theta}}{A_{1, n}^{\theta}}\left|\frac{A_{2, n}^{\theta}}{A_{3, n}^{\theta}}\right|^{2} .
\end{aligned}
$$

Moreover, due to Assumption 2.3, we have

$$
A_{1, n}^{\theta}=\int q_{\theta}\left(Y_{n} \mid x\right) \xi_{n}^{\theta}(d x) \geq \varepsilon_{Q}, \quad A_{3, n}^{\theta}=\int q_{\theta}\left(Y_{n} \mid x\right) F_{\theta, \boldsymbol{Y}}^{0: n}\left(d x \mid \xi_{0}^{\theta}\right) \geq \varepsilon_{Q} .
$$

Similarly, owing to Assumption 2.4, we have

$$
\left\|B_{1, n}^{\theta}\right\| \leq \int\left\|\nabla_{\theta} q_{\theta}\left(Y_{n} \mid x\right)\right\| \xi_{n}^{\theta}(d x) \leq K_{1, Q}, \quad\left\|B_{3, n}^{\theta}\right\| \leq \int\left\|\nabla_{\theta} q_{\theta}\left(Y_{n} \mid x\right)\right\| F_{\theta, \boldsymbol{Y}}^{0: n}\left(d x \mid \xi_{0}^{\theta}\right) \leq K_{1, Q} .
$$

Since $\left\|\zeta_{n}^{\theta}\right\| \leq \tilde{C}_{1, Q}$, Assumption 2.3 and Lemma 4.1 yield

$$
\left\|C_{1, n}^{\theta}\right\| \leq \int q_{\theta}\left(Y_{n} \mid x\right)\left|\zeta_{n}^{\theta}\right|(d x) \leq \frac{\tilde{C}_{1, Q}}{\varepsilon_{Q}}, \quad\left\|C_{3, n}^{\theta}\right\| \leq \int q_{\theta}\left(Y_{n} \mid x\right)\left|G_{\theta, \boldsymbol{Y}}^{0: n}\right|\left(d x \mid \xi_{0}^{\theta}, \zeta_{0}^{\theta}\right) \leq \frac{C_{1, Q}}{\varepsilon_{Q}} .
$$

Let $\tilde{C}_{3, Q}=\varepsilon_{Q}^{-4} \sqrt{d} \tilde{C}_{1, Q} C_{1, Q} K_{1, Q}$. Due to Assumptions 2.3, 2.4, we have

$$
0 \leq \varepsilon_{Q} q_{\theta}\left(Y_{n} \mid x\right) \leq 1, \quad\left\|\frac{\nabla_{\theta} q_{\theta}\left(Y_{n} \mid x\right)}{K_{1, Q}}\right\| \leq 1 .
$$

Then, using (95), we conclude

$$
\begin{aligned}
& \left|E\left(A_{2, n}^{\theta} \mid \boldsymbol{Y}\right)\right|=\left|E\left(\int q_{\theta}\left(Y_{n} \mid x\right) \tilde{\xi}_{n}^{\theta}(d x) \mid \boldsymbol{Y}\right)\right| \leq \frac{\tilde{C}_{1, Q}}{\varepsilon_{Q} N}, \\
& \left\|E\left(B_{2, n}^{\theta} \mid \boldsymbol{Y}\right)\right\|=\left\|E\left(\int \nabla_{\theta} q_{\theta}\left(Y_{n} \mid x\right) \tilde{\xi}_{n}^{\theta}(d x) \mid \boldsymbol{Y}\right)\right\| \leq \frac{\sqrt{d} \tilde{C}_{1, Q} K_{1, Q}}{N}, \\
& \left\|E\left(C_{2, n}^{\theta} \mid \boldsymbol{Y}\right)\right\|=\left\|E\left(\int q_{\theta}\left(Y_{n} \mid x\right) \tilde{\zeta}_{n}^{\theta}(d x) \mid \boldsymbol{Y}\right)\right\| \leq \frac{\tilde{C}_{1, Q}}{\varepsilon_{Q} N}
\end{aligned}
$$


almost surely. As $A_{3, n}^{\theta}$ is measurable with respect to $\boldsymbol{Y}$, (102), (106), (107) imply

$$
\begin{aligned}
& \left\|E\left(\frac{B_{2, n}^{\theta}}{A_{3, n}^{\theta}}\right)\right\| \leq E\left(\frac{\left\|E\left(B_{2, n}^{\theta} \mid \boldsymbol{Y}\right)\right\|}{A_{3, n}^{\theta}}\right) \leq \frac{\sqrt{d} \tilde{C}_{1, Q} K_{1, Q}}{\varepsilon_{Q} N} \leq \frac{\tilde{C}_{3, Q}}{N}, \\
& \left\|E\left(\frac{C_{2, n}^{\theta}}{A_{3, n}^{\theta}}\right)\right\| \leq E\left(\frac{\left\|E\left(C_{2, n}^{\theta} \mid \boldsymbol{Y}\right)\right\|}{A_{3, n}^{\theta}}\right) \leq \frac{\tilde{C}_{1, Q}}{\varepsilon_{Q}^{2} N} \leq \frac{\tilde{C}_{3, Q}}{N} .
\end{aligned}
$$

Since $A_{3, n}^{\theta}, B_{3, n}^{\theta}, C_{3, n}^{\theta}$ are measurable with respect to $\boldsymbol{Y},(102)-(105)$ yield

$$
\begin{aligned}
& \left\|E\left(\frac{A_{2, n}^{\theta} B_{3, n}^{\theta}}{\left|A_{3, n}^{\theta}\right|^{2}}\right)\right\| \leq E\left(\frac{\left|E\left(A_{2, n}^{\theta} \mid \boldsymbol{Y}\right)\right|\left\|B_{3, n}^{\theta}\right\|}{\left|A_{3, n}^{\theta}\right|^{2}}\right) \leq \frac{\tilde{C}_{1, Q} K_{1, Q}}{\varepsilon_{Q}^{3} N} \leq \frac{\tilde{C}_{3, Q}}{N}, \\
& \left\|E\left(\frac{A_{2, n}^{\theta} C_{3, n}^{\theta}}{\left|A_{3, n}^{\theta}\right|^{2}}\right)\right\| \leq E\left(\frac{\left|E\left(A_{2, n}^{\theta} \mid \boldsymbol{Y}\right)\right|\left\|C_{3, n}^{\theta}\right\|}{\left|A_{3, n}^{\theta}\right|^{2}}\right) \leq \frac{\tilde{C}_{1, Q} C_{1, Q}}{\varepsilon_{Q}^{4} N} \leq \frac{\tilde{C}_{3, Q}}{N} .
\end{aligned}
$$

Let $\tilde{C}_{4, Q}=\sqrt{d} \varepsilon_{Q}^{-4} \tilde{C}_{2, Q} K_{1, Q}, \tilde{C}_{5, Q}=\varepsilon_{Q}^{-2} \tilde{C}_{1, Q} \tilde{C}_{4, Q} K_{1, Q}$. Relying on Assumptions 2.3, 2.4 and (96), we deduce

$$
\begin{aligned}
& E\left(\left|A_{2, n}^{\theta}\right|^{2} \mid \boldsymbol{Y}\right)=E\left(\left|\int q_{\theta}\left(Y_{n} \mid x\right) \tilde{\xi}_{n}^{\theta}(d x)\right|^{2} \mid \boldsymbol{Y}\right) \leq \frac{\tilde{C}_{2, Q}}{\varepsilon_{Q}^{2} N}, \\
& E\left(\left\|B_{2, n}^{\theta}\right\|^{2} \mid \boldsymbol{Y}\right)=E\left(\left\|\int \nabla_{\theta} q_{\theta}\left(Y_{n} \mid x\right) \tilde{\xi}_{n}^{\theta}(d x)\right\|^{2} \mid \boldsymbol{Y}\right) \leq \frac{d \tilde{C}_{2, Q} K_{1, Q}^{2}}{N}, \\
& E\left(\left\|C_{2, n}^{\theta}\right\|^{2} \mid \boldsymbol{Y}\right)=E\left(\left\|\int q_{\theta}\left(Y_{n} \mid x\right) \tilde{\zeta}_{n}^{\theta}(d x)\right\|^{2} \mid \boldsymbol{Y}\right) \leq \frac{\tilde{C}_{2, Q}}{\varepsilon_{Q}^{2} N}
\end{aligned}
$$

almost surely. Then, Hölder inequality and (102), (112) - (114) imply

$$
\begin{aligned}
& \left\|E\left(\frac{A_{2, n}^{\theta} B_{2, n}^{\theta}}{\left|A_{3, n}^{\theta}\right|^{2}}\right)\right\| \leq\left(E\left(\frac{E\left(\left|A_{2, n}^{\theta}\right|^{2} \mid \boldsymbol{Y}\right)}{\left|A_{3, n}^{\theta}\right|^{2}}\right)\right)^{1 / 2}\left(E\left(\frac{E\left(\left\|B_{2, n}^{\theta}\right\|^{2} \mid \boldsymbol{Y}\right)}{\left|A_{3, n}^{\theta}\right|^{2}}\right)\right)^{1 / 2} \leq \frac{\sqrt{d} \tilde{C}_{2, Q} K_{1, Q}}{\varepsilon_{Q}^{3} N} \leq \frac{\tilde{C}_{4, Q}}{N}, \\
& \left\|E\left(\frac{A_{2, n}^{\theta} C_{2, n}^{\theta}}{\left|A_{3, n}^{\theta}\right|^{2}}\right)\right\| \leq\left(E\left(\frac{E\left(\left|A_{2, n}^{\theta}\right|^{2} \mid \boldsymbol{Y}\right)}{\left|A_{3, n}^{\theta}\right|^{2}}\right)\right)^{1 / 2}\left(E\left(\frac{E\left(\left\|C_{2, n}^{\theta}\right\|^{2} \mid \boldsymbol{Y}\right)}{\left|A_{3, n}^{\theta}\right|^{2}}\right)\right)^{1 / 2} \leq \frac{\tilde{C}_{2, Q}}{\varepsilon_{Q}^{4} N} \leq \frac{\tilde{C}_{4, Q}}{N}
\end{aligned}
$$

Moreover, due to (102), (112), we have

$$
E\left(\left|\frac{A_{2, n}^{\theta}}{A_{3, n}^{\theta}}\right|^{2}\right)=E\left(\frac{E\left(\left|A_{2, n}^{\theta}\right|^{2} \mid \boldsymbol{Y}\right)}{\left|A_{3, n}^{\theta}\right|^{2}}\right) \leq \frac{\tilde{C}_{2, Q}}{\varepsilon_{Q}^{4} N} \leq \frac{\tilde{C}_{4, Q}}{N} .
$$

Owing to (102), (103), (104), (117), we also have

$$
\begin{aligned}
& \left\|E\left(\frac{B_{1, n}^{\theta}}{A_{1, n}^{\theta}}\left|\frac{A_{2, n}^{\theta}}{A_{3, n}^{\theta}}\right|^{2}\right)\right\| \leq E\left(\frac{\left\|B_{1, n}^{\theta}\right\|}{A_{1, n}^{\theta}}\left|\frac{A_{2, n}^{\theta}}{A_{3, n}^{\theta}}\right|^{2}\right) \leq \frac{K_{1, Q}}{\varepsilon_{Q}} E\left(\left|\frac{A_{2, n}^{\theta}}{A_{3, n}^{\theta}}\right|^{2}\right) \leq \frac{\tilde{C}_{4, Q} K_{1, Q}}{\varepsilon_{Q} N} \leq \frac{\tilde{C}_{5, Q}}{N}, \\
& \left\|E\left(\frac{C_{1, n}^{\theta}}{A_{1, n}^{\theta}}\left|\frac{A_{2, n}^{\theta}}{A_{3, n}^{\theta}}\right|^{2}\right)\right\| \leq E\left(\frac{\left\|C_{1, n}^{\theta}\right\|}{A_{1, n}^{\theta}}\left|\frac{A_{2, n}^{\theta}}{A_{3, n}^{\theta}}\right|^{2}\right) \leq \frac{\tilde{C}_{1, Q}}{\varepsilon_{Q}^{2}} E\left(\left|\frac{A_{2, n}^{\theta}}{A_{3, n}^{\theta}}\right|^{2}\right) \leq \frac{\tilde{C}_{1, Q} \tilde{C}_{4, Q}}{\varepsilon_{Q}^{2} N} \leq \frac{\tilde{C}_{5, Q}}{N} .
\end{aligned}
$$

Let $M_{Q}=4\left(\tilde{C}_{3, Q}+\tilde{C}_{4, Q}+\tilde{C}_{5, Q}\right)$. Then, (100), (108), (110), (115), (118) imply

$$
\left\|E\left(H_{\theta, Y_{n}}^{\prime}\left(\xi_{n}^{\theta}, \zeta_{n}^{\theta}\right)-H_{\theta, Y_{n}}^{\prime}\left(F_{\theta, \boldsymbol{Y}}^{0: n}\left(\xi_{0}^{\theta}\right), G_{\theta, \boldsymbol{Y}}^{0: n}\left(\xi_{0}^{\theta}, \zeta_{0}^{\theta}\right)\right)\right)\right\| \leq \frac{2 \tilde{C}_{3, Q}+\tilde{C}_{4, Q}+\tilde{C}_{5, Q}}{N} \leq \frac{M_{Q}}{2 N} .
$$


Similarly, (101), (109), (111), (116), (119) yield

$$
\left\|E\left(H_{\theta, Y_{n}}^{\prime \prime}\left(\xi_{n}^{\theta}, \zeta_{n}^{\theta}\right)-H_{\theta, Y_{n}}^{\prime \prime}\left(F_{\theta, \boldsymbol{Y}}^{0: n}\left(\xi_{0}^{\theta}\right), G_{\theta, \boldsymbol{Y}}^{0: n}\left(\xi_{0}^{\theta}, \zeta_{0}^{\theta}\right)\right)\right)\right\| \leq \frac{2 \tilde{C}_{3, Q}+\tilde{C}_{4, Q}+\tilde{C}_{5, Q}}{N} \leq \frac{M_{Q}}{2 N} .
$$

Combining this with (97), (99), we get

$$
\begin{aligned}
\left\|E\left(H\left(\theta, Z_{n}^{\theta}\right)-H_{\theta, Y_{n}}\left(F_{\theta, \boldsymbol{Y}}^{0: n}\left(\xi_{0}^{\theta}\right), G_{\theta, \boldsymbol{Y}}^{0: n}\left(\xi_{0}^{\theta}, \zeta_{0}^{\theta}\right)\right)\right)\right\| & \left\|E\left(H_{\theta, Y_{n}}^{\prime}\left(\xi_{n}^{\theta}, \zeta_{n}^{\theta}\right)-H_{\theta, Y_{n}}^{\prime}\left(F_{\theta, \boldsymbol{Y}}^{0: n}\left(\xi_{0}^{\theta}\right), G_{\theta, \boldsymbol{Y}}^{0: n}\left(\xi_{0}^{\theta}, \zeta_{0}^{\theta}\right)\right)\right)\right\| \\
& +\left\|E\left(H_{\theta, Y_{n}}^{\prime \prime}\left(\xi_{n}^{\theta}, \zeta_{n}^{\theta}\right)-H_{\theta, Y_{n}}^{\prime \prime}\left(F_{\theta, \boldsymbol{Y}}^{0: n}\left(\xi_{0}^{\theta}\right), G_{\theta, \boldsymbol{Y}}^{0: n}\left(\xi_{0}^{\theta}, \zeta_{0}^{\theta}\right)\right)\right)\right\| \\
\leq & \frac{M_{Q}}{N} .
\end{aligned}
$$

Hence, we have

$$
\begin{aligned}
\|h(\theta)-\nabla l(\theta)\| \leq & \left\|E\left(H\left(\theta, Z_{n}^{\theta}\right)\right)-h(\theta)\right\|+\left\|E\left(H_{\theta, Y_{n}}\left(F_{\theta, \boldsymbol{Y}}^{0: n}\left(\xi_{0}^{\theta}\right), G_{\theta, \boldsymbol{Y}}^{0: n}\left(\xi_{0}^{\theta}, \zeta_{0}^{\theta}\right)\right)\right)-\nabla l(\theta)\right\| \\
& +\left\|E\left(H\left(\theta, Z_{n}^{\theta}\right)-H_{\theta, Y_{n}}\left(F_{\theta, \boldsymbol{Y}}^{0: n}\left(\xi_{0}^{\theta}\right), G_{\theta, \boldsymbol{Y}}^{0: n}\left(\xi_{0}^{\theta}, \zeta_{0}^{\theta}\right)\right)\right)\right\| \\
\leq & \left\|E\left(H\left(\theta, Z_{n}^{\theta}\right)\right)-h(\theta)\right\|+\left\|E\left(H_{\theta, Y_{n}}\left(F_{\theta, \boldsymbol{Y}}^{0: n}\left(\xi_{0}^{\theta}\right), G_{\theta, \boldsymbol{Y}}^{0: n}\left(\xi_{0}^{\theta}, \zeta_{0}^{\theta}\right)\right)\right)-\nabla l(\theta)\right\|+\frac{M_{Q}}{N} .
\end{aligned}
$$

Then, letting $n \rightarrow \infty$ and using Lemmas 4.1, 5.4, we conclude that (93) holds.

\section{Proof of Main Results}

In this section, we study the Monte Carlo estimation of the log-likelihood rate gradient $\nabla l(\theta)$ (see Lemma 6.1). We also study the analytical properties of $l(\theta)$ (see Lemma 6.2). Using these results (together with the results of [30] - [31]), we prove Theorem 2.1.

Throughout the section, the following notation is used. $\left\{\zeta_{n}\right\}_{n \geq 0},\left\{\eta_{n}\right\}_{n \geq 0}$ and $\left\{\xi_{n}\right\}_{n \geq 0}$ are the stochastic processes defined by

$$
\zeta_{n}=H\left(\theta_{n}, Z_{n+1}\right)-h\left(\theta_{n}\right), \quad \eta_{n}=h\left(\theta_{n}\right)-\nabla l\left(\theta_{n}\right), \quad \xi_{n}=\zeta_{n}+\eta_{n}
$$

for $n \geq 0\left(H(\theta, z), h(\theta),\left\{Z_{n}\right\}_{n \geq 0}\right.$ are specified in (29), (16), (42)). Then, using (14), it is easy to show that (19) holds for each $n \geq 0$.

Remark. Due to (19), algorithm (1) - (4) is stochastic gradient search which maximizes log-likelihood rate $l(\theta)$, while $\left\{\xi_{n}\right\}_{n \geq 0}$ can be interpreted as noise in the (Monte Carlo) estimation of $\nabla l(\theta)$. We also recall here that $Q$ is any compact set satisfying $Q \subset \Theta$.

Lemma 6.1. Let Assumptions 2.1 - 2.4 hold. Then, relations

$$
\lim _{n \rightarrow \infty} \sup _{k \geq n}\left\|\sum_{i=n}^{k} \alpha_{i} \zeta_{i}\right\|=0, \quad \limsup _{n \rightarrow \infty}\left\|\eta_{n}\right\| \leq \frac{M_{Q}}{N}
$$

hold almost surely on $\Lambda_{Q}\left(\Lambda_{Q}\right.$ is defined in (6)).

Proof. Let $\tau_{Q}$ be the stopping time defined in Lemma 5.5. Moreover, let $\tilde{\Lambda}_{Q}$ be the event defined by $\tilde{\Lambda}_{Q}=\bigcap_{n=0}^{\infty}\left\{\theta_{n} \in Q\right\}$. Hence, on $\Lambda_{Q}, \theta_{n} \in Q$ for all, but finitely many $n \geq 0$. Then, using Lemma 5.6, we conclude that the second part of (120) holds almost surely on $\Lambda_{Q}$.

Throughout the rest of the proof, the following notation is used. $\mathcal{F}_{k}$ is the $\sigma$-algebra defined by $\mathcal{F}_{k}=$ $\sigma\left\{\theta_{0}, Z_{0}, \cdots, \theta_{k}, Z_{k}\right\}$ for $k \geq 0 . n$ is any positive integer, while $\zeta_{1, n}, \zeta_{2, n}$ and $\zeta_{3, n}$ are the random variables defined by

$\zeta_{1, n}=\tilde{H}\left(\theta_{n}, Z_{n+1}\right)-(\Pi \tilde{H})\left(\theta_{n}, Z_{n}\right), \quad \zeta_{2, n}=(\Pi \tilde{H})\left(\theta_{n}, Z_{n}\right)-(\Pi \tilde{H})\left(\theta_{n-1}, Z_{n}\right), \quad \zeta_{3, n}=-(\Pi \tilde{H})\left(\theta_{n}, Z_{n+1}\right)$. 
Then, for $l \geq k>1$, it is straightforward to verify

$$
\sum_{i=k}^{l} \alpha_{i} \zeta_{i}=\sum_{i=k}^{l} \alpha_{i} \zeta_{1, i}+\sum_{i=k}^{l} \alpha_{i} \zeta_{2, i}+\sum_{i=k}^{l}\left(\alpha_{i}-\alpha_{i+1}\right) \zeta_{3, i}+\alpha_{l+1} \zeta_{3, l}-\alpha_{k} \zeta_{3, k-1}
$$

As a direct consequence of Lemmas 5.4, 5.5, we have

$$
\left\|\zeta_{1, n}\right\| I_{\left\{\tau_{Q}>n\right\}} \leq C_{5, Q}\left(2+\left\|W_{n} \Lambda\right\|+\left\|W_{n+1} \Lambda\right\|\right) I_{\left\{\tau_{Q}>n\right\}} \leq 4 C_{5, Q} C_{6, Q}\left(1+\left\|W_{0} \Lambda\right\|\right) .
$$

Since $W_{0}$ is measurable with respect to $\mathcal{F}_{0}$, Assumption 2.1 yields

$$
E\left(\sum_{n=1}^{\infty} \alpha_{n}^{2}\left\|\zeta_{1, n}\right\|^{2} I_{\left\{\tau_{Q}>n\right\}} \mid \mathcal{F}_{0}\right) \leq 16 C_{5, Q}^{2} C_{6, Q}^{2}\left(1+\left\|W_{0} \Lambda\right\|\right)^{2}\left(\sum_{n=0}^{\infty} \alpha_{n}^{2}\right)<\infty
$$

almost surely. As $\left\{\tau_{Q}>n\right\} \in \mathcal{F}_{n}$, we also have

$$
E\left(\zeta_{1, n} I_{\left\{\tau_{Q}>n\right\}} \mid \mathcal{F}_{n}\right)=\left(E\left(\tilde{H}\left(\theta_{n}, Z_{n+1}\right) \mid \mathcal{F}_{n}\right)-(\Pi \tilde{H})\left(\theta_{n}, Z_{n}\right)\right) I_{\left\{\tau_{Q}>n\right\}}=0
$$

almost surely. Then, Doob theorem and (122) imply that $\sum_{n=1}^{\infty} \alpha_{n} \zeta_{1, n} I_{\left\{\tau_{Q}>n\right\}}$ is almost surely convergent. Since $\tilde{\Lambda}_{Q} \subseteq\left\{\tau_{Q}>n\right\}, \sum_{n=1}^{\infty} \alpha_{n} \zeta_{1, n}$ converges almost surely on $\tilde{\Lambda}_{Q}$.

Due to Lemmas 5.4, 5.5 and (14), we have

$$
\begin{aligned}
\left\|\zeta_{2, n}\right\| I_{\left\{\tau_{Q}>n\right\}} \leq C_{5, Q}\left\|\theta_{n}-\theta_{n-1}\right\|\left(1+\left\|W_{n} \Lambda\right\|\right) I_{\left\{\tau_{Q}>n\right\}} & =C_{5, Q} \alpha_{n-1}\left\|H\left(\theta_{n-1}, Z_{n}\right)\right\|\left(1+\left\|W_{n} \Lambda\right\|\right) I_{\left\{\tau_{Q}>n\right\}} \\
& \leq C_{5, Q}^{2} \alpha_{n-1}\left(1+\left\|W_{n} \Lambda\right\|\right)^{2} I_{\left\{\tau_{Q}>n\right\}} \\
& \leq 4 C_{5, Q}^{2} C_{6, Q}^{2} \alpha_{n-1}\left(1+\left\|W_{0} \Lambda\right\|\right)^{2} .
\end{aligned}
$$

Combining this with Assumption 2.1, we get

$$
\begin{aligned}
\sum_{n=1}^{\infty} \alpha_{n}\left\|\zeta_{2, n}\right\| I_{\left\{\tau_{Q}>n\right\}} & \leq 4 C_{5, Q}^{2} C_{6, Q}^{2}\left(1+\left\|W_{0} \Lambda\right\|\right)^{2}\left(\sum_{n=0}^{\infty} \alpha_{n} \alpha_{n+1}\right) \\
& \leq 2 C_{5, Q}^{2} C_{6, Q}^{2}\left(1+\left\|W_{0} \Lambda\right\|\right)^{2}\left(\sum_{n=0}^{\infty} \alpha_{n}^{2}\right)<\infty
\end{aligned}
$$

Hence, $\sum_{n=0}^{\infty} \alpha_{n} \zeta_{2, n} I_{\left\{\tau_{Q}>n\right\}}$ converges almost surely. Therefore, $\sum_{n=0}^{\infty} \alpha_{n} \zeta_{2, n}$ is convergent almost surely on $\tilde{\Lambda}_{Q}$.

As a direct consequence of Lemmas 5.4, 5.5, we have

$$
\left\|\zeta_{3, n}\right\| I_{\left\{\tau_{Q}>n\right\}} \leq C_{5, Q}\left(1+\left\|W_{n+1} \Lambda\right\|\right) I_{\left\{\tau_{Q}>n\right\}} \leq 2 C_{5, Q} C_{6, Q}\left(1+\left\|W_{0} \Lambda\right\|\right) .
$$

Consequently, Assumption 2.1 yields

$$
\begin{aligned}
& \sum_{n=1}^{\infty} \alpha_{n+1}^{2}\left\|\zeta_{3, n}\right\|^{2} I_{\left\{\tau_{Q}>n\right\}} \leq 4 C_{5, Q}^{2} C_{6, Q}^{2}\left(1+\left\|W_{0} \Lambda\right\|\right)^{2}\left(\sum_{n=0}^{\infty} \alpha_{n}^{2}\right)<\infty, \\
& \sum_{n=1}^{\infty}\left|\alpha_{n}-\alpha_{n+1}\right|\left\|\zeta_{3, n}\right\| I_{\left\{\tau_{Q}>n\right\}} \leq 2 C_{5, Q} C_{6, Q}\left(1+\left\|W_{0} \Lambda\right\|\right)\left(\sum_{n=0}^{\infty}\left|\alpha_{n}-\alpha_{n+1}\right|\right)<\infty .
\end{aligned}
$$

Therefore, we have

$$
\lim _{n \rightarrow \infty} \alpha_{n+1} \zeta_{3, n} I_{\left\{\tau_{Q}>n\right\}}=0
$$

almost surely. Hence, $\lim _{n \rightarrow \infty} \alpha_{n+1} \zeta_{3, n}=0$ almost surely on $\tilde{\Lambda}_{Q}$. Moreover, due to (124),

$$
\sum_{n=1}^{\infty}\left(\alpha_{n}-\alpha_{n+1}\right) \zeta_{3, n} I_{\left\{\tau_{Q}>n\right\}}
$$


is almost surely convergent. Thus, $\sum_{n=1}^{\infty}\left(\alpha_{n}-\alpha_{n+1}\right) \zeta_{3, n}$ converges almost surely on $\tilde{\Lambda}_{Q}$. Since $\sum_{n=1}^{\infty} \alpha_{n} \zeta_{1, n}, \sum_{n=1}^{\infty} \alpha_{n} \zeta_{2, n}$ are almost surely convergent on $\tilde{\Lambda}_{Q}$, (121) implies that $\sum_{n=0}^{\infty} \alpha_{n} \zeta_{n}$ converges almost surely on $\tilde{\Lambda}_{Q}$, too. As $Q$ is any compact set in $\Theta$, we conclude that $\sum_{n=0}^{\infty} \alpha_{n} \zeta_{n}$ is almost surely convergent on $\left\{\sup _{n \geq 0}\left\|\theta_{n}\right\|<\infty, \inf _{n \geq 0} d\left(\theta_{n}, \Theta^{c}\right)>0\right\}$. Consequently, the first part of (122) holds almost surely on $\Lambda_{Q}$.

Lemma 6.2. Let Assumptions 2.2-2.4 hold. Then, the following is true:

(i) $l(\theta)$ is well-defined for each $\theta \in \Theta$. Moreover, $l(\theta)$ is Lipschitz continuously differentiable on $\Theta$.

(ii) If Assumption 2.5 also holds, then $l(\theta)$ is p-times differentiable on $\Theta$.

(iii) If Assumption 2.6 also holds, then $l(\theta)$ is real-analytic on $\Theta$.

Proof. (i) See Lemma 4.1. (ii) See [32, Theorem 3.1]. (iii) See [31, Theorem 2.1].

Proof of Theorem 2.1. Let $\eta=\lim \sup _{n \rightarrow \infty}\left\|\eta_{n}\right\|$. Then, Lemma 6.1 yields $\eta \leq M_{Q} / N$ almost surely on $\Lambda_{Q}$. Moreover, due to Assumption 2.1 and Lemmas 6.1, 6.2, Algorithm (19) satisfies all conditions which [30, Theorem 2.1] is based on. Consequently, [30, Theorem 2.1] implies that there exist a function $\psi_{Q}(t)$ and real numbers $r_{Q}, L_{1, Q}, L_{2, Q}$ with the properties specified in the statement Theorem 2.1.

\section{Appendix 1}

In this section, we present results on stochastic matrices which are needed for the proof of Lemmas 5.3 and 5.5. Here, we rely on the following notation. $\|\cdot\|$ denotes the Euclidean vector norm and Frobenius matrix norm, while $\|\cdot\|_{1}$ stands for the $l_{1}$ vector norm. $N \geq 1$ is an integer. $\mathcal{P}^{N}$ is the set of $N$ dimensional (column) probability vectors, while $\mathcal{P}^{N \times N}$ is the set of $N \times N$ (column) stochastic matrices (i.e., $A \in \mathcal{P}^{N \times N}$ if and only if the columns of $A$ are elements of $\mathcal{P}^{N}$ ). $e$ is the $N$-dimensional vector whose all elements are one. For $1 \leq i \leq N, e_{i}$ is the $i$-th standard unit vector in $\mathbb{R}^{N}$ (i.e., $e_{i}$ is the element of $\mathcal{P}^{N}$ whose $i$-th element is one). $I$ is the $N \times N$ unit matrix. $\Lambda$ is the matrix defined by $\Lambda=I-e e^{T} / N$. For $A \in \mathcal{P}^{N \times N}, \tau(A)$ is the (Dobrushin) ergodicity coefficient, i.e.,

$$
\tau(A)=\frac{1}{2} \max _{1 \leq j, j^{\prime} \leq N} \sum_{i=1}^{N}\left|A_{i, j}-A_{i, j^{\prime}}\right|,
$$

where $A_{i, j}$ is the $(i, j)$ entry of $A$.

Lemma A1.1. (i) If $A \in \mathcal{P}^{N \times N}$, then we have

$$
\tau(A)=1-\min _{1 \leq j, j^{\prime} \leq N} \sum_{i=1}^{N} \min \left\{A_{i, j}, A_{i, j^{\prime}}\right\},
$$

where $A_{i, j}$ is the $(i, j)$ entry of $A$.

(ii) If $A \in \mathcal{P}^{N \times N}$ and $z, z^{\prime} \in \mathcal{P}^{N}$, then we have

$$
\left\|A\left(z-z^{\prime}\right)\right\|_{1} \leq \tau(A)\left\|z-z^{\prime}\right\|_{1}
$$

Moreover, if $A, A^{\prime} \in \mathcal{P}^{N \times N}$, then $\tau\left(A A^{\prime}\right) \leq \tau(A) \tau\left(A^{\prime}\right)$.

Proof. (i) See [7, Definition 15.2.1, Equation (15.9)]. (ii) See [7, Theorems 15.2.4, 15.2.5].

Lemma A1.2. Let $\left\{A_{n}\right\}_{n \geq 1},\left\{B_{n}\right\}_{n \geq 1}$ and $\left\{C_{n}\right\}_{n \geq 1}$ be sequences in $\mathcal{P}^{N \times N}$. Moreover, let $a, b, c \in \mathbb{R}^{N}$. Assume the following:

(i) There exists a real number $\alpha \in(0,1)$ such that $\min \left\{A_{n, i, j}, B_{n, i, j}, C_{n, i, j}\right\} \geq \alpha / N$ for each $1 \leq i, j \leq N$, $n \geq 1$, where $A_{n, i, j}, B_{n, i, j}, C_{n, i, j}$ are the $(i, j)$ entries of $A_{n}, B_{n}, C_{n}$ (respectively). 
(ii) $e^{T} a=e^{T} b=e^{T} c=0$.

Then, we have

$$
\begin{aligned}
& \left\|A_{1} \cdots A_{n} \Lambda\right\| \leq K \beta^{n} \\
& \left\|A_{1} \cdots A_{n} a\right\| \leq K \beta^{n}\|a\|, \\
& \left\|B_{1} \cdots B_{n} b-C_{1} \cdots C_{n} c\right\| \leq K \beta^{n}(\|b\|+\|c\|) \sum_{i=1}^{n}\left\|B_{i}-C_{i}\right\|+K \beta^{n}\|b-c\|
\end{aligned}
$$

for each $n \geq 1$, where $\beta=1-\alpha$ and $K=4 \beta^{-1} N$.

Proof. Throughout the proof, the following notation is used. $n, k, l$ are any integers satisfying $n \geq 1$, $l \geq k \geq 0 . \tilde{A}_{k, k}, \tilde{B}_{k, k}, \tilde{C}_{k, k}$ and $\tilde{A}_{k, m}, \tilde{B}_{k, m}, \tilde{C}_{k, m}$ are the matrices defined by $\tilde{A}_{k, k}=\tilde{B}_{k, k}=\tilde{C}_{k, k}=I$ and

$$
\tilde{A}_{k, m}=A_{k+1} \cdots A_{m}, \quad \tilde{B}_{k, m}=B_{k+1} \cdots B_{m}, \quad \tilde{C}_{k, m}=C_{k+m} \cdots C_{m}
$$

for $m>k \geq 0$. Then, using Lemma A1.1, we conclude

$$
\tau\left(\tilde{A}_{k, m}\right) \leq \tau\left(A_{k+1}\right) \cdots \tau\left(A_{m}\right) \leq \beta^{m-k} .
$$

Relying on the same lemma, we deduce

$$
\tau\left(A_{n}\right)=1-\min _{1 \leq j, j^{\prime} \leq N} \sum_{i=1}^{N} \min \left\{A_{n, i, j}, A_{n, i, j^{\prime}}\right\} \leq 1-\alpha=\beta .
$$

Noticing $e_{i}, e / N \in \mathcal{P}^{N}$ and applying Lemma A1.1 again, we get

$$
\left\|\tilde{A}_{k, l}\left(e_{i}-\frac{e}{N}\right)\right\|_{1} \leq \tau\left(\tilde{A}_{k, l}\right)\left\|e_{i}-\frac{e}{N}\right\|_{1} \leq 2 \beta^{l-k}
$$

for $1 \leq i \leq N$. Since $\tilde{A}_{k, l}\left(e_{i}-\frac{e}{N}\right)$ is the $i$-th column of $\tilde{A}_{k, l} \Lambda$, (125) yields

$$
\left\|\tilde{A}_{k, l} \Lambda\right\| \leq N^{1 / 2} \max _{1 \leq i \leq N}\left\|\tilde{A}_{k, l}\left(e_{i}-\frac{e}{N}\right)\right\|_{1} \leq 2 N^{1 / 2} \beta^{l-k} .
$$

Hence, we get

$$
\left\|A_{1} \cdots A_{n} \Lambda\right\|=\left\|\tilde{A}_{0, n} \Lambda\right\| \leq 2 N^{1 / 2} \beta^{n} \leq K \beta^{n} .
$$

Moreover, we have

$$
\tilde{A}_{k, l} \Lambda a=\tilde{A}_{k, l} a-\frac{\tilde{A}_{k, l} e}{N} e^{T} a=A_{k, l} a .
$$

Consequently, (126) implies

$$
\left\|\tilde{A}_{k, l} a\right\|=\left\|\tilde{A}_{k, l} \Lambda a\right\| \leq\left\|\tilde{A}_{k, l} \Lambda\right\|\|a\| \leq 2 N^{1 / 2} \beta^{l-k}\|a\| .
$$

Thus, we get

$$
\left\|A_{1} \cdots A_{n} a\right\|=\left\|\tilde{A}_{0, n} a\right\| \leq 2 N^{1 / 2} \beta^{n}\|a\| \leq K \beta^{n}\|a\| .
$$

Since $e^{T} B_{n}=e^{T} C_{n}=e^{T}$, we have

$$
\Lambda\left(B_{n}-C_{n}\right)=B_{n}-C_{n}-\frac{e}{N}\left(e^{T} B_{n}-e^{T} C_{n}\right)=B_{n}-C_{n} .
$$

Therefore, we get

$$
\begin{aligned}
\tilde{B}_{0, n} b-\tilde{C}_{0, n} c & =\sum_{i=1}^{n} \tilde{B}_{0, i-1}\left(B_{i}-C_{i}\right) \tilde{C}_{i, n} b+\tilde{C}_{0, n}(b-c) \\
& =\sum_{i=1}^{n} \tilde{B}_{0, i-1} \Lambda\left(B_{i}-C_{i}\right) \tilde{C}_{i, n} b+\tilde{C}_{0, n}(b-c) .
\end{aligned}
$$


Then, applying (126), (127) to $\left\{B_{n}\right\}_{n \geq 1},\left\{C_{n}\right\}_{n \geq 1}, b, c$, we get

$$
\begin{aligned}
\left\|\tilde{B}_{0, n} b-\tilde{C}_{0, n} c\right\| & \leq \sum_{i=1}^{n}\left\|\tilde{B}_{0, i-1} \Lambda\right\|\left\|B_{i}-C_{i}\right\|\left\|\tilde{C}_{i, n} b\right\|+\left\|\tilde{C}_{0, n}(b-c)\right\| \\
& \leq 4 N \beta^{n-1}\|b\| \sum_{i=1}^{n}\left\|B_{i}-C_{i}\right\|+2 N^{1 / 2} \beta^{n}\|b-c\| \\
& \leq K \beta^{n}(\|b\|+\|c\|) \sum_{i=1}^{n}\left\|B_{i}-C_{i}\right\|+K \beta^{n}\|b-c\| .
\end{aligned}
$$

Hence, we have

$$
\left\|B_{1} \cdots B_{n} b-C_{1} \cdots C_{n} c\right\|=\left\|\tilde{B}_{0, n} b-\tilde{C}_{0, n} c\right\| \leq K \beta^{n}(\|b\|+\|c\|) \sum_{i=1}^{n}\left\|B_{i}-C_{i}\right\|+K \beta^{n}\|b-c\| .
$$

\section{Appendix 2}

In this section, we prove Lemma 4.1. We rely on the following notation. $\tilde{r}_{\theta}\left(y, x^{\prime} \mid x\right)$ is the function defined for $\theta \in \Theta, x, x^{\prime} \in \mathcal{X}, y \in \mathcal{Y}$ by

$$
\tilde{r}_{\theta}\left(y, x^{\prime} \mid x\right)=q_{\theta}\left(y \mid x^{\prime}\right) p_{\theta}\left(x^{\prime} \mid x\right) .
$$

$\tilde{h}_{\theta, y}(x \mid \xi, \zeta)$ and $\tilde{H}_{\theta, y}(\xi, \zeta)$ are the functions defined for $\xi \in \mathcal{P}(\mathcal{X}), \zeta \in \mathcal{M}_{s}^{d}(\mathcal{X})$ by

$$
\tilde{h}_{\theta, y}(x \mid \xi, \zeta)=\frac{\int \tilde{r}_{\theta}\left(y, x \mid x^{\prime}\right) \zeta\left(d x^{\prime}\right)+\int \nabla_{\theta} \tilde{r}_{\theta}\left(y, x \mid x^{\prime}\right) \xi\left(d x^{\prime}\right)}{\iint \tilde{r}_{\theta}\left(y, x^{\prime \prime} \mid x^{\prime}\right) \mu\left(d x^{\prime \prime}\right) \xi\left(d x^{\prime}\right)}, \quad \tilde{H}_{\theta, y}(\xi, \zeta)=\int \tilde{h}_{\theta, y}(x \mid \xi, \zeta) \mu(d x),
$$

while $\tilde{f}_{\theta, y}(x \mid \xi)$ and $\tilde{g}_{\theta, y}(x \mid \xi, \zeta)$ are defined as

$$
\tilde{f}_{\theta, y}(x \mid \xi)=\frac{\int \tilde{r}_{\theta}\left(y, x \mid x^{\prime}\right) \xi\left(d x^{\prime}\right)}{\iint \tilde{r}_{\theta}\left(y, x^{\prime \prime} \mid x\right) \mu\left(d x^{\prime \prime}\right) \xi\left(d x^{\prime}\right)}, \quad \tilde{g}_{\theta, y}(x \mid \xi, \zeta)=\tilde{h}_{\theta, y}(x \mid \xi, \zeta)-\tilde{f}_{\theta, y}(x \mid \xi) \tilde{H}_{\theta, y}(\xi, \zeta) .
$$

$\tilde{F}_{\theta, y}(d x \mid \xi)$ and $\tilde{G}_{\theta, y}(d x \mid \xi, \zeta)$ are the measures defined for $B \in \mathcal{B}(\mathcal{X})$ by

$$
\tilde{F}_{\theta, y}(B \mid \xi)=\int_{B} \tilde{f}_{\theta, y}(x \mid \xi) \mu(d x), \quad \tilde{G}_{\theta, y}(B \mid \xi, \zeta)=\int_{B} \tilde{g}_{\theta, y}(x \mid \xi, \zeta) \mu(d x) .
$$

Measures $\tilde{F}_{\theta, y}(d x \mid \xi)$ and $\tilde{G}_{\theta, y}(d x \mid \xi, \zeta)$ are also denoted by $\tilde{F}_{\theta, y}(\xi)$ and $\tilde{G}_{\theta, y}(\xi, \zeta)$ (short-hand notation). $\tilde{r}_{\theta, \boldsymbol{y}}^{m: n}\left(x^{\prime} \mid x\right)$ is the function recursively defined by

$$
\tilde{r}_{\theta, \boldsymbol{y}}^{m: m+1}\left(x^{\prime} \mid x\right)=\tilde{r}_{\theta}\left(y_{m+1}, x^{\prime} \mid x\right), \quad \tilde{r}_{\theta, \boldsymbol{y}}^{m: n+1}\left(x^{\prime} \mid x\right)=\int \tilde{r}_{\theta, \boldsymbol{y}}^{n: n+1}\left(x^{\prime} \mid x^{\prime \prime}\right) \tilde{r}_{\theta, \boldsymbol{y}}^{m: n}\left(x^{\prime \prime} \mid x\right) \mu\left(d x^{\prime \prime}\right)
$$

for $n>m \geq 0$ and a sequence $\boldsymbol{y}=\left\{y_{n}\right\}_{n \geq 0}$ in $\mathcal{Y} . \tilde{h}_{\theta, \boldsymbol{y}}^{m: n}(x \mid \xi, \zeta)$ and $\tilde{H}_{\theta, \boldsymbol{y}}^{m: n}(\xi, \zeta)$ are the functions defined by

$$
h_{\theta, \boldsymbol{y}}^{m: n}(x \mid \xi, \zeta)=\frac{\int r_{\theta, \boldsymbol{y}}^{m: n}\left(x \mid x^{\prime}\right) \zeta\left(d x^{\prime}\right)+\int \nabla_{\theta} r_{\theta, \boldsymbol{y}}^{m: n}\left(x \mid x^{\prime}\right) \xi\left(d x^{\prime}\right)}{\iint r_{\theta, \boldsymbol{y}}^{m: n}\left(x^{\prime \prime} \mid x^{\prime}\right) \xi\left(d x^{\prime}\right) \mu\left(d x^{\prime \prime}\right)}, \quad \tilde{H}_{\theta, \boldsymbol{y}}^{m: n}(\xi, \zeta)=\int \tilde{h}_{\theta, \boldsymbol{y}}^{m: n}(x \mid \xi, \zeta) \mu(d x),
$$

while $\tilde{f}_{\theta, \boldsymbol{y}}^{m: n}(x \mid \xi)$ and $\tilde{g}_{\theta, \boldsymbol{y}}^{m: n}(x \mid \xi, \zeta)$ are defined as

$$
\tilde{f}_{\theta, \boldsymbol{y}}^{m: n}(x \mid \xi)=\frac{\int \tilde{r}_{\theta, \boldsymbol{y}}^{m: n}\left(x \mid x^{\prime}\right) \xi\left(d x^{\prime}\right)}{\iint \tilde{r}_{\theta, \boldsymbol{y}}^{m: n}\left(x^{\prime \prime} \mid x^{\prime}\right) \xi\left(d x^{\prime}\right) \mu\left(d x^{\prime \prime}\right)}, \quad \tilde{g}_{\theta, \boldsymbol{y}}^{m: n}(x \mid \xi, \zeta)=\tilde{h}_{\theta, \boldsymbol{y}}^{m: n}(x \mid \xi, \zeta)-\tilde{f}_{\theta, \boldsymbol{y}}^{m: n}(x \mid \xi) \tilde{H}_{\theta, \boldsymbol{y}}^{m: n}(\xi, \zeta) .
$$


$\tilde{F}_{\theta, \boldsymbol{y}}^{m: m}(d x \mid \xi), \tilde{F}_{\theta, \boldsymbol{y}}^{m: n}(d x \mid \xi)$ and $\tilde{G}_{\theta, \boldsymbol{y}}^{m: m}(d x \mid \xi, \zeta), \tilde{G}_{\theta, \boldsymbol{y}}^{m: n}(d x \mid \xi, \zeta)$ are the measures defined by

$$
\begin{aligned}
& \tilde{F}_{\theta, \boldsymbol{y}}^{m: m}(B \mid \xi)=\xi(B), \quad \tilde{F}_{\theta, \boldsymbol{y}}^{m: n}(B \mid \xi)=\int_{B} \tilde{f}_{\theta, \boldsymbol{y}}^{m: n}(x \mid \xi) \mu(d x), \\
& \tilde{G}_{\theta, \boldsymbol{y}}^{m: m}(B \mid \xi, \zeta)=\zeta(B), \quad \tilde{G}_{\theta, \boldsymbol{y}}^{m: n}(B \mid \xi, \zeta)=\int_{B} \tilde{g}_{\theta, \boldsymbol{y}}^{m: n}(x \mid \xi, \zeta) \mu(d x) .
\end{aligned}
$$

Measures $\tilde{F}_{\theta, \boldsymbol{y}}^{m: n}(d x \mid \xi)$ and $\tilde{G}_{\theta, \boldsymbol{y}}^{m: n}(d x \mid \xi, \zeta)$ are also denoted by $\tilde{F}_{\theta, \boldsymbol{y}}^{m: n}(\xi)$ and $\tilde{G}_{\theta, \boldsymbol{y}}^{m: n}(\xi, \zeta)$ (short-hand notation). Then, it is easy to show that $\tilde{F}_{\theta, \boldsymbol{y}}^{m: n}(\xi)$ and $\tilde{G}_{\theta, \boldsymbol{y}}^{m: n}(\xi, \zeta)$ are the optimal filter and its gradient, i.e.,

$$
\tilde{F}_{\theta, \boldsymbol{y}}^{0: n}(B \mid \lambda)=P\left(X_{n}^{\theta, \lambda} \in B \mid Y_{1: n}^{\theta, \lambda}=y_{1: n}\right), \quad \tilde{G}_{\theta, \boldsymbol{y}}^{0: n}(B \mid \lambda, \mathbf{0})=\nabla_{\theta} \tilde{F}_{\theta, \boldsymbol{y}}^{0: n}(B \mid \lambda)
$$

for each $\lambda \in \mathcal{P}(\mathcal{X}), n \geq 1$. Moreover, it is straightforward to verify

$$
\tilde{F}_{\theta, \boldsymbol{y}}^{m: n+1}(\xi)=\tilde{F}_{\theta, y_{n+1}}\left(\tilde{F}_{\theta, \boldsymbol{y}}^{m: n}(\xi)\right), \quad \tilde{G}_{\theta, \boldsymbol{y}}^{m: n+1}(\xi, \zeta)=\tilde{G}_{\theta, y_{n+1}}\left(\tilde{F}_{\theta, \boldsymbol{y}}^{m: n}(\xi), \tilde{G}_{\theta, \boldsymbol{y}}^{m: n}(\xi, \zeta)\right)
$$

for each $\xi \in \mathcal{P}(\mathcal{X}), \zeta \in \mathcal{M}_{s}^{d}(\mathcal{X}), n \geq m \geq 0$.

Remark. We recall here that $Q$ stands for any compact set satisfying $Q \subset \Theta$.

Lemma A2.1. Let Assumptions 2.3 and 2.4 hold. Then, there exists a real number $C_{7, Q} \in[1, \infty)$ (independent of $N$ and depending only on $p_{\theta}\left(x^{\prime} \mid x\right), q_{\theta}(y \mid x)$ ) such that

$$
\begin{aligned}
& \left\|\tilde{F}_{\theta, y}(\xi)-\tilde{F}_{\theta^{\prime}, y}(\xi)\right\| \leq C_{7, Q}\left\|\theta-\theta^{\prime}\right\|, \\
& \left\|\tilde{G}_{\theta, y}(\xi, \zeta)-\tilde{G}_{\theta^{\prime}, y}(\xi, \zeta)\right\| \leq C_{7, Q}\left\|\theta-\theta^{\prime}\right\|(1+\|\zeta\|), \\
& \left\|\tilde{H}_{\theta, y}(\xi, \zeta)-\tilde{H}_{\theta^{\prime}, y}\left(\xi^{\prime}, \zeta^{\prime}\right)\right\| \leq C_{7, Q}\left(\left\|\theta-\theta^{\prime}\right\|+\left\|\xi-\xi^{\prime}\right\|\right)(1+\|\zeta\|)+C_{7, Q}\left\|\zeta-\zeta^{\prime}\right\|
\end{aligned}
$$

for all $\theta, \theta^{\prime} \in Q, \xi, \xi^{\prime} \in \mathcal{P}(\mathcal{X}), \zeta, \zeta^{\prime} \in \mathcal{M}_{s}^{d}(\mathcal{X})$.

Proof. Throughout the proof, the following notation is used. $\theta, \theta^{\prime}$ are any elements of $Q . x, x^{\prime}$ are any elements of $\mathcal{X}$, while $y$ is any element of $\mathcal{Y} . \xi, \xi^{\prime}$ are any elements of $\mathcal{P}(\mathcal{X})$, while $\zeta, \zeta^{\prime}$ are any elements of $\mathcal{M}_{s}^{d}(\mathcal{X})$.

Let $\tilde{C}_{1, Q}=2 \varepsilon_{Q}^{-3} K_{1, Q}(1+\|\mu\|)\left(\varepsilon_{Q}, K_{1, Q}\right.$ are specified in Assumptions 2.3, 2.4, while $\mu(d x)$ is defined in Subsection 2.1). Owing to Assumption 2.3, we have

$$
\varepsilon_{Q}^{2} \leq \varepsilon_{Q} p_{\theta}\left(x^{\prime} \mid x\right) \leq \tilde{r}_{\theta}\left(y, x^{\prime} \mid x\right) \leq \frac{1}{\varepsilon_{Q}} p_{\theta}\left(x^{\prime} \mid x\right) \leq \frac{1}{\varepsilon_{Q}^{2}} .
$$

Consequently, we get

$$
\varepsilon_{Q} \leq \int \tilde{r}_{\theta}\left(y, x^{\prime} \mid x\right) \mu\left(d x^{\prime}\right) \leq \frac{1}{\varepsilon_{Q}}, \quad \varepsilon_{Q}^{3} \leq \tilde{f}_{\theta, y}(x \mid \xi) \leq \frac{1}{\varepsilon_{Q}^{3}} \leq \tilde{C}_{1, Q} .
$$

Moreover, due to Assumptions 2.3, 2.4, we have

$$
\left\|\nabla_{\theta} \tilde{r}_{\theta}\left(x^{\prime} \mid y, x\right)\right\| \leq\left\|\nabla_{\theta} q_{\theta}\left(y \mid x^{\prime}\right)\right\| p_{\theta}\left(x^{\prime} \mid x\right)+q_{\theta}\left(y \mid x^{\prime}\right)\left\|\nabla_{\theta} p_{\theta}\left(x^{\prime} \mid x\right)\right\| \leq \frac{2 K_{1, Q}}{\varepsilon_{Q}} .
$$

Therefore, we get

$$
\left\|\tilde{h}_{\theta, y}(x \mid \xi, \zeta)\right\| \leq \frac{\int\left\|\nabla_{\theta} \tilde{r}_{\theta}\left(y, x \mid x^{\prime}\right)\right\| \xi\left(d x^{\prime}\right)+\int \tilde{r}_{\theta}\left(y, x \mid x^{\prime}\right)|\zeta|\left(d x^{\prime}\right)}{\iint \tilde{r}_{\theta}\left(y, x^{\prime \prime} \mid x^{\prime}\right) \mu\left(d x^{\prime \prime}\right) \xi\left(d x^{\prime}\right)} \leq \frac{2 K_{1, Q}}{\varepsilon_{Q}^{3}}(1+\|\zeta\|) \leq \tilde{C}_{1, Q}(1+\|\zeta\|) .
$$

Hence, we have

$$
\left\|\tilde{H}_{\theta, y}(\xi, \zeta)\right\| \leq \int\left\|\tilde{h}_{\theta, y}(x \mid \xi, \zeta)\right\| \mu(d x) \leq \frac{2 K_{1, Q}\|\mu\|}{\varepsilon_{Q}^{3}}(1+\|\zeta\|) \leq \tilde{C}_{1, Q}(1+\|\zeta\|) .
$$


Let $\tilde{C}_{2, Q}=6 \varepsilon_{Q}^{-3} \tilde{C}_{1, Q} K_{1, Q}^{2}(1+\|\mu\|)$. Due to Assumptions 2.3, 2.4, we have

$$
\begin{aligned}
\left|\tilde{r}_{\theta}\left(x^{\prime} \mid y, x\right)-\tilde{r}_{\theta^{\prime}}\left(x^{\prime} \mid y, x\right)\right| & \leq\left|q_{\theta}\left(y \mid x^{\prime}\right)-q_{\theta^{\prime}}\left(y \mid x^{\prime}\right)\right| p_{\theta}\left(x^{\prime} \mid x\right)+q_{\theta^{\prime}}\left(y \mid x^{\prime}\right)\left|p_{\theta}\left(x^{\prime} \mid x\right)-p_{\theta^{\prime}}\left(x^{\prime} \mid x\right)\right| \\
& \leq \frac{2 K_{1, Q}}{\varepsilon_{Q}}\left\|\theta-\theta^{\prime}\right\| .
\end{aligned}
$$

Owing to Assumptions 2.3, 2.4, we also have

$$
\begin{aligned}
\left\|\nabla_{\theta} \tilde{r}_{\theta}\left(x^{\prime} \mid y, x\right)-\nabla_{\theta} \tilde{r}_{\theta^{\prime}}\left(x^{\prime} \mid y, x\right)\right\| \leq & \left\|\nabla_{\theta} q_{\theta}\left(y \mid x^{\prime}\right)-\nabla_{\theta} q_{\theta^{\prime}}\left(y \mid x^{\prime}\right)\right\| p_{\theta}\left(x^{\prime} \mid x\right) \\
& +\left\|\nabla q_{\theta^{\prime}}\left(y \mid x^{\prime}\right)\right\|\left|p_{\theta}\left(x^{\prime} \mid x\right)-p_{\theta^{\prime}}\left(x^{\prime} \mid x\right)\right| \\
& +\left|q_{\theta}\left(y \mid x^{\prime}\right)-q_{\theta^{\prime}}\left(y \mid x^{\prime}\right)\right|\left\|\nabla_{\theta} p_{\theta}\left(x^{\prime} \mid x\right)\right\| \\
& +q_{\theta^{\prime}}\left(y \mid x^{\prime}\right)\left\|\nabla_{\theta} p_{\theta}\left(x^{\prime} \mid x\right)-\nabla_{\theta} p_{\theta^{\prime}}\left(x^{\prime} \mid x\right)\right\| \\
\leq & \frac{4 K_{1, Q}^{2}}{\varepsilon_{Q}}\left\|\theta-\theta^{\prime}\right\| .
\end{aligned}
$$

Then, using (132), (136), we conclude

$$
\begin{aligned}
\left|\tilde{f}_{\theta, y}(x \mid \xi)-\tilde{f}_{\theta^{\prime}, y}(x \mid \xi)\right| \leq & \frac{\int\left|\tilde{r}_{\theta}\left(y, x \mid x^{\prime}\right)-\tilde{r}_{\theta^{\prime}}\left(y, x \mid x^{\prime}\right)\right| \xi\left(d x^{\prime}\right)}{\iint \tilde{r}_{\theta}\left(y, x^{\prime \prime} \mid x^{\prime}\right) \mu\left(d x^{\prime \prime}\right) \xi\left(d x^{\prime}\right)} \\
& +\tilde{f}_{\theta^{\prime}, y}(x \mid \xi) \frac{\iint\left|\tilde{r}_{\theta}\left(y, x^{\prime \prime} \mid x^{\prime}\right)-\tilde{r}_{\theta^{\prime}}\left(y, x^{\prime \prime} \mid x^{\prime}\right)\right| \mu\left(d x^{\prime \prime}\right) \xi\left(d x^{\prime}\right)}{\iint \tilde{r}_{\theta}\left(y, x^{\prime \prime} \mid x^{\prime}\right) \mu\left(d x^{\prime \prime}\right) \xi\left(d x^{\prime}\right)} \\
\leq & \left(\frac{2 K_{1, Q}}{\varepsilon_{Q}^{2}}+\frac{2 \tilde{C}_{1, Q} K_{1, Q}\|\mu\|}{\varepsilon_{Q}^{2}}\right)\left\|\theta-\theta^{\prime}\right\| \\
& \leq \tilde{C}_{2, Q}\left\|\theta-\theta^{\prime}\right\| .
\end{aligned}
$$

Similarly, relying on (132), (134), (136), (137), we deduce

$$
\begin{aligned}
\left\|\tilde{h}_{\theta, y}(x \mid \xi, \zeta)-\tilde{h}_{\theta^{\prime}, y}(x \mid \xi, \zeta)\right\| \leq & \frac{\int\left|\tilde{r}_{\theta}\left(y, x \mid x^{\prime}\right)-\tilde{r}_{\theta^{\prime}}\left(y, x \mid x^{\prime}\right)\right||\zeta|\left(d x^{\prime}\right)}{\iint \tilde{r}_{\theta}\left(y, x^{\prime \prime} \mid x^{\prime}\right) \mu\left(d x^{\prime \prime}\right) \xi\left(d x^{\prime}\right)} \\
& +\frac{\int\left\|\nabla_{\theta} \tilde{r}_{\theta}\left(y, x \mid x^{\prime}\right)-\nabla_{\theta} \tilde{r}_{\theta^{\prime}}\left(y, x \mid x^{\prime}\right)\right\| \xi\left(d x^{\prime}\right)}{\iint \tilde{r}_{\theta}\left(y, x^{\prime \prime} \mid x^{\prime}\right) \mu\left(d x^{\prime \prime}\right) \xi\left(d x^{\prime}\right)} \\
& +\left\|\tilde{h}_{\theta^{\prime}, y}(x \mid \xi, \zeta)\right\| \frac{\iint\left|\tilde{r}_{\theta}\left(y, x^{\prime \prime} \mid x^{\prime}\right)-\tilde{r}_{\theta^{\prime}}\left(y, x^{\prime \prime} \mid x^{\prime}\right)\right| \mu\left(d x^{\prime \prime}\right) \xi\left(d x^{\prime}\right)}{\iint \tilde{r}_{\theta}\left(y, x^{\prime \prime} \mid x^{\prime}\right) \mu\left(d x^{\prime \prime}\right) \xi\left(d x^{\prime}\right)} \\
& \leq \frac{2 K_{1, Q}}{\varepsilon_{Q}^{2}}\left\|\theta-\theta^{\prime}\right\|\|\zeta\|+\frac{4 K_{1, Q}^{2}}{\varepsilon_{Q}^{2}}\left\|\theta-\theta^{\prime}\right\|+\frac{2 \tilde{C}_{1, Q} K_{1, Q}\|\mu\|}{\varepsilon_{Q}^{2}}\left\|\theta-\theta^{\prime}\right\|(1+\|\zeta\|) \\
\leq & \tilde{C}_{2, Q}\left\|\theta-\theta^{\prime}\right\|(1+\|\zeta\|) .
\end{aligned}
$$

Moreover, (131) - (134) imply

$$
\begin{aligned}
\left\|\tilde{h}_{\theta, y}(\xi, \zeta)-\tilde{h}_{\theta, y}\left(\xi^{\prime}, \zeta^{\prime}\right)\right\| \leq & \frac{\int\left\|\nabla_{\theta} \tilde{r}_{\theta}\left(y, x \mid x^{\prime}\right)\right\|\left|\xi-\xi^{\prime}\right|\left(d x^{\prime}\right)+\int \tilde{r}_{\theta}\left(y, x \mid x^{\prime}\right)\left|\zeta-\zeta^{\prime}\right|\left(d x^{\prime}\right)}{\iint \tilde{r}_{\theta}\left(y, x^{\prime \prime} \mid x^{\prime}\right) \mu\left(d x^{\prime \prime}\right) \xi^{\prime}\left(d x^{\prime}\right)} \\
& +\left\|\tilde{h}_{\theta, y}(\xi, \zeta)\right\| \frac{\iint \tilde{r}_{\theta}\left(y, x^{\prime \prime} \mid x^{\prime}\right) \mu\left(d x^{\prime \prime}\right)\left|\xi-\xi^{\prime}\right|\left(d x^{\prime}\right)}{\iint \tilde{r}_{\theta}\left(y, x^{\prime \prime} \mid x^{\prime}\right) \mu\left(d x^{\prime \prime}\right) \xi^{\prime}\left(d x^{\prime}\right)} \\
& \leq \frac{2 K_{1, Q}}{\varepsilon_{Q}^{2}}\left\|\xi-\xi^{\prime}\right\|+\frac{1}{\varepsilon_{Q}^{3}}\left\|\zeta-\zeta^{\prime}\right\|+\frac{\tilde{C}_{1, Q}}{\varepsilon_{Q}^{3}}\left\|\xi-\xi^{\prime}\right\|(1+\|\zeta\|) \\
& \leq \tilde{C}_{2, Q}\left\|\xi-\xi^{\prime}\right\|(1+\|\zeta\|)+\tilde{C}_{2, Q}\left\|\zeta-\zeta^{\prime}\right\| .
\end{aligned}
$$


Let $\tilde{C}_{3, Q}=2 \tilde{C}_{1, Q} \tilde{C}_{2, Q}(1+\|\mu\|)$. Then, (132), (134), (138), (139) imply

$$
\begin{aligned}
\left\|\tilde{g}_{\theta, y}(x \mid \xi, \zeta)-\tilde{g}_{\theta^{\prime}, y}(x \mid \xi, \zeta)\right\| \leq & \left\|\tilde{h}_{\theta, y}(x \mid \xi, \zeta)-\tilde{h}_{\theta^{\prime}, y}(x \mid \xi, \zeta)\right\| \\
& +\left|\tilde{f}_{\theta, y}(x \mid \xi)-\tilde{f}_{\theta^{\prime}, y}(x \mid \xi)\right| \int\left\|\tilde{h}_{\theta, y}\left(x^{\prime} \mid \xi, \zeta\right)\right\| \mu\left(d x^{\prime}\right) \\
& +\tilde{f}_{\theta^{\prime}, y}(x \mid \xi) \int\left\|\tilde{h}_{\theta, y}\left(x^{\prime} \mid \xi, \zeta\right)-\tilde{h}_{\theta^{\prime}, y}\left(x^{\prime} \mid \xi, \zeta\right)\right\| \mu\left(d x^{\prime}\right) \\
\leq & \left(\tilde{C}_{2, Q}+2 \tilde{C}_{1, Q} \tilde{C}_{2, Q}\|\mu\|\right)\left\|\theta-\theta^{\prime}\right\|(1+\|\zeta\|) \\
\leq & \tilde{C}_{3, Q}\left\|\theta-\theta^{\prime}\right\|(1+\|\zeta\|) .
\end{aligned}
$$

Moreover, (139) yields

$$
\begin{aligned}
\left\|\tilde{H}_{\theta, y}(\xi, \zeta)-\tilde{H}_{\theta^{\prime}, y}(\xi, \zeta)\right\| & \leq \int\left\|\tilde{h}_{\theta, y}(x \mid \xi, \zeta)-\tilde{h}_{\theta^{\prime}, y}(x \mid \xi, \zeta)\right\| \mu(d x) \\
& \leq \tilde{C}_{2, Q}\|\mu\|\left\|\theta-\theta^{\prime}\right\|(1+\|\zeta\|) \\
& \leq \tilde{C}_{3, Q}\left\|\theta-\theta^{\prime}\right\|(1+\|\zeta\|) .
\end{aligned}
$$

Similarly, (140) implies

$$
\begin{aligned}
\left\|\tilde{H}_{\theta, y}(\xi, \zeta)-\tilde{H}_{\theta, y}\left(\xi^{\prime}, \zeta^{\prime}\right)\right\| & \leq \int\left\|\tilde{h}_{\theta, y}(x \mid \xi, \zeta)-\tilde{h}_{\theta, y}\left(x \mid \xi^{\prime}, \zeta^{\prime}\right)\right\| \mu(d x) \\
& \leq \tilde{C}_{2, Q}\|\mu\|\left\|\xi-\xi^{\prime}\right\|(1+\|\zeta\|)+\tilde{C}_{2, Q}\|\mu\|\left\|\zeta-\zeta^{\prime}\right\| \\
& \leq \tilde{C}_{3, Q}\left\|\xi-\xi^{\prime}\right\|(1+\|\zeta\|)+\tilde{C}_{3, Q}\left\|\zeta-\zeta^{\prime}\right\| .
\end{aligned}
$$

Let $C_{7, Q}=\tilde{C}_{3, Q}(1+\|\mu\|)$. Then, using (138), we conclude

$$
\left\|\tilde{F}_{\theta, y}(\xi)-\tilde{F}_{\theta^{\prime}, y}(\xi)\right\| \leq \int\left|\tilde{f}_{\theta, y}(x \mid \xi)-\tilde{f}_{\theta^{\prime}, y}(x \mid \xi)\right| \mu(d x) \leq \tilde{C}_{1, Q}\|\mu\|\left\|\theta-\theta^{\prime}\right\| \leq C_{7, Q}\left\|\theta-\theta^{\prime}\right\|
$$

(notice that $\tilde{C}_{1, Q} \leq \tilde{C}_{3, Q}$ ). Similarly, relying on (141), we deduce

$$
\begin{aligned}
\left\|\tilde{G}_{\theta, y}(\xi, \zeta)-\tilde{G}_{\theta^{\prime}, y}(\xi, \zeta)\right\| & \leq \int\left|\tilde{g}_{\theta, y}(x \mid \xi, \zeta)-\tilde{g}_{\theta^{\prime}, y}(x \mid \xi, \zeta)\right| \mu(d x) \\
& \leq \tilde{C}_{3, Q}\|\mu\|\left\|\theta-\theta^{\prime}\right\|(1+\|\zeta\|) \\
& \leq C_{7, Q}\left\|\theta-\theta^{\prime}\right\|(1+\|\zeta\|) .
\end{aligned}
$$

Moreover, combining (142), (143), we get

$$
\begin{aligned}
\left\|\tilde{H}_{\theta, y}(\xi, \zeta)-\tilde{H}_{\theta^{\prime}, y}\left(\xi^{\prime}, \zeta^{\prime}\right)\right\| & \leq\left\|\tilde{H}_{\theta, y}(\xi, \zeta)-\tilde{H}_{\theta^{\prime}, y}(\xi, \zeta)\right\|+\left\|\tilde{H}_{\theta^{\prime}, y}(\xi, \zeta)-\tilde{H}_{\theta^{\prime}, y}\left(\xi^{\prime}, \zeta^{\prime}\right)\right\| \\
& \leq \tilde{C}_{3, Q}\left(\left\|\theta-\theta^{\prime}\right\|+\left\|\xi-\xi^{\prime}\right\|\right)(1+\|\zeta\|)+\tilde{C}_{3, Q}\left\|\zeta-\zeta^{\prime}\right\| \\
& \leq C_{7, Q}\left(\left\|\theta-\theta^{\prime}\right\|+\left\|\xi-\xi^{\prime}\right\|\right)(1+\|\zeta\|)+C_{7, Q}\left\|\zeta-\zeta^{\prime}\right\| .
\end{aligned}
$$

Lemma A2.2. Let Assumptions 2.3 and 2.4 hold. Then, the following is true:

(i) There exist real numbers $\rho_{4, Q} \in(0,1), C_{8, Q} \in[1, \infty)$ (independent of $N$ and depending only on $\left.p_{\theta}\left(x^{\prime} \mid x\right), q_{\theta}(y \mid x)\right)$ such that

$$
\begin{aligned}
& \left\|\tilde{G}_{\theta, \boldsymbol{y}}^{m: n}(\xi, \zeta)\right\| \leq C_{8, Q}(1+\|\zeta\|), \\
& \left\|\tilde{F}_{\theta, \boldsymbol{y}}^{m: n}(\xi)-\tilde{F}_{\theta, \boldsymbol{y}}^{m: n}\left(\xi^{\prime}\right)\right\| \leq C_{8, Q} \rho_{4, Q}^{n-m}\left\|\xi-\xi^{\prime}\right\|, \\
& \left\|\tilde{G}_{\theta, \boldsymbol{y}}^{m: n}(\xi, \zeta)-\tilde{G}_{\theta, \boldsymbol{y}}^{m: n}\left(\xi^{\prime}, \zeta^{\prime}\right)\right\| \leq C_{8, Q} \rho_{4, Q}^{n-m}\left\|\xi-\xi^{\prime}\right\|(1+\|\zeta\|)+C_{8, Q} \rho_{4, Q}^{n-m}\left\|\zeta-\zeta^{\prime}\right\|
\end{aligned}
$$

for all $\theta \in Q, \xi, \xi^{\prime} \in \mathcal{P}(\mathcal{X}), \zeta, \zeta^{\prime} \in \mathcal{M}_{s}^{d}(\mathcal{X}), n \geq m \geq 0$ and any sequence $\boldsymbol{y}=\left\{y_{n}\right\}_{n \geq 0}$ in $\mathcal{Y}$. 
(ii) There exists a real number $C_{9, Q} \in[1, \infty)$ (independent of $N$ and depending only on $p_{\theta}\left(x^{\prime} \mid x\right), q_{\theta}(y \mid x)$ ) such that

$$
\begin{aligned}
& \left\|\tilde{F}_{\theta: \boldsymbol{y}}^{m: n}(\xi)-\tilde{F}_{\theta^{\prime}, \boldsymbol{y}}^{m: n}(\xi)\right\| \leq C_{9, Q}\left\|\theta-\theta^{\prime}\right\|, \\
& \left\|\tilde{G}_{\theta, \boldsymbol{y}}^{m n}(\xi, \zeta)-\tilde{G}_{\theta^{\prime}, \boldsymbol{y}}^{m: n}(\xi, \zeta)\right\| \leq C_{9, Q}\left\|\theta-\theta^{\prime}\right\|(1+\|\zeta\|)
\end{aligned}
$$

for all $\theta, \theta^{\prime} \in Q, \xi \in \mathcal{P}(\mathcal{X}), \zeta \in \mathcal{M}_{s}^{d}(\mathcal{X}), n \geq m \geq 0$ and any sequence $\boldsymbol{y}=\left\{y_{n}\right\}_{n \geq 0}$ in $\mathcal{Y}$.

Proof. (i) See [29, Theorems 3.1, 3.2] (or [32, Theorem 2.2]).

(ii) Throughout this part of the proof, the following notation is used. $\theta, \theta^{\prime}$ are any elements of $Q . \xi, \zeta$ are any elements of $\mathcal{P}(\mathcal{X}), \mathcal{M}_{s}^{d}(\mathcal{X})$ (respectively). $\boldsymbol{y}=\left\{y_{n}\right\}_{n \geq 0}$ is any sequence in $\mathcal{Y} . n, m$ are any integers satisfying $n>m \geq 0$.

It is straightforward to verify

$$
\begin{aligned}
\tilde{F}_{\theta, \boldsymbol{y}}^{m: n}(\xi)-\tilde{F}_{\theta^{\prime}, \boldsymbol{y}}^{m: n}(\xi) & =\sum_{i=m}^{n-1}\left(\tilde{F}_{\theta, \boldsymbol{y}}^{i: n}\left(\tilde{F}_{\theta^{\prime}, \boldsymbol{y}}^{m: i}(\xi)\right)-\tilde{F}_{\theta, \boldsymbol{y}}^{i+1: n}\left(\tilde{F}_{\theta^{\prime}, \boldsymbol{y}}^{m: i+1}(\xi)\right)\right) \\
& =\sum_{i=m}^{n-1}\left(\tilde{F}_{\theta, \boldsymbol{y}}^{i+1: n}\left(\tilde{F}_{\theta, y_{i}}\left(\tilde{F}_{\theta^{\prime}, \boldsymbol{y}}^{m: i}(\xi)\right)\right)-\tilde{F}_{\theta, \boldsymbol{y}}^{i+1: n}\left(\tilde{F}_{\theta^{\prime}, \boldsymbol{y}}^{m: i+1}(\xi)\right)\right) .
\end{aligned}
$$

It is also easy to show

$$
\begin{aligned}
& \tilde{G}_{\theta, \boldsymbol{y}}^{m: n}(\xi, \zeta)-\tilde{G}_{\theta^{\prime}, \boldsymbol{y}}^{m: n}(\xi, \zeta)= \sum_{i=m}^{n-1}\left(\tilde{G}_{\theta, \boldsymbol{y}}^{i: n}\left(\tilde{F}_{\theta^{\prime}, \boldsymbol{y}}^{m: i}(\xi), \tilde{G}_{\theta^{\prime}, \boldsymbol{y}}^{m: i}(\xi, \zeta)\right)-\tilde{G}_{\theta, \boldsymbol{y}}^{i+1: n}\left(\tilde{F}_{\theta^{\prime}, \boldsymbol{y}}^{m: i+1}(\xi), \tilde{G}_{\theta^{\prime}, \boldsymbol{y}}^{m: i+1}(\xi, \zeta)\right)\right) \\
&=\sum_{i=m}^{n-1}\left(\tilde{G}_{\theta, \boldsymbol{y}}^{i+1: n}\left(\tilde{F}_{\theta, y_{i}}\left(\tilde{F}_{\theta^{\prime}, \boldsymbol{y}}^{m: i}(\xi)\right), \tilde{G}_{\theta, y_{i}}\left(\tilde{F}_{\theta^{\prime}, \boldsymbol{y}}^{m: i}(\xi), \tilde{G}_{\theta^{\prime}, \boldsymbol{y}}^{m: i}(\xi, \zeta)\right)\right)\right. \\
&\left.\quad-\tilde{G}_{\theta, \boldsymbol{y}}^{i+1: n}\left(\tilde{F}_{\theta^{\prime}, \boldsymbol{y}}^{m: i+1}(\xi), \tilde{G}_{\theta^{\prime}, \boldsymbol{y}}^{m: i+1}(\xi, \zeta)\right)\right) .
\end{aligned}
$$

Let $C_{9, Q}=4 C_{7, Q} C_{8, Q}^{2}\left(1-\rho_{4, Q}\right)^{-1}\left(C_{7, Q}\right.$ is specified in Lemma A2.1). Relying on Lemma A2.1 and (145), (147), we deduce

$$
\begin{aligned}
\left\|\tilde{F}_{\theta, \boldsymbol{y}}^{m: n}(\xi)-\tilde{F}_{\theta^{\prime}, \boldsymbol{y}}^{m: n}(\xi)\right\| & \leq \sum_{i=m}^{n-1}\left\|\tilde{F}_{\theta, \boldsymbol{y}}^{i+1: n}\left(\tilde{F}_{\theta, y_{i}}\left(\tilde{F}_{\theta^{\prime}, \boldsymbol{y}}^{m: i}(\xi)\right)\right)-\tilde{F}_{\theta, \boldsymbol{y}}^{i+1: n}\left(\tilde{F}_{\theta^{\prime}, \boldsymbol{y}}^{m: i+1}(\xi)\right)\right\| \\
& \leq C_{8, Q} \sum_{i=m}^{n-1} \rho_{4, Q}^{n-i-1}\left\|\tilde{F}_{\theta, y_{i}}\left(\tilde{F}_{\theta^{\prime}, \boldsymbol{y}}^{m: i}(\xi)\right)-\tilde{F}_{\theta^{\prime}, y_{i}}\left(\tilde{F}_{\theta^{\prime}, \boldsymbol{y}}^{m: i}(\xi)\right)\right\| \\
& \leq C_{7, Q} C_{8, Q}\left\|\theta-\theta^{\prime}\right\| \sum_{i=m}^{n-1} \rho_{4, Q}^{n-i-1} \\
& \leq C_{7, Q} C_{8, Q}\left(1-\rho_{4, Q}\right)^{-1}\left\|\theta-\theta^{\prime}\right\| \leq C_{9, Q}\left\|\theta-\theta^{\prime}\right\| .
\end{aligned}
$$

Similarly, using (146), (148), we conclude

$$
\begin{aligned}
&\left\|\tilde{G}_{\theta, \boldsymbol{y}}^{m: n}(\xi, \zeta)-\tilde{G}_{\theta^{\prime}, \boldsymbol{y}}^{m: n}(\xi, \zeta)\right\| \leq \sum_{i=m}^{n-1} \| \tilde{G}_{\theta, \boldsymbol{y}}^{i+1: n}\left(\tilde{F}_{\theta, y_{i}}\left(\tilde{F}_{\theta^{\prime}, \boldsymbol{y}}^{m: i}(\xi)\right), \tilde{G}_{\theta, y_{i}}\left(\tilde{F}_{\theta^{\prime}, \boldsymbol{y}}^{m: i}(\xi), \tilde{G}_{\theta^{\prime}, \boldsymbol{y}}^{m: i}(\xi, \zeta)\right)\right) \\
& \quad-\tilde{G}_{\theta, \boldsymbol{y}}^{i+1: n}\left(\tilde{F}_{\theta^{\prime}, \boldsymbol{y}}^{m: i+1}(\xi), \tilde{G}_{\theta^{\prime}, \boldsymbol{y}}^{m: i+1}(\xi, \zeta)\right) \| \\
& \leq C_{8, Q} \sum_{i=m}^{n-1} \rho_{4, Q}^{n-i-1}\left\|\tilde{F}_{\theta, y_{i}}\left(\tilde{F}_{\theta^{\prime}, \boldsymbol{y}}^{m: i}(\xi)\right)-\tilde{F}_{\theta^{\prime}, y_{i}}\left(\tilde{F}_{\theta^{\prime}, \boldsymbol{y}}^{m: i}(\xi)\right)\right\|\left(1+\left\|\tilde{G}_{\theta^{\prime}, \boldsymbol{y}}^{m: i+1}(\xi, \zeta)\right\|\right) \\
&+C_{8, Q} \sum_{i=m}^{n-i-1} \rho_{4, Q}^{n-i-1}\left\|\tilde{G}_{\theta, y_{i}}\left(\tilde{F}_{\theta^{\prime}, \boldsymbol{y}}^{m: i}(\xi), \tilde{G}_{\theta^{\prime}, \boldsymbol{y}}^{m: i}(\xi, \zeta)\right)-\tilde{G}_{\theta^{\prime}, y_{i}}\left(\tilde{F}_{\theta^{\prime}, \boldsymbol{y}}^{m: i}(\xi), \tilde{G}_{\theta^{\prime}, \boldsymbol{y}}^{m: i}(\xi, \zeta)\right)\right\| .
\end{aligned}
$$


Consequently, Lemma A2.1 and (144) imply

$$
\begin{aligned}
\left\|\tilde{G}_{\theta, \boldsymbol{y}}^{m: n}(\xi, \zeta)-\tilde{G}_{\theta^{\prime}, \boldsymbol{y}}^{m: n}(\xi, \zeta)\right\| \leq & C_{7, Q} C_{8, Q}\left\|\theta-\theta^{\prime}\right\| \sum_{i=m}^{n-1} \rho_{4, Q}^{n-i-1}\left(1+\left\|\tilde{G}_{\theta^{\prime}, \boldsymbol{y}}^{m: i+1}(\xi, \zeta)\right\|\right) \\
& +C_{7, Q} C_{8, Q}\left\|\theta-\theta^{\prime}\right\| \sum_{i=m}^{n-1} \rho_{4, Q}^{n-i-1}\left(1+\left\|\tilde{G}_{\theta^{\prime}, \boldsymbol{y}}^{m: i}(\xi, \zeta)\right\|\right) \\
\leq & 4 C_{7, Q} C_{8, Q}^{2}\left\|\theta-\theta^{\prime}\right\|(1+\|\zeta\|) \sum_{i=m}^{n-1} \rho_{4, Q}^{n-i-1} \\
\leq & 4 C_{7, Q} C_{8, Q}^{2}\left(1-\rho_{4, Q}\right)^{-1}\left\|\theta-\theta^{\prime}\right\|(1+\|\zeta\|)=C_{9, Q}\left\|\theta-\theta^{\prime}\right\|(1+\|\zeta\|) .
\end{aligned}
$$

Proof of Lemma 4.1. (i) See [32, Theorem 3.1].

(ii) and (iii) Due to $[32$, Proposition 7.2$]$, there exist real numbers $\beta_{Q} \in(0,1), \tilde{C}_{1, Q} \in[1, \infty)$ (independent of $N$ and depending only on $\left.p_{\theta}\left(x^{\prime} \mid x\right), q_{\theta}(y \mid x)\right)$ such that

$$
\left\|E\left(\tilde{H}_{\theta, Y_{n}}\left(\tilde{F}_{\theta, \boldsymbol{Y}}^{0: n-1}(\xi), \tilde{G}_{\theta, \boldsymbol{Y}}^{0: n-1}(\xi, \zeta)\right)-\nabla l(\theta) \mid X_{0}=x, Y_{0}=y\right)\right\| \leq \tilde{C}_{1, Q} \beta_{Q}^{n}(1+\|\zeta\|)
$$

for all $\theta \in Q, x \in \mathcal{X}, y \in \mathcal{Y}, \xi \in \mathcal{P}(\mathcal{X}), \zeta \in \mathcal{M}_{s}^{d}(\mathcal{X}), n \geq 1$.

Throughout these parts of the proof, the following notation is used. $\theta, \theta, \theta^{\prime}$ are any elements of $Q$. $x, y$ are any elements of $\mathcal{X}, \mathcal{Y}$ (respectively), while $B, \xi, \zeta$ are any elements of $\mathcal{B}(\mathcal{X}), \mathcal{P}(\mathcal{X}), \mathcal{M}{ }_{s}^{d}(\mathcal{X})$ (respectively). $\boldsymbol{y}=\left\{y_{n}\right\}_{n \geq 0}$ is any sequence in $\mathcal{Y} . n$ is any positive integer. $\tilde{\alpha}_{\theta, y}(d x \mid \xi), \tilde{\beta}_{\theta, y}(d x \mid \xi, \zeta)$ are the measures defined by

$$
\tilde{\alpha}_{\theta, y}(B \mid \xi)=\frac{\int_{B} q_{\theta}(y \mid x) \xi(d x)}{\int q_{\theta}(y \mid x) \xi(d x)}, \quad \tilde{\beta}_{\theta, y}(B \mid \xi, \zeta)=\frac{\int_{B} q_{\theta}(y \mid x) \zeta(d x)+\int_{B} \nabla_{\theta} q_{\theta}(y \mid x) \xi(d x)}{\int q_{\theta}(y \mid x) \xi(d x)} .
$$

It is straightforward to verify

$$
\begin{aligned}
F_{\theta, \boldsymbol{y}}^{0: n}(B \mid \xi)= & \iint I_{B}\left(x^{\prime}\right) p_{\theta}\left(x^{\prime} \mid x\right) \mu\left(d x^{\prime}\right) \tilde{F}_{\theta, \boldsymbol{y}}^{0: n-1}\left(d x \mid \tilde{\alpha}_{\theta, y_{0}}(\xi)\right), \\
G_{\theta, \boldsymbol{y}}^{0: n}(B \mid \xi, \zeta)= & \iint I_{B}\left(x^{\prime}\right) \nabla_{\theta} p_{\theta}\left(x^{\prime} \mid x\right) \mu\left(d x^{\prime}\right) \tilde{F}_{\theta, \boldsymbol{y}}^{0: n-1}\left(d x \mid \tilde{\alpha}_{\theta, y_{0}}(\xi)\right) \\
& +\iint I_{B}\left(x^{\prime}\right) p_{\theta}\left(x^{\prime} \mid x\right) \mu\left(d x^{\prime}\right) \tilde{G}_{\theta, \boldsymbol{y}}^{0: n-1}\left(d x \mid \tilde{\alpha}_{\theta, y_{0}}(\xi), \tilde{\beta}_{\theta, y_{0}}(\xi, \zeta)\right) .
\end{aligned}
$$

(for a detailed derivation of (150), (151), see [33, Lemma SM2.1]). Then, using (22), (130), we conclude

$$
H_{\theta, y_{n}}\left(F_{\theta, \boldsymbol{y}}^{0: n}(\zeta), G_{\theta, \boldsymbol{y}}^{0: n}(\xi, \zeta)\right)=\tilde{H}_{\theta, y_{n}}\left(\tilde{F}_{\theta, \boldsymbol{y}}^{0: n-1}\left(\tilde{\alpha}_{\theta, y_{0}}(\xi)\right), \tilde{G}_{\theta, \boldsymbol{y}}^{0: n-1}\left(\tilde{\alpha}_{\theta, y_{0}}(\xi), \tilde{\beta}_{\theta, y_{0}}(\xi, \zeta)\right)\right)
$$

Hence, we have

$$
\begin{aligned}
& E\left(H_{\theta, Y_{n}}\left(F_{\theta, \boldsymbol{Y}}^{0: n}(\zeta), G_{\theta, \boldsymbol{Y}}^{0: n}(\xi, \zeta)\right) \mid X_{0}=x, Y_{0}=y\right) \\
& =E\left(\tilde{H}_{\theta, Y_{n}}\left(\tilde{F}_{\theta, \boldsymbol{Y}}^{0: n-1}\left(\tilde{\alpha}_{\theta, y}(\xi)\right), \tilde{G}_{\theta, \boldsymbol{Y}}^{0: n-1}\left(\tilde{\alpha}_{\theta, y}(\xi), \tilde{\beta}_{\theta, y}(\xi, \zeta)\right)\right) \mid X_{0}=x, Y_{0}=y\right) .
\end{aligned}
$$

Let $\tilde{C}_{2, Q}=\varepsilon_{Q}^{-2} K_{1, Q}, \tilde{C}_{3, Q}=5 C_{7, Q} C_{8, Q} C_{9, Q}$, while $C_{1, Q}=3 C_{8, Q} \tilde{C}_{2, Q}(1+\|\mu\|)\left(\varepsilon_{Q}, K_{1, Q}, C_{7, Q}, C_{8, Q}\right.$ are specified in Assumption 2.3, 2.4 and Lemmas A2.1, A2.2, while $\mu(d x)$ is defined in Subsection 2.1). Owing to Assumptions 2.3, 2.4, we have

$$
\left\|\tilde{\beta}_{\theta, y}(\xi, \zeta)\right\| \leq \frac{\int q_{\theta}(y \mid x)|\zeta|(d x)+\int\left\|\nabla_{\theta} q_{\theta}(y \mid x)\right\| \xi(d x)}{\int q_{\theta}(y \mid x) \xi(d x)} \leq \frac{1}{\varepsilon_{Q}^{2}}\|\zeta\|+\frac{K_{1, Q}}{\varepsilon_{Q}} \leq \tilde{C}_{2, Q}(1+\|\zeta\|) .
$$


Consequently, Assumption 2.4, Lemma A2.2 and (151) yield

$$
\begin{aligned}
\left\|G_{\theta, \boldsymbol{y}}^{0: n}(\xi, \zeta)\right\| \leq & \iint\left\|\nabla_{\theta} p_{\theta}\left(x^{\prime} \mid x\right)\right\| \mu\left(d x^{\prime}\right) \tilde{F}_{\theta, \boldsymbol{y}}^{0: n-1}\left(d x \mid \tilde{\alpha}_{\theta, y_{0}}(\xi)\right) \\
& +\iint p_{\theta}\left(x^{\prime} \mid x\right) \mu\left(d x^{\prime}\right)\left|\tilde{G}_{\theta, \boldsymbol{y}}^{0: n-1}\right|\left(d x \mid \tilde{\alpha}_{\theta, y_{0}}(\xi), \tilde{\beta}_{\theta, y_{0}}(\xi, \zeta)\right) \\
\leq & K_{1, Q}\|\mu\|+\left\|\tilde{G}_{\theta, \boldsymbol{y}}^{0: n-1}\left(\tilde{\alpha}_{\theta, y_{0}}(\xi), \tilde{\beta}_{\theta, y_{0}}(\xi, \zeta)\right)\right\| \\
\leq & K_{1, Q}\|\mu\|+C_{8, Q}\left(1+\left\|\tilde{\beta}_{\theta, y_{0}}(\xi, \zeta)\right\|\right) \\
\leq & K_{1, Q}\|\mu\|+2 C_{8, Q} \tilde{C}_{2, Q}(1+\|\zeta\|) \leq C_{1, Q}(1+\|\zeta\|) .
\end{aligned}
$$

Hence, (iii) holds.

Combining (149), (152), (153), we get

$$
\begin{aligned}
\left\|E\left(H_{\theta, Y_{n}}\left(F_{\theta, \boldsymbol{Y}}^{0: n}(\xi), G_{\theta, \boldsymbol{Y}}^{0: n}(\xi, \zeta)\right)-\nabla l(\theta) \mid X_{0}=x, Y_{0}=y\right)\right\| & \leq \tilde{C}_{1, Q} \beta_{Q}^{n}\left(1+\left\|\tilde{\beta}_{\theta, y}(\xi, \zeta)\right\|\right) \\
& \leq 2 \tilde{C}_{1, Q} \tilde{C}_{2, Q} \beta_{Q}^{n}(1+\|\zeta\|) .
\end{aligned}
$$

Therefore, we have

$$
\begin{aligned}
\left\|E\left(H_{\theta, Y_{n}}\left(F_{\theta, \boldsymbol{Y}}^{0: n}(\xi), G_{\theta, \boldsymbol{Y}}^{0: n}(\xi, \zeta)\right)\right)-\nabla l(\theta)\right\| & \leq E\left(\left\|E\left(H_{\theta, Y_{n}}\left(F_{\theta, \boldsymbol{Y}}^{0: n}(\xi), G_{\theta, \boldsymbol{Y}}^{0: n}(\xi, \zeta)\right)-\nabla l(\theta) \mid X_{0}, Y_{0}\right)\right\|\right) \\
& \leq 2 \tilde{C}_{1, Q} \tilde{C}_{2, Q} \beta_{Q}^{n}(1+\|\zeta\|) .
\end{aligned}
$$

Thus, (25) holds.

Owing to Lemmas A2.1, A2.2, we have

$$
\begin{aligned}
& \left\|\tilde{H}_{\theta, Y_{n}}\left(\tilde{F}_{\theta, \boldsymbol{Y}}^{0: n-1}(\xi), \tilde{G}_{\theta, \boldsymbol{Y}}^{0: n-1}(\xi, \zeta)\right)-\tilde{H}_{\theta^{\prime}, Y_{n}}\left(\tilde{F}_{\theta^{\prime}, \boldsymbol{Y}}^{0: n-1}(\xi), \tilde{G}_{\theta^{\prime}, \boldsymbol{Y}}^{0: n-1}(\xi, \zeta)\right)\right\| \\
& \leq C_{7, Q}\left(\left\|\theta-\theta^{\prime}\right\|+\left\|\tilde{F}_{\theta, \boldsymbol{Y}}^{0: n-1}(\xi)-\tilde{F}_{\theta^{\prime}, \boldsymbol{Y}}^{0: n-1}(\xi)\right\|\right)\left(1+\left\|\tilde{G}_{\theta, \boldsymbol{Y}}^{0: n-1}(\xi, \zeta)\right\|\right) \\
& \quad+C_{7, Q}\left\|\tilde{G}_{\theta, \boldsymbol{Y}}^{0: n-1}(\xi, \zeta)-\tilde{G}_{\theta^{\prime}, \boldsymbol{Y}}^{0: n-1}(\xi, \zeta)\right\| \\
& \leq 5 C_{7, Q} C_{8, Q} C_{9, Q}\left\|\theta-\theta^{\prime}\right\|(1+\|\zeta\|) \leq \tilde{C}_{3, Q}\left\|\theta-\theta^{\prime}\right\|(1+\|\zeta\|) .
\end{aligned}
$$

Moreover, due to (149), we have

$$
\begin{aligned}
& \left\|E\left(\tilde{H}_{\theta, Y_{n}}\left(\tilde{F}_{\theta, \boldsymbol{Y}}^{0: n-1}(\xi), \tilde{G}_{\theta, \boldsymbol{Y}}^{0: n-1}(\xi, \zeta)\right)\right)-\nabla l(\theta)\right\| \\
& \leq E\left(\left\|E\left(\tilde{H}_{\theta, Y_{n}}\left(\tilde{F}_{\theta, \boldsymbol{Y}}^{0: n-1}(\xi), \tilde{G}_{\theta, \boldsymbol{Y}}^{0: n-1}(\xi, \zeta)\right)-\nabla l(\theta) \mid X_{0}, Y_{0}\right)\right\|\right) \leq \tilde{C}_{1, Q} \beta_{Q}^{n}(1+\|\zeta\|) .
\end{aligned}
$$

Therefore, we get

$$
\begin{aligned}
\left\|\nabla l(\theta)-\nabla l\left(\theta^{\prime}\right)\right\| \leq & E\left(\left\|\tilde{H}_{\theta, Y_{n}}\left(\tilde{F}_{\theta, \boldsymbol{Y}}^{0: n-1}(\xi), \tilde{G}_{\theta, \boldsymbol{Y}}^{0: n-1}(\xi, \zeta)\right)-\tilde{H}_{\theta^{\prime}, Y_{n}}\left(\tilde{F}_{\theta^{\prime}, \boldsymbol{Y}}^{0: n-1}(\xi), \tilde{G}_{\theta^{\prime}, \boldsymbol{Y}}^{0: n-1}(\xi, \zeta)\right)\right\|\right) \\
& +\left\|E\left(\tilde{H}_{\theta, Y_{n}}\left(\tilde{F}_{\theta, \boldsymbol{Y}}^{0: n-1}(\xi), \tilde{G}_{\theta, \boldsymbol{Y}}^{0: n-1}(\xi, \zeta)\right)\right)-\nabla l(\theta)\right\| \\
& +\left\|E\left(\tilde{H}_{\theta^{\prime}, Y_{n}}\left(\tilde{F}_{\theta^{\prime}, \boldsymbol{Y}}^{0: n-1}(\xi), \tilde{G}_{\theta^{\prime}, \boldsymbol{Y}}^{0: n-1}(\xi, \zeta)\right)\right)-\nabla l\left(\theta^{\prime}\right)\right\| \\
\leq & \tilde{C}_{3, Q}\left\|\theta-\theta^{\prime}\right\|(1+\|\zeta\|)+2 \tilde{C}_{1, Q} \beta_{Q}^{n}(1+\|\zeta\|) .
\end{aligned}
$$

Letting $n \rightarrow \infty$, we deduce

$$
\left\|\nabla l(\theta)-\nabla l\left(\theta^{\prime}\right)\right\| \leq \tilde{C}_{3, Q}\left\|\theta-\theta^{\prime}\right\| .
$$

Since $Q$ is any compact set in $\Theta$, we deduce that (ii) holds. 


\section{References}

[1] M. Benaim, Dynamics of stochastic approximation algorithms, in Lecture Notes in Mathematics, 1709 (1999), pp. $1-68$.

[2] M. Benaim, J. Hofbauer, and S. Sorin, Stochastic approximations and differential inclusions, SIAM Journal on Control and Optimization, 44 (2005), pp. 328 - 348.

[3] A. Benveniste, M. Metivier, and P. Priouret, Adaptive Algorithms and Stochastic Approximations, Springer-Verlag, 1990.

[4] P. J. Bickel, Y. Ritov, and T. Ryden, Asymptotic normality of the maximum likelihood estimator for general hidden Markov models, Annals of Statistics, 26 (1998), pp. 1614 - 1635.

[5] E. Bierstone and P. D. Milman, Semianalytic and subanalytic sets, Institut des Hautes Études Scientifiques, Publications Mathématiques, 67 (1988), pp. 5 - 42 .

[6] V. S. Borkar, Stochastic Approximation: A Dynamical Systems Viewpoint, Cambridge University Press, 2009.

[7] P. Bremaud, Discrete Probability Models and Methods, Springer-Verlag, 2017.

[8] O. Cappé, E. Moulines, and T. Ryden, Inference in Hidden Markov Models, Springer-Verlag, 2005.

[9] D. Crisan and B. Rozovskii, The Oxford Handbook of Nonlinear Filtering, Oxford University Press, 2011.

[10] P. Del Moral, A. Doucet, and S. S. Singh, Uniform stability of a particle approximation of the optimal filter derivative, SIAM Journal on Control and Optimization, 53 (2015), pp 1278 - 1304.

[11] R. Douc and C. Matias, Asymptotics of the maximum likelihood estimator for hidden Markov models for general hidden Markov models, Bernoulli, 7 (2002), pp. 381 - 420 .

[12] R. Douc, E. Moulines, and T. Ryden, Asymptotic properties of the maximum likelihood estimator in autoregressive models with Markov regime, Annals of Statistics, 32 (2004), pp. 2254 - 2304.

[13] R. Douc, G. Fort, E. Moulines and P. Priouret, Forgetting the initial distribution for Hidden Markov Models, Stochastic Processes and their Applications, 119 (2009) pp. 1235-1256.

[14] R. Douc, E. Moulines, and D. S. Stoffer, Nonlinear Time Series: Theory, Methods and Applications with $R$ Examples, CRC Press, 2014.

[15] K. Heine and D. Crisan, Uniform approximations of discrete-time filters, Advances in Applied Probability, 40 (2008), pp. $979-1001$

[16] M. Hurley, Chain recurrence, semiflows, and gradients, Journal of Dynamics and Differential Equations, 7 (1995), pp. $437-435$.

[17] K. Kurdyka, On gradients of functions definable in o-minimal structures, Annales de l'Institut Fourier (Grenoble), 48 (1998), pp. $769-783$.

[18] H. J. Kushner and G. G. Yin, Stochastic Approximation and Recursive Algorithms and Applications, 2nd edition, Springer-Verlag, 2003.

[19] F. Le Gland and L. Mével, Basic properties of the projective product with application to products of column-allowable nonnegative matrices, Mathematics of Control, Signals and Systems 13 (2000), pp $41-62$. 
[20] F. Le Gland and N. Oudjane, Stability and uniform approximation of nonlinear filters using the Hilbert metric and application to particle filters, Annals of Applied Probability, 14 (2004), pp. 144 - 187.

[21] B. G. Leroux, Maximum-likelihood estimation for hidden Markov models, Stochastic Processes and Their Applications, 40 (1992), pp. 127 - 143.

[22] S. P. Meyn and R. L. Tweedie, Markov Chains and Stochastic Stability, 2nd Edition, Cambridge University Press, 2009.

[23] L. Mevel and L. Finesso, Asymptotical statistics of misspecified hidden Markov models, IEEE Transactions on Automatic Control, 49 (2004), pp. 1123 - 1132.

[24] M. K. Pitt and N. Shepard, Filtering via simulation: Auxiliary particle filters, Journal of the American Statistical Association, 94 (1999), pp. 590 - 599.

[25] G. Poyiadjis, A. Doucet, and S. S. Singh, Particle approximations of the score and observed information matrix in state space models with application to parameter estimation, Biometrika 98 (2011), pp. $65-80$.

[26] T. Ryden, Consistent and asymptotically normal parameter estimates for hidden Markov models, Annals of Statistics, 22 (1994), pp. $1884-1895$.

[27] T. Ryden, On recursive estimation for hidden Markov models, Stochastic Processes and Their Applications, 66 (1997), pp. $79-96$.

[28] V. B. Tadić, Analyticity, convergence, and convergence rate of recursive maximum-likelihood estimation in hidden Markov models, IEEE Transactions on Information Theory, 56 (2010), pp. 6406 6432.

[29] V. B. Tadić and A. Doucet, Exponential forgetting and geometric ergodicity for optimal filtering in general state-space models, Stochastic Processes and Their Applications, 115 (2005), pp. 1408-1436.

[30] V. B. Tadić and A. Doucet, Asymptotic bias of stochastic gradient search, Annals of Applied Probability, 27 (2017), pp. 3255-3304.

[31] V. Z. B. Tadić and A. Doucet, Analyticity of entropy rates of continuous-state hidden Markov models, IEEE Transactions on Information Theory, 65 (2019), pp. 7950-7975.

[32] V. Z. B. Tadić and A. Doucet, Stability of optimal filter higher-order derivatives, Stochastic Processes and their Applications 130 (2020), pp. 4808-4858.

[33] V. Z. B. Tadić and A. Doucet, Bias of particle approximations to optimal filter derivative, available at arXiv:1806.09590 (conditionally accepted for publication in SIAM Journal of Control and Optimization).

[34] J. L. Taylor, Several Complex Variables with Connections to Algebraic Geometry and Lie Groups, American Mathematical Society, 2002.

[35] Y. Yomdin, The geometry of critical and near critical values of differentiable mappings, Mathematische Annalen, 264 (1983), pp. 495 - 515. 